Preprint typeset in JHEP style - HYPER VERSION

IFUM-890-FT

UB-ECM-PF-07-05

\title{
Geometry of four-dimensional Killing spinors
}

\author{
Sergio L. Cacciatori, ${ }^{a d}$ Marco M. Caldarelli, ${ }^{b}$ Dietmar Klemm, ${ }^{c d}$ Diego S. Mansi ${ }^{c d}$ \\ and Diederik Roest ${ }^{e}$ \\ a Dipartimento di Scienze Fisiche e Matematiche, \\ Università dell'Insubria, \\ Via Valleggio 11, I-22100 Como. \\ ${ }^{b}$ Departament de Física Fonamental, \\ Universitat de Barcelona, \\ Diagonal, 647, 08028 Barcelona, Spain. \\ ${ }^{c}$ Dipartimento di Fisica dell'Università di Milano, \\ Via Celoria 16, I-20133 Milano. \\ ${ }^{d}$ INFN, Sezione di Milano, Via Celoria 16, I-20133 Milano. \\ e Departament Estructura $i$ Constituents de la Materia, \\ Facultat de Física, Universitat de Barcelona, \\ Diagonal, 647, 08028 Barcelona, Spain. \\ E-mail: sergio.cacciatori@uninsubria.it, caldarelli@ub.edu, \\ dietmar.klemm@mi.infn.it, diego.mansi@mi.infn.it, droest@ecm.ub.es
}

ABstract: The supersymmetric solutions of $\mathcal{N}=2, D=4$ minimal ungauged and gauged supergravity are classified according to the fraction of preserved supersymmetry using spinorial geometry techniques. Subject to a reasonable assumption in the $1 / 2-$ supersymmetric time-like case of the gauged theory, we derive the complete form of all supersymmetric solutions. This includes a number of new $1 / 4$ - and $1 / 2$-supersymmetric possibilities, like gravitational waves on bubbles of nothing in $\mathrm{AdS}_{4}$.

KEYWords: Superstring Vacua, Black Holes, Supergravity Models. 


\section{Contents}

1. Introduction 2

2. $G$-invariant Killing spinors in $4 \mathrm{D}$

2.1 Orbits of Dirac spinors under the gauge group 4

2.2 The ungauged theory 7

2.3 The gauged theory 8

2.4 Generalized holonomy

3. Null representative $1+a e_{1}$

3.1 Constant Killing spinor, $\mathrm{d} a=0$

3.2 Killing spinor with $\mathrm{d} a \neq 0$

3.3 Half-supersymmetric backgrounds

4. Timelike representative $1+b e_{2} \quad \mathbf{1 9}$

4.1 Conditions from the Killing spinor equations

4.2 Geometry of spacetime 20

4.3 Half-supersymmetric backgrounds 25

4.4 Time-dependence of second Killing spinor 28

5. Timelike half-supersymmetric examples 33

5.1 Static Killing spinors and $b=b(z)$

5.1.1 $\mathrm{AdS}_{2} \times \mathbb{H}^{2}$ space-time $(\alpha=0)$

$5.1 .2 \quad \mathrm{AdS}_{4}$ space-time $\left(\beta^{2}=4 \alpha \gamma\right)$

5.1 .3 The Reissner-Nordström-Taub-NUT-AdS 4 family

5.2 Harmonic $b$ solutions 43

5.2.1 Deformations of $\mathrm{AdS}_{2} \times \mathbb{H}^{2}$

5.2 .2 Deformations of $\mathrm{AdS}_{4}$

5.2.3 Deformations of Reissner-Nordström-Taub-NUT-AdS 4

5.3 Imaginary $b$ solutions

5.4 Action of the $\operatorname{PSL}(2, \mathbb{R})$ group on the imaginary $b$ solutions

5.5 Gravitational Chern-Simons system and $G_{0}=\psi_{-}=0$ solutions 50

6. Final remarks

A. Spinors and forms 54 


\section{Introduction}

Throughout the history of string and M-theory an important part in many developments in the subject has been played by supersymmetric solutions of supergravity, i.e. by backgrounds which admit a number of Killing spinors $\epsilon$ which are parallel with respect to the supercovariant derivative ${ }^{1}: D_{\mu} \epsilon=0$. Due to their ubiquitous role it has long been realised that it would be advantageous to have classifications of all supersymmetric solutions of a given theory.

For purely gravitational backgrounds the supersymmetric possibilities follow from the Berger classification of the possible Riemannian holonomies [1] (see [2,3] for an extension to the Lorentzian case). However, in the presence of additional force fields (carried by e. g. scalars, gauge potentials or a cosmological constant) it has proven very difficult to obtain knowledge of all supersymmetric possibilities.

The reason for the complication in the presence of additional fields lies in the holonomy of the supercurvature $R_{\mu \nu}=D_{[\mu} D_{\nu]}$. For purely gravitational backgrounds the holonomy of the supercurvature is generically given by $H=\operatorname{Spin}(d-1,1)$ in $d$ dimensions, and hence coincides with the Lorentz group. In such cases the Lorentz gauge freedom allows one to choose constant Killing spinors. Another simplification is that if there is one Killing spinor with a specific stability subgroup, i.e. it is invariant under some Lorentz subgroup, all other spinors with the same stability subgroup are Killing as well.

For more general solutions including fields other than gravity, the holonomy is generically extended to a larger group $H \supset \operatorname{Spin}(d-1,1)$. For example, in the present paper we consider gauged minimal four-dimensional $\mathcal{N}=2$ supergravity, which has $H=\mathrm{GL}(4, \mathbb{C})[4]$. In such cases one cannot choose constant Killing spinors nor are all spinors with the same stability subgroup automatically Killing. For these reasons the

\footnotetext{
${ }^{1}$ For the purpose of this discussion we will ignore possible additional Killing spinor equations coming from the variation of dilatinos and gauginos.
} 
classification of the backgrounds that allow for Killing spinors is more convoluted, or richer, in such cases. For a long time the only classification available was in ungauged minimal four-dimensional $\mathcal{N}=2$ supergravity [5,6], which has $H=\operatorname{SL}(2, \mathbb{H})$.

A new impulse was given to the subject with the introduction of $G$-structures and the method of spinor bilinears to solve the Killing spinor equations [7]. In this approach, space-time forms are constructed as bilinears from a Killing spinor and one analyses the constraints that these forms imply for the background. Using this framework, a number of complete classifications [8-10] and many partial results (see e.g. [11-21] for an incomplete list) have been obtained. By complete we mean that the most general solutions for all possible fractions of supersymmetry have been obtained, while for partial classifications this is only available for some fractions. Note that the complete classifications mentioned above involve theories with eight supercharges and $H=\mathrm{SL}(2, \mathbb{H})$, and allow for either half- or maximally supersymmetric solutions.

An approach which exploits the linearity of the Killing spinors has been proposed [22] under the name of spinorial geometry. Its basic ingredients are an explicit oscillator basis for the spinors in terms of forms and the use of the gauge symmetry to transform them to a preferred representative of their orbit. In this way one can construct a linear system for the background fields from any (set of) Killing spinor(s) [23]. This method has proven fruitful in e.g. the challenging case of IIB supergravity [24-26]. In addition, it has been adjusted to impose 'near-maximal' supersymmetry and thus has been used to rule out certain large fractions of supersymmetry [27-30]. Finally, a complete classification for type I supergravity in ten dimensions has been obtained [32].

In the present paper we would like to address the classification of supersymmetric solutions in four-dimensional minimal $\mathcal{N}=2$ supergravity. As will also be reviewed in section 2, the ungauged case has been classified completely [5,6]. For the gauged case, the discussion of $1 / 4$ supersymmetry splits up in a time-like and a light-like class (depending on the causal nature of the Killing vector associated to the Killing spinor). The time-like class is completely specified by a single complex function depending on three spatial coordinates $b=b(z, w, \bar{w})$, subject to a second-order differential equation which can not be solved in general [13]. The light-like class can be given in all generality, and in addition its restriction to 1/2-BPS solutions has been derived [16]. Furthermore, there are no backgrounds with $3 / 4$ supersymmetry [29] and $\mathrm{AdS}_{4}$ is the unique possibility with maximal supersymmetry. Therefore the remaining open question concerns half-supersymmetric backgrounds in the gauged theory ${ }^{2}$.

In the following, we will first re-analyse the 1/4-supersymmetric backgrounds using the method of spinorial geometry, and in fact find an additional possibility in the

\footnotetext{
${ }^{2}$ The addition of external matter was considered in [31].
} 
light-like case: a half supersymmetric bubble of nothing in $\mathrm{AdS}_{4}$ and its Petrov type II generalization, a new $1 / 4$ BPS configuration that has the interpretation of gravitational waves propagating on the bubble of nothing. This completes the analysis of the null class in all its generality. Then we will derive the constraints for halfsupersymmetric backgrounds for the timelike class. Subject to a single assumption on the time-dependence of the second Killing spinor these will be solved in general, up to a second order ordinary differential equation. The assumption will be justified by solving the full set of conditions in a number of examples which illustrate the possible spatial dependence of $b$. All these cases turn out to have time-dependence of the assumed form. The different examples are:

- the $b=b(z)$ family of solutions, comprising part of the Reissner-Nordström-TaubNUT-AdS 4 backgrounds,

- waves on the previous backgrounds with $b=b(z, w)$,

- solutions with $b$ imaginary and their $P S L(2, \mathbb{R})$ transformed counterparts,

- solutions of the dimensionally reduced gravitational Chern-Simons model that can be embedded in the equations for a timelike Killing spinor [16].

We determine when these backgrounds preserve $1 / 2$ supersymmetry and provide the explicit Killing spinors. Moreover, in the subcases consisting of $\mathrm{AdS}_{4}$ and $\mathrm{AdS}_{2} \times \mathbb{H}^{2}$, the action of the isometries of these backgrounds on the Killing spinors is given explicitly.

The outline of this paper is as follows. In section 2, we discuss the orbits of Killing spinors and review the known classification results in the theory at hand. In section 3 , we go through the complete classification of the null class. In section 4 , we discuss the constraints for $1 / 4$ and $1 / 2$ supersymmetry in the timelike class. We derive the time-dependence of the second Killing spinor and solve the equations for the case of linear time-dependence $\left(G_{0}=0\right)$. A number of examples of the $1 / 2$ BPS timelike class are provided in section 5 . Finally, in section 6 we present our conclusions and outlook. In appendix A we review our notation and conventions for spinors, while in appendix $\mathrm{B}$ the associated bilinear forms are given. Appendix $\mathrm{C}$ deals with the special case $P^{\prime}=0$, to be defined in section 4.4. Finally, in appendix D, we will give the details of the $G_{0}=0$ case.

\section{G-invariant Killing spinors in $4 \mathrm{D}$}

\subsection{Orbits of Dirac spinors under the gauge group}

In order to obtain the possible orbits of $\operatorname{Spin}(3,1)$ in the space of Dirac spinors $\Delta_{c}$, 
we first consider the most general positive chirality $\operatorname{spinor}^{3} a 1+b e_{12}(a, b \in \mathbb{C})$ and determine its stability subgroup. This is done by solving the infinitesimal equation

$$
\alpha^{c d} \Gamma_{c d}\left(a 1+b e_{12}\right)=0 .
$$

First of all, notice that $a 1+b e_{12}$ is in the same orbit as 1 , which can be seen from

$$
e^{\gamma \Gamma_{13}} e^{\psi \Gamma_{12}} e^{\delta \Gamma_{13}} e^{h \Gamma_{02}} 1=e^{i(\delta+\gamma)} e^{h} \cos \psi 1+e^{i(\delta-\gamma)} e^{h} \sin \psi e_{12} .
$$

This means that we can set $a=1, b=0$ in (2.1), which implies then $\alpha^{02}=\alpha^{13}=0$, $\alpha^{01}=-\alpha^{12}, \alpha^{03}=\alpha^{23}$. The stability subgroup of 1 is thus generated by

$$
X=\Gamma_{01}-\Gamma_{12}, \quad Y=\Gamma_{03}+\Gamma_{23} .
$$

One easily verifies that $X^{2}=Y^{2}=X Y=0$, and thus $\exp (\mu X+\nu Y)=1+\mu X+\nu Y$, so that $X, Y$ generate $\mathbb{R}^{2}$.

Spinors of negative chirality are composed of odd forms, i.e. $a e_{1}+b e_{2}$. One can show in a similar way that they are in the same orbit as $e_{1}$, and the stability subgroup is again $\mathbb{R}^{2}$, with the above generators $X, Y$.

For definiteness and without loss of generality we will always assume that the first Killing spinor has a non-vanishing positive chirality component, and use (part of) the Lorentz symmetry to bring this to the form 1 . Hence we can write a general spinor as $1+a e_{1}+b e_{2}$. Now act with the stability subgroup of 1 to bring $a e_{1}+b e_{2}$ to a special form:

$$
(1+\mu X+\nu Y)\left(1+a e_{1}+b e_{2}\right)=1+b e_{2}+[a+2 b(\nu-i \mu)] e_{1} .
$$

In the case $b=0$ this spinor is invariant, so the representative is $1+a e_{1}$, with isotropy group $\mathbb{R}^{2}$. If $b \neq 0$, one can bring the spinor to the form $1+b e_{2}$, with isotropy group $\mathbb{I}$. The representatives ${ }^{4}$ together with the stability subgroups are summarized in table 1 .

In the ungauged theory, we therefore can have the following $G$-invariant Killing spinors. The $\mathbb{R}^{2}$-invariant Killing spinors are spanned by 1 and $e_{1}$ and there can be up to four of these. The I-invariant Killing spinors are spanned by all four basis elements and there can be up to eight of these. In the first two case, the vector $V_{a}$ bilinear in the spinor $\epsilon$ is lightlike, whereas in the last case it is timelike, see table 1. The existence of a globally defined Killing spinor $\epsilon$, with isotropy group $G \in \operatorname{Spin}(3,1)$, gives rise to a $G$-structure. This means that we have an $\mathbb{R}^{2}$-structure in the null case and an identity structure in the timelike case.

\footnotetext{
${ }^{3}$ Our conventions for spinors and their description in terms of forms can be found in appendix A.

${ }^{4}$ Note the difference in form compared to the Killing spinors of the corresponding theories in five and six dimensions: in six dimensions these can be chosen constant [9] while in five dimensions they are constant up to an overall function [28]. In four dimensions such a choice is generically not possible.
} 
In U(1) gauged supergravity, the local Spin(3,1) invariance is actually enhanced to $\operatorname{Spin}(3,1) \times \mathrm{U}(1)$. Thus, in order to obtain the stability subgroup, one determines the Lorentz transformations that leave a spinor invariant up to an arbitrary phase factor, which can then be gauged away using the additional U(1) symmetry. For the representative 1 , one gets in this way an isotropy group generated by $X, Y$ and $\Gamma_{13}$ obeying

$$
\left[\Gamma_{13}, X\right]=-2 Y, \quad\left[\Gamma_{13}, Y\right]=2 X, \quad[X, Y]=0
$$

i. e. $G \cong \mathrm{U}(1) \ltimes \mathbb{R}^{2}$. For $\epsilon=1+a e_{1}$ with $a \neq 0$, the stability subgroup $\mathbb{R}^{2}$ is not enhanced, whereas the $\mathbb{I}$ of the representative $1+b e_{2}$ is promoted to $\mathrm{U}(1)$ generated by $\Gamma_{13}=i \Gamma_{\text {。. }}$. The Lorentz transformation matrix $a_{A B}$ corresponding to $\Lambda=\exp \left(i \psi \Gamma_{\text {॰. }}\right) \in$ $\mathrm{U}(1)$, with $\Lambda \Gamma_{B} \Lambda^{-1}=a^{A}{ }_{B} \Gamma_{A}$, has nonvanishing components

$$
a_{+-}=a_{-+}=1, \quad a_{\bullet \bullet}=e^{2 i \psi}, \quad a_{\boldsymbol{\bullet \bullet}}=e^{-2 i \psi} .
$$

Finally, notice that in U(1) gauged supergravity one can choose the function $a$ in $1+a e_{1}$ real and positive: Write $a=R \exp (2 i \delta)$, use

$$
e^{\delta \Gamma_{13}}\left(1+a e_{1}\right)=e^{i \delta} 1+e^{-i \delta} a e_{1}=e^{i \delta}\left(1+R e_{1}\right),
$$

and gauge away the phase factor $\exp (i \delta)$ using the electromagnetic $\mathrm{U}(1)$.

\begin{tabular}{||c||c|c||c||}
\hline$\epsilon$ & $G \subset \operatorname{Spin}(3,1)$ & $G \subset \operatorname{Spin}(3,1) \times \mathrm{U}(1)$ & $V_{a}=D\left(\epsilon, \Gamma_{a} \epsilon\right)$ \\
\hline \hline 1 & $\mathbb{R}^{2}$ & $\mathrm{U}(1) \ltimes \mathbb{R}^{2}$ & $(1,0,-1,0)$ \\
\hline $1+a e_{1}$ & $\mathbb{R}^{2}$ & $\mathbb{R}^{2}(a \in \mathbb{R})$ & $\left(1+|a|^{2}, 0,-1-|a|^{2}, 0\right)$ \\
\hline $1+b e_{2}$ & $\mathbb{I}$ & $\mathrm{U}(1)$ & $\left(1+|b|^{2}, 0,-1+|b|^{2}, 0\right)$ \\
\hline
\end{tabular}

Table 1: The representatives $\epsilon$ of the orbits of Dirac spinors and their stability subgroups $G$ under the gauge groups $\operatorname{Spin}(3,1)$ and $\operatorname{Spin}(3,1) \times \mathrm{U}(1)$ in the ungauged and gauged theories, respectively. The number of orbits is the same in both theories, the only difference lies in the stability subgroups and the fact that $a$ is real in the gauged theory. In the last column we give the vectors constructed from the spinors.

In the gauged theory the classification of $G$-invariant spinors is therefore slightly more complicated. There can be at most two $U(1) \ltimes \mathbb{R}^{2}$-invariant Killing spinors, spanned by 1 . The four $\mathbb{R}^{2}$-invariant spinors are spanned by 1 and $e_{1}$. Then there are the $\mathrm{U}(1)$-invariant spinors, spanned by 1 and $e_{2}$. Finally, for generic enough Killing spinors, one does not fall in any of the above classes and the common stability subgroup is $\mathbb{I}$. Note that in the gauged theory the presence of $G$-invariant Killing spinors will in general not lead to a $G$-structure on the manifold but to stronger conditions. The 
structure group is in fact reduced to the intersection of $G$ with $\operatorname{Spin}(3,1)$, and hence is equal to the stability subgroup in the ungauged theory.

We will now consider the possible supersymmetric solutions to the equation $D_{\mu} \epsilon=$ 0 in various sectors of $\mathcal{N}=2, D=4$ in terms of the stability subgroup $G$ of the Killing spinors.

\subsection{The ungauged theory}

The supercovariant derivative of ungauged minimal $\mathcal{N}=2$ supergravity in four dimensions reads

$$
D_{\mu}=\partial_{\mu}+\frac{1}{4} \omega_{\mu}^{a b} \Gamma_{a b}+\frac{i}{4} \mathcal{F}_{a b} \Gamma^{a b} \Gamma_{\mu}
$$

As mentioned in the introduction, a first point to notice is that there is no complex conjugation on the Killing spinor. Therefore, the number of supersymmetries that are preserved is always even: if $\epsilon$ is Killing, then so is $i \epsilon$.

First consider purely gravitational solutions with $\mathcal{F}=0$. In this case the supercovariant connection truncates to the Levi-Civita connection and has $\operatorname{Spin}(3,1)$ holonomy. This implies the following. If $\epsilon$ is Killing, then so $\operatorname{are}^{5} \Gamma_{3} * \epsilon$ and $\Gamma_{012} * \epsilon$ (where $*$ denotes complex conjugation). Together, the operations $i, \Gamma_{3} *$ and $\Gamma_{012} *$ generate four linearly independent Killing spinors from any null spinor $\epsilon=1$ or $\epsilon=1+a e_{1}$ and eight from any time-like spinor $\epsilon=1+b e_{2}$. This illustrates the general statement in the introduction: if the gauge group equals the holonomy, as in this case, then there is only one possible number of Killing spinors for every stability subgroup. Therefore there are only two classes of supersymmetric solutions, which are listed in table 2, and which consist of the gravitational wave and Minkowski space-time, respectively.

\begin{tabular}{||c||c|c||}
\hline$G=\backslash N=$ & 4 & 8 \\
\hline \hline $\mathbb{R}^{2}$ & $\sqrt{ }$ & $\times$ \\
$\mathbb{I}$ & $\times$ & $\sqrt{ }$ \\
\hline
\end{tabular}

Table 2: Gravitational solutions with $G$-invariant Killing spinors in the ungauged theory.

Now let us also allow for fluxes $\mathcal{F}$. The supercovariant connection no longer equals the Levi-Civita connection due to the flux term. In particular, this implies that $\Gamma_{012} *$ no longer commutes with $D_{\mu}$. However, this does still hold for the other operation: $\Gamma_{3} * \epsilon$ is Killing provided $\epsilon$ is. The combined operations of $i$ and $\Gamma_{3} *$ generate four linearly

\footnotetext{
${ }^{5}$ These operations anti-commute and commute with the $\Gamma$-matrices, respectively.
} 
independent spinors from any null or time-like spinor. Thus the number of supersymmetries is always $N=4 p$, as illustrated in table 3. Indeed the generalised holonomy of the supercovariant connection in the ungauged case is $\mathrm{SL}(2, \mathbb{H})[4]$, consistent with the supersymmetries coming in quadruplets.

\begin{tabular}{||c||c|c||}
\hline$G=\backslash N=$ & 4 & 8 \\
\hline \hline $\mathbb{R}^{2}$ & $\sqrt{ }$ & $\times$ \\
$\mathbb{I}$ & $\sqrt{ }$ & $\sqrt{ }$ \\
\hline
\end{tabular}

Table 3: General solutions with $G$-invariant Killing spinors in the ungauged theory.

The half-supersymmetric solution have been classified by Tod [5] and consist of the plane wave and the Israel-Wilson-Perjes metric, respectively. The maximally supersymmetric solutions are $\mathrm{AdS}_{2} \times \mathrm{S}^{2}$ and its Penrose limits, the Hpp wave and Minkowski space-time [6].

\subsection{The gauged theory}

The supercovariant derivative of gauged minimal $\mathcal{N}=2$ supergravity in four dimensions reads

$$
D_{\mu}=\partial_{\mu}+\frac{1}{4} \omega_{\mu}^{a b} \Gamma_{a b}-i \ell^{-1} \mathcal{A}_{\mu}+\frac{1}{2} \ell^{-1} \Gamma_{\mu}+\frac{i}{4} \mathcal{F}_{a b} \Gamma^{a b} \Gamma_{\mu}
$$

Due to the gauging the structure of $\Gamma$-matrices is richer, but there still is no complex conjugation on the Killing spinor. Therefore, the number of supersymmetries that are preserved is always even: if $\epsilon$ is Killing, then so is $i \epsilon$.

Again, we first consider the purely gravitational solutions. In this case the supercovariant derivative has $\mathrm{SO}(3,2)$ holonomy. The operation $\Gamma_{012} *$ commutes with $D_{\mu}$ and therefore generates additional Killing spinors. Together, the operations $i$ and $\Gamma_{012} *$ generate four linearly independent Killing spinors from generic null or time-like spinors. The exception is the null spinor $\epsilon=1+e_{1}$, in which case $\epsilon$ and $\Gamma_{012} *$ are linearly dependent, and hence allows for two instead of four Killing spinors. The possibilities allowed for by this analysis of the supercovariant derivative can be found in table 4 .

However, although all these entries are allowed for by the spinor orbit structure and the crude analysis of the supercurvature above, not all of them have an actual field theoretic realisation in supergravity. In other words, there are no solutions to the Killing spinor equations for all of the above sets of Killing spinors. The lightlike cases were considered in [16]: The 1/4-BPS case is the Lobatchevski wave while imposing more supersymmetries leads to the maximally supersymmetric $\mathrm{AdS}_{4}$ solution (with 


\begin{tabular}{||c||c|c|c|c||}
\hline$G=\backslash N=$ & 2 & 4 & 6 & 8 \\
\hline \hline $\mathrm{U}(1) \ltimes \mathbb{R}^{2}$ & $\times$ & $\times$ & $\times$ & $\times$ \\
$\mathbb{R}^{2}$ & $\sqrt{ }$ & $\circ$ & $\times$ & $\times$ \\
$\mathrm{U}(1)$ & $\times$ & $\circ$ & $\times$ & $\times$ \\
$\mathbb{I}$ & $\times$ & $\circ$ & $\circ$ & $\sqrt{ }$ \\
\hline
\end{tabular}

Table 4: Gravitational solutions with $G$-invariant Killing spinors in the gauged theory. Check marks indicate entries with actual solutions, while circles stand for allowed entries which are not realized.

$G=1)$. The $N=4$ and $G=\mathbb{R}^{2}$ entry is thus effectively empty. In particular, this implies that imposing a single Killing spinor $1+a e_{1}$ with $a \neq 1$ leads to $\mathrm{AdS}_{4}$. Also note that the $N=6$ and $G=1$ entry must be empty since any time-like spinor plus $1+e_{1}$ leads to maximal supersymmetry, while all other Killing spinors come in groups of four. The only remaining entries are $N=4$ and $G=\mathrm{U}(1)$ or $G=\mathbb{I}$. Using the results of $[13,16]$, it is straightforward to show that in these purely gravitational timelike cases the geometry is given by

$$
d s^{2}=-\frac{z^{2}+n^{2}}{\ell^{2}}(d t-2 n \cosh \theta d \phi)^{2}+\frac{\ell^{2} d z^{2}}{z^{2}+n^{2}}+\left(z^{2}+n^{2}\right)\left(d \theta^{2}+\sinh ^{2} \theta d \phi^{2}\right),
$$

where $n= \pm \ell / 2$. But this is simply $\mathrm{AdS}_{4}$ written as a line bundle over a threedimensional base manifold, so both $N=4$ entries are empty as well. We conclude that there are no 1/2-supersymmetric gravitational solutions in the gauged theory, only the 1/4-supersymmetric Lobatchevski waves and maximally supersymmetric $\mathrm{AdS}_{4}$.

We now come to the general supersymmetric solutions in the gauged case. Due to the gauging and flux terms, neither $\Gamma_{012} *$ nor $\Gamma_{3} *$ commute with $D_{\mu}$. Therefore we have the cases as listed in table 5. The supercovariant connection in the gauged case has generalized holonomy $\mathrm{GL}(4, \mathbb{C})[4]$, again consistent with the supersymmetries coming in doublets.

The $1 / 4$-BPS solutions with $G=\mathbb{R}^{2}$ and $G=U(1)$ were derived in [13], and we will show there is no solution with $G=\mathrm{U}(1) \ltimes \mathbb{R}^{2}$. In addition, it was shown in [16] that any additional supersymmetries in the null case are always timelike, i.e. end up in the $N=4$ and $G=1$ entry. Again, the $N=4$ and $G=\mathbb{R}^{2}$ entry is empty. It would be interesting to see if there is a nice explanation for this. In addition, the maximally supersymmetric case is always $\mathrm{AdS}_{4}$. Recently, it has been shown in [29] that the $N=6$ and $G=1$ entry is empty as well, because imposing three complex Killing spinors implies that the spacetime is $\mathrm{AdS}_{4}$ and thus maximally supersymmetric. 


\begin{tabular}{||c||c|c|c|c||}
\hline$G=\backslash N=$ & 2 & 4 & 6 & 8 \\
\hline \hline $\mathrm{U}(1) \ltimes \mathbb{R}^{2}$ & $\circ$ & $\times$ & $\times$ & $\times$ \\
$\mathbb{R}^{2}$ & $\sqrt{ }$ & $\circ$ & $\times$ & $\times$ \\
$\mathrm{U}(1)$ & $\sqrt{ }$ & $\sqrt{ }$ & $\times$ & $\times$ \\
$\mathbb{I}$ & $\times$ & $\sqrt{ }$ & $\circ$ & $\sqrt{ }$ \\
\hline
\end{tabular}

Table 5: General solutions with $G$-invariant Killing spinors in the gauged theory. Check marks indicate entries with actual solutions, while circles stand for allowed entries which are not realized.

The most general 1/2-BPS solution in the timelike case remains an open issue and will be studied in this paper.

\subsection{Generalized holonomy}

In minimal gauged supergravity theories with eight supercharges, the generalized holonomy group for vacua preserving $N$ supersymmetries, where $N=0,2,4,6,8$, is $\operatorname{GL}\left(\frac{8-N}{2}, \mathbb{C}\right)$ $\ltimes \frac{N}{2} \mathbb{C}^{\frac{8-N}{2}}[4]$. To see this, assume that there exists a Killing spinor $\epsilon_{1}$. By a local $\operatorname{GL}(4, \mathbb{C})$ transformation, $\epsilon_{1}$ can be brought to the form $\epsilon_{1}=(1,0,0,0)^{T}$. This is annihilated by matrices of the form

$$
\mathcal{A}=\left(\begin{array}{ll}
0 & \underline{a}^{T} \\
\underline{0} & A
\end{array}\right)
$$

that generate the affine group $\mathrm{A}(3, \mathbb{C}) \cong \mathrm{GL}(3, \mathbb{C}) \ltimes \mathbb{C}^{3}$. Now impose a second Killing spinor $\epsilon_{2}=\left(\epsilon_{2}^{0}, \underline{\epsilon}_{2}\right)^{T}$. Acting with the stability subgroup of $\epsilon_{1}$ yields

$$
e^{\mathcal{A}} \epsilon_{2}=\left(\begin{array}{c}
\epsilon_{2}^{0}+\underline{b}^{T} \underline{\epsilon}_{2} \\
e^{A} \underline{\epsilon}_{2}
\end{array}\right), \quad \text { where } \quad \underline{b}^{T}=\underline{a}^{T} A^{-1}\left(e^{A}-1\right) .
$$

We can choose $A \in \operatorname{gl}(3, \mathbb{C})$ such that $e^{A} \underline{\epsilon}_{2}=(1,0,0)^{T}$, and $\underline{b}$ such that $\epsilon_{2}^{0}+\underline{b}^{T} \underline{\epsilon}_{2}=0$. This means that the stability subgroup of $\epsilon_{1}$ can be used to bring $\epsilon_{2}$ to the form $\epsilon_{2}=(0,1,0,0)$. The subgroup of $\mathrm{A}(3, \mathbb{C})$ that stabilizes also $\epsilon_{2}$ consists of the matrices

$$
\left(\begin{array}{cccc}
1 & 0 & b_{2} & b_{3} \\
0 & 1 & B_{12} & B_{13} \\
0 & 0 & B_{22} & B_{23} \\
0 & 0 & B_{32} & B_{33}
\end{array}\right) \quad \in \mathrm{GL}(2, \mathbb{C}) \ltimes 2 \mathbb{C}^{2} .
$$

Finally, imposing a third Killing spinor yields $\mathrm{GL}(1, \mathbb{C}) \ltimes 3 \mathbb{C}$ as maximal generalized holonomy group, which is however not realized in $\mathcal{N}=2, D=4$ minimal gauged 
supergravity $[16,29]$. It would be interesting to better understand why such preons actually do not exist. In section 4.3, we explicitely compute the generalized holonomy group for $\mathcal{N}=2, D=4$ minimal gauged supergravity in the case $N=2$ and show that it is indeed contained in $\mathrm{A}(3, \mathbb{C})$, supporting thus the classification scheme of [4].

\section{Null representative $1+a e_{1}$}

In this section we will analyse the conditions coming from a single null Killing spinor. As we saw in section 2.1, there are two orbits of such spinors, one with representative $\epsilon=1$ and stability subgroup $G=\mathrm{U}(1) \ltimes \mathbb{R}^{2}$ and one with $\epsilon=1+a e_{1}$ and $G=\mathbb{R}^{2}$. Owing to local $\mathrm{U}(1)$ gauge invariance, it is always possible to choose the function $a$ real and positive, so in the following we set $a=e^{\chi}, \chi \in \mathbb{R}$. The Killing spinor equations become

$$
\begin{aligned}
-\frac{i}{\ell} \mathcal{A}+\frac{1}{2} \Omega+\frac{e^{\chi}}{\sqrt{2}}\left[\left(\frac{1}{\ell}+i \phi\right) E^{\overline{\boldsymbol{\bullet}}}-2 i \mathcal{F}^{+\overline{\boldsymbol{}}} E^{-}\right] & =0, \\
\mathrm{~d} \chi+\frac{i}{\ell} \mathcal{A}+\frac{1}{2} \Omega+\frac{e^{-\chi}}{\sqrt{2}}\left[\left(\frac{1}{\ell}-i \phi\right) E^{\overline{\boldsymbol{\bullet}}}+2 i \mathcal{F}^{+\boldsymbol{\bullet}} E^{-}\right] & =0, \\
\omega^{-\bullet}+\frac{e^{\chi}}{\sqrt{2}}\left[2 i \mathcal{F}^{-\bullet} E^{\overline{\boldsymbol{\bullet}}}+\left(\frac{1}{\ell}-i \phi\right) E^{-}\right] & =0, \\
\omega^{-\bullet}+\frac{e^{-\chi}}{\sqrt{2}}\left[-2 i \mathcal{F}^{-\bullet} E^{\overline{\boldsymbol{\bullet}}}+\left(\frac{1}{\ell}+i \phi\right) E^{-}\right] & =0,
\end{aligned}
$$

where $\phi \equiv \mathcal{F}^{+-}+\mathcal{F}^{\bar{\bullet} \bullet}$ and $\Omega \equiv \omega^{+-}+\omega^{\bar{\bullet} \bullet}$.

The conditions for the special $\mathrm{U}(1) \ltimes \mathbb{R}^{2}$-orbit with $\epsilon=1$ can be obtained as the singular limit $\chi \rightarrow-\infty$ of the above equations. Note however that, in this limit, the second line implies the constraint $\ell^{-1}-i \phi=0$, while the fourth line leads to $\ell^{-1}+i \phi=0$. Clearly, for $\ell^{-1} \neq 0$ this does not allow for a solution. Hence, in the gauged theory, there are no backgrounds with $\mathrm{U}(1) \ltimes \mathbb{R}^{2}$-invariant Killing spinors.

The only null possibility is therefore given by the $\mathbb{R}^{2}$-invariant Killing spinor $\epsilon=$ $1+e^{\chi} e_{1}$. We will now analyse the above conditions for the generic case with $\chi$ finite. In fact, we will furthermore assume it is positive. This does not constitute any loss of generality since one can flip the sign of $\chi$ by changing chirality (a spinor $1+e^{\chi} e_{1}$ with $\chi$ negative is gauge equivalent to a spinor $e_{1}+e^{\tilde{\chi}} 1$ with $\tilde{\chi}=-\chi$ positive), and hence the resulting background will not depend on this sign.

From the last two equations one obtains the constraints

$$
\mathcal{F}^{-\bullet}=\mathcal{F}^{-\bar{\bullet}}=0, \quad \phi=-\frac{i}{\ell} \tanh \chi
$$


on the field strength, as well as

$$
\omega^{-\bullet}=\omega^{-\bullet}=-\frac{1}{\sqrt{2} \ell \cosh \chi} E^{-}
$$

for the spin connection. (3.2) implies $\mathcal{F}^{+-}=0$ and $\mathcal{F}^{\boldsymbol{\bullet} \bullet}=-\frac{i}{\ell} \tanh \chi$. The first two equations of (3.1) yield then

$$
\begin{aligned}
\omega^{+-} & =2 e^{\chi} H_{3} E^{-}-\frac{1}{\ell} \frac{e^{2 \chi}}{\cosh \chi} E^{1}, \\
\omega^{\mathbf{\bullet} \bullet} & =2 i \sinh \chi H_{1} E^{-}+\frac{i}{\ell} \frac{\cosh 2 \chi}{\cosh \chi} E^{3}, \\
\mathcal{A} & =-\ell \cosh \chi H_{1} E^{-}-\sinh \chi E^{3}, \\
\mathrm{~d} \chi & =-2 \cosh \chi H_{3} E^{-}+\frac{2}{\ell} \sinh \chi E^{1},
\end{aligned}
$$

where $E^{1}=\left(E^{\bullet}+E^{\bar{\bullet}}\right) / \sqrt{2}, i E^{3}=\left(E^{\bullet}-E^{\bar{\bullet}}\right) / \sqrt{2}$, and we defined

$$
\frac{\mathcal{F}^{+\bullet}+\mathcal{F}^{+\boldsymbol{\bullet}}}{\sqrt{2}}=H_{1}, \quad \frac{\mathcal{F}^{+\bullet}-\mathcal{F}^{+\boldsymbol{\bullet}}}{\sqrt{2}}=i H_{3} .
$$

In order to proceed, we distinguish two subcases, namely $\mathrm{d} \chi=0$ and $\mathrm{d} \chi \neq 0$.

\subsection{Constant Killing spinor, $\mathrm{d} a=0$}

If $a$ and hence $\chi$ are constant, eqn. (3.4) implies $\chi=H_{3}=0$. Next we impose vanishing torsion. The torsion two-form reads

$$
\begin{aligned}
& T^{-}=\mathrm{d} E^{-}+\frac{2}{\ell} E^{1} \wedge E^{-}, \\
& T^{+}=\mathrm{d} E^{+}-E^{1} \wedge\left(\omega^{+1}+\frac{E^{+}}{\ell}\right)+\omega^{+3} \wedge E^{3}, \\
& T^{1}=\mathrm{d} E^{1}+E^{-} \wedge\left(\omega^{+1}+\frac{E^{+}}{\ell}\right), \\
& T^{3}=\mathrm{d} E^{3}+\frac{1}{\ell} E^{1} \wedge E^{3}-\omega^{+3} \wedge E^{-} .
\end{aligned}
$$

From $T^{-}=0$ one gets $E^{-} \wedge \mathrm{d} E^{-}=0$, so by Fröbenius' theorem there exist two functions $\eta$ and $u$ such that locally

$$
E^{-}=\eta \mathrm{d} u
$$

Plugging this into $T^{-}=0$ yields

$$
\eta\left(\mathrm{d} \log \eta+\frac{2}{\ell} E^{1}\right) \wedge \mathrm{d} u=0,
$$


so that there exists a function $\xi$ such that

$$
E^{1}=-\frac{\ell}{2 \eta} \mathrm{d} \eta+\xi \mathrm{d} u
$$

The gauge field and its field strength can now be written as

$$
\mathcal{A}=-\ell \eta H_{1} \mathrm{~d} u, \quad \mathcal{F}=\frac{\ell}{2} H_{1} \mathrm{~d} \eta \wedge \mathrm{d} u,
$$

and the Bianchi identity $\mathcal{F}=\mathrm{d} \mathcal{A}$ implies

$$
\left(\mathrm{d} H_{1}+\frac{3}{2} H_{1} \mathrm{~d} \log \eta\right) \wedge \mathrm{d} u=0 .
$$

This means that $H_{1} \eta^{3 / 2}$ can depend only on $u$,

$$
H_{1} \eta^{3 / 2}=-\frac{\varphi^{\prime}(u)}{\ell}
$$

where the prefactor and the derivative were chosen in order to conform with the notation of [13]. Let us define a new coordinate $x=-\eta^{-1 / 2}$, so that $E^{1}=\frac{\ell}{x} \mathrm{~d} x+\xi \mathrm{d} u, E^{-}=x^{-2} \mathrm{~d} u$ and

$$
\mathcal{A}=-x \varphi^{\prime}(u) \mathrm{d} u .
$$

One can now use part of the residual gauge freedom, given by the stability subgroup $\mathbb{R}^{2}$ of the null spinor $1+a e_{1}$, in order to simplify $E^{1}$. To this end, consider an $\mathbb{R}^{2}$ transformation with group element

$$
\Lambda=1+\mu X+\nu Y,
$$

where $X$ and $Y$ are given in (2.2). Defining $\alpha=\mu+i \nu$, this can also be written as

$$
\Lambda=1+\alpha \Gamma_{+\bullet}+\bar{\alpha} \Gamma_{+} \cdot
$$

Given the ordering $A, B=+,-, \bullet, \bar{\bullet}$, the Lorentz transformation matrix $a_{A B}$ corresponding to $\Lambda \in \mathbb{R}^{2} \subseteq \operatorname{Spin}(3,1)$ reads

$$
a_{A B}=\left(\begin{array}{cccc}
0 & 1 & 0 & 0 \\
1 & -4|\alpha|^{2} & 2 \bar{\alpha} & 2 \alpha \\
0 & -2 \bar{\alpha} & 0 & 1 \\
0 & -2 \alpha & 1 & 0
\end{array}\right) .
$$

The transformed vielbein ${ }^{\alpha} E^{A}=a^{A}{ }_{B} E^{B}$ is thus given by

$$
\begin{aligned}
{ }^{\alpha} E^{\bullet} & =E^{\bullet}-2 \alpha E^{-}, & & { }^{\alpha} E^{1}=E^{1}-\sqrt{2}(\alpha+\bar{\alpha}) E^{-}, \\
{ }^{\alpha} E^{\bar{\bullet}} & =E^{\bar{\bullet}}-2 \bar{\alpha} E^{-}, & & { }^{\alpha} E^{3}=E^{3}+\sqrt{2} i(\alpha-\bar{\alpha}) E^{-}, \\
{ }^{\alpha} E^{-} & =E^{-}, & & { }^{\alpha} E^{+}=E^{+}+2 \bar{\alpha} E^{\bullet}+2 \alpha E^{\mathbf{\bullet}}-4|\alpha|^{2} E^{-} .
\end{aligned}
$$


Choosing $\alpha+\bar{\alpha}=\xi x^{2} / \sqrt{2}$, we can eliminate $E_{u}^{1}$, so one can set $\xi=0$ without loss of generality. Note that this still leaves a residual gauge freedom associated to the imaginary part of $\alpha$, which will be used below.

From $\mathrm{d} T^{3}=0$ we get $\mathrm{d}\left(\omega^{+3} / x\right) \wedge \mathrm{d} u=0$, and thus there exist two functions $\beta, \tilde{\beta}$ such that

$$
\omega^{+3}=-x \mathrm{~d} \beta+\tilde{\beta} \mathrm{d} u
$$

Plugging this into $T^{3}=0$ yields $\mathrm{d}\left(x E^{3}+\beta \mathrm{d} u\right)=0$, which is solved by

$$
E^{3}=-\frac{\ell}{x} \mathrm{~d} y+\beta \mathrm{d} u
$$

where $y$ denotes some function that we shall use as a coordinate. Using the remaining gauge freedom (3.8) with $\operatorname{Im} \alpha=-\beta x^{2} / 2 \sqrt{2}$ allows to set also $\beta=0$. The equation $T^{1}=0$ tells us that $\omega^{+1}+E^{+} / \ell=\gamma \mathrm{d} u$ for some function $\gamma$. Using this together with $T^{+}=0$, one shows that

$$
\mathrm{d}\left(E^{-} \wedge E^{+}\right)=-\frac{2}{x} \mathrm{~d} x \wedge\left(E^{-} \wedge E^{+}\right)
$$

which means that the surface described by $E^{-}$and $E^{+}$is integrable, so that

$$
E^{+}=\ell^{2} \frac{\mathcal{G}}{2} \mathrm{~d} u+h \mathrm{~d} V
$$

for some functions $\mathcal{G}, h, V$. The metric becomes then

$$
\begin{aligned}
\mathrm{d} s^{2} & =2 E^{-} E^{+}+\left(E^{1}\right)^{2}+\left(E^{3}\right)^{2} \\
& =\frac{\ell^{2}}{x^{2}}\left(\mathcal{G} \mathrm{d} u^{2}+\frac{2 h}{\ell^{2}} \mathrm{~d} u \mathrm{~d} V+\mathrm{d} x^{2}+\mathrm{d} y^{2}\right) .
\end{aligned}
$$

Finally, the equation $T^{+}=0$ implies

$$
\begin{aligned}
\partial_{x} h & =\partial_{y} h=0, \quad \partial_{V} \mathcal{G}=\frac{2}{\ell^{2}} \partial_{u} h, \\
\gamma & =\frac{x \ell}{2} \partial_{x} \mathcal{G}, \quad \tilde{\beta}=-\frac{x \ell}{2} \partial_{y} \mathcal{G} .
\end{aligned}
$$

$h$ can be eliminated by introducing a new coordinate $v(u, V)$ with $\partial_{V} v=h / \ell^{2}$ and shifting $\mathcal{G} \rightarrow \mathcal{G}+2 \partial_{u} v$, which leads to

$$
\mathrm{d} s^{2}=\frac{\ell^{2}}{x^{2}}\left(\mathcal{G} \mathrm{d} u^{2}+2 \mathrm{~d} u \mathrm{~d} v+\mathrm{d} x^{2}+\mathrm{d} y^{2}\right) .
$$

Note that, due to $(3.13), \mathcal{G}$ is independent of $v$, therefore $\partial_{v}$ is a Killing vector. One easily verifies that it coincides with the Killing vector constructed from the Killing spinor as $-\frac{\ell^{2}}{2 \sqrt{2}} D\left(\epsilon, \Gamma_{\mu} \epsilon\right)$. 
All that remains is to impose the Maxwell and Einstein equations. One finds that the former are automatically satisfied by the gauge potential (3.6). The same holds for the Einstein equations, except for the uu-component, which gives the Siklos equation with sources

$$
\Delta \mathcal{G}-\frac{2}{x} \partial_{x} \mathcal{G}=-\frac{4 x^{2}}{\ell^{2}} \varphi^{\prime}(u)^{2}
$$

This family of solutions enjoys a large group of diffeomorphisms which leave the solution invariant in form but change the function $\mathcal{G}$. This is the Siklos-Virasoro invariance, discussed in $[16,33]$. In conclusion, the geometry of solutions admitting the constant null spinor $1+e_{1}$ is given by the Lobachevski waves with metric (3.14) and gauge field (3.6), where $\mathcal{G}$ satisfies (3.15) and $\varphi(u)$ is arbitrary. This coincides exactly with the results of [13], where it was shown moreover that there is a second covariantly constant spinor iff the wave profiles $\mathcal{G}$ and $\varphi$ have the form

$$
\mathcal{G}_{\alpha}(x, y, u)=-\frac{x^{4}}{\ell^{2}}+2 \alpha x^{3}-\alpha^{2} \ell^{2}\left(x^{2}+y^{2}\right), \quad \varphi(u)=u
$$

up to Siklos-Virasoro transformation, with $\alpha \in \mathbb{R}$ constant. In this case, the solution does also belong to the timelike class [13]. While the $\alpha \neq 0$ solution only has the obvious Killing vectors $\partial_{v}$ and $\partial_{y}$, the special $\alpha=0$ case is maximally symmetric with a five-dimensional isometry group.

\subsection{Killing spinor with $\mathrm{d} a \neq 0$}

If $\mathrm{d} a$ and hence also $\mathrm{d} \chi$ do not vanish, one can use the $\mathbb{R}^{2}$ stability subgroup of the spinor $1+e^{\chi} e_{1}$ to eliminate the fluxes $\mathcal{F}^{+\bullet}$ and $\mathcal{F}^{+\boldsymbol{\bullet}}$. To see this, observe that under an $\mathbb{R}^{2}$ transformation (3.8),

$$
{ }^{\alpha} \mathcal{F}^{+\bullet}=\mathcal{F}^{+\bullet}-\frac{2 i \alpha}{\ell} \tanh \chi, \quad{ }^{\alpha} \mathcal{F}^{\bar{\bullet} \bullet}=\mathcal{F}^{\bar{\bullet} \bullet}
$$

so by choosing $\alpha=-\frac{i \ell}{2} \mathcal{F}^{+\bullet} \operatorname{coth} \chi$ one can achieve ${ }^{\alpha} \mathcal{F}^{+\bullet}=0$. Note that this would not be possible if $\chi=0$. With this gauge fixing, one has

$$
\mathrm{d} \chi=\frac{2}{\ell} \sinh \chi E^{1}, \quad \mathcal{A}=-\sinh \chi E^{3}, \quad \mathcal{F}=-\frac{1}{\ell} \tanh \chi E^{1} \wedge E^{3} .
$$

Next we impose vanishing torsion. Using (3.17), one easily shows that $T^{-}=0$ leads to

$$
\mathrm{d}\left[\left(e^{2 \chi}-1\right) E^{-}\right]=0,
$$

and therefore one can introduce a function $u$ with

$$
\left(e^{2 \chi}-1\right) E^{-}=\mathrm{d} u .
$$


Before we come to the other torsion components, let us consider the Bianchi identity and the Maxwell equations. The gauge field strength reads

$$
\mathcal{F}=\frac{\mathrm{d} \chi}{\sinh 2 \chi} \wedge \mathcal{A}
$$

Requiring it to be equal to $\mathrm{d} \mathcal{A}$ implies that $\mathcal{A} / \sqrt{\tanh \chi}$ is closed, so that locally

$$
\mathcal{A}=\sqrt{\tanh \chi} \mathrm{d} \Psi
$$

Note that the functions $\chi, u$ and $\Psi$ must be independent, because otherwise $E^{1}, E^{-}$ and $E^{3}$ would not be linearly independent. We can thus use these three functions as coordinates.

Using

$$
* \mathcal{F}=-\frac{1}{\ell} \tanh \chi E^{-} \wedge E^{+},
$$

the Maxwell equations $\mathrm{d}^{*} \mathcal{F}=0$ imply

$$
\mathrm{d}\left(E^{-} \wedge E^{+}\right)+2 \frac{\mathrm{d} \chi}{\sinh 2 \chi} \wedge\left(E^{-} \wedge E^{+}\right)=0 .
$$

By Fröbenius' theorem and (3.18), $E^{+}$can thus be written as

$$
E^{+}=\frac{\tilde{\mathcal{K}}}{2} \mathrm{~d} u+h \mathrm{~d} V,
$$

where $\tilde{\mathcal{K}}, h$ and $V$ are some functions, and we can use $V$ as the remaining coordinate. Substituing $E^{+}$into the Maxwell equations one obtains a constraint on the function $h$,

$$
\mathrm{d}\left(\frac{h}{e^{2 \chi}+1}\right) \wedge \mathrm{d} u \wedge \mathrm{d} V=0
$$

and hence

$$
h=h_{0}(u, V)\left(e^{2 \chi}+1\right) .
$$

In what follows, we define $\mathcal{K}=\tilde{\mathcal{K}} /\left(e^{2 \chi}+1\right)$ and use $\omega^{+1}=\left(\omega^{+\bullet}+\omega^{+\bar{\bullet}}\right) / \sqrt{2}, \omega^{+3}=$ $\left(\omega^{+\bullet}-\omega^{+\bullet}\right) / \sqrt{2} i$. We now come to the remaining torsion components. From $T^{3}=0$ and $T^{1}=0$ one obtains respectively

$$
\omega^{+3}=A E^{-}, \quad \omega^{+1}=-\frac{E^{+}}{\ell \cosh \chi}+B E^{-},
$$

where $A$ and $B$ are some functions to be determined. Finally, $T^{+}=0$ yields

$$
\partial_{V} \mathcal{K}=2 \partial_{u} h_{0}, \quad A=-\frac{1}{2}\left(e^{4 \chi}-1\right) \frac{\sinh \chi}{\sqrt{\tanh \chi}} \partial_{\Psi} \mathcal{K}, \quad B=\frac{1}{\ell}\left(e^{4 \chi}-1\right) \sinh \chi \partial_{\chi} \mathcal{K} .
$$


The line element is given by

$$
\begin{aligned}
\mathrm{d} s^{2} & =2 E^{-} E^{+}+\left(E^{1}\right)^{2}+\left(E^{3}\right)^{2} \\
& =\operatorname{coth} \chi\left(\mathcal{K} \mathrm{d} u^{2}+2 h_{0} \mathrm{~d} u \mathrm{~d} V\right)+\frac{\ell^{2} \mathrm{~d} \chi^{2}}{4 \sinh ^{2} \chi}+\frac{\mathrm{d} \Psi^{2}}{\sinh \chi \cosh \chi} .
\end{aligned}
$$

As before, one can eliminate $h_{0}$ by introducing a new coordinate $v(u, V)$ with $\partial_{V} v=h_{0}$ and shifting $\mathcal{K} \rightarrow \mathcal{K}+2 \partial_{u} v$, whereupon the metric becomes

$$
\mathrm{d} s^{2}=\operatorname{coth} \chi\left(\mathcal{K} \mathrm{d} u^{2}+2 \mathrm{~d} u \mathrm{~d} v\right)+\frac{\ell^{2} \mathrm{~d} \chi^{2}}{4 \sinh ^{2} \chi}+\frac{\mathrm{d} \Psi^{2}}{\sinh \chi \cosh \chi} .
$$

Notice that, owing to (3.20), $\mathcal{K}$ is independent of $v$, therefore $\partial_{v}$ is a Killing vector. It coincides with the Killing vector $-\sqrt{2} D\left(\epsilon, \Gamma_{\mu} \epsilon\right)$ constructed from the Killing spinor. All that remains now is to impose Einstein's equations. One finds that they are all satisfied except for the $u$ component, which yields again a Siklos-type equation for $\mathcal{K}$,

$$
\partial_{\Psi}^{2} \mathcal{K}+4 \tanh \chi \partial_{\chi}^{2} \mathcal{K}-\frac{2}{\cosh ^{2} \chi} \partial_{\chi} \mathcal{K}=0 .
$$

In conclusion, the bosonic fields for a configuration admitting a null Killing spinor with $\mathrm{d} \chi \neq 0$ are given by (3.19) and (3.21), with $\mathcal{K}$ satisfying (3.22) $)^{6}$. As we will discuss in section 5.3, the $\mathcal{K}=0$ solution is of Petrov type $\mathrm{D}$ and represents a bubble of nothing in anti-De Sitter space-time. When $\mathcal{K} \neq 0$, the metric becomes of Petrov type II and the Weyl scalar signalling the presence of gravitational radiation acquires a non-vanishing value. Hence the general solution represents a gravitational wave on a bubble of nothing. To our knowledge these solutions have not featured in the literature before.

\subsection{Half-supersymmetric backgrounds}

In the previous subsections we have addressed the conditions for preserving one null Killing spinor of the form $\epsilon_{1}=1$ or $\epsilon_{1}=1+e^{x} e_{1}$. It is natural to enquire about the possibility of these backgrounds admitting an additional Killing spinor with the same $\mathbb{R}^{2}$ stability subgroup, i.e. of the form $\epsilon_{2}=c_{0} 1+c_{1} e_{1}$. Using the fact that $\epsilon_{1}$ is Killing, the second Killing spinor equation $D_{\mu} \epsilon_{2}=0$ can then be rewritten as

$$
\left(c_{0}-c_{1}\right) D_{\mu} 1+\partial_{\mu} c_{0} 1+\partial_{\mu} c_{1} e_{1}=0,
$$

\footnotetext{
${ }^{6}$ This solution escaped a majority of the present authors in [13]. The reason for this is that equ. (4.32) of [13] is not correct; it must be $R_{+-i j}=0$, which yields no information on the constant $\kappa$. Thus, in addition to the solutions with $\kappa=0$ found in [13] (the Lobachevski waves), there are also the $\kappa=1$ solutions, which are exactly the ones found here with $\mathrm{d} \chi \neq 0$.
} 
in the $\mathrm{U}(1) \ltimes \mathbb{R}^{2}$ case and

$$
\left(c_{0}-c_{1} e^{-\chi}\right) D_{\mu} 1+\partial_{\mu} c_{0} 1+\left(\partial_{\mu} c_{1}-c_{1} \partial_{\mu} \chi\right) e_{1}=0,
$$

in the $\mathbb{R}^{2}$ case. Furthermore, we can assume that $\left(c_{0}-c_{1}\right) \neq 0$ and $\left(c_{0}-c_{1} e^{-\chi}\right) \neq 0$ in the two cases, respectively, since otherwise the second Killing spinor would be linearly dependent on the first and there would not be any additional constraints. Hence the $e_{2}$ and $e_{12}$ components of $D_{\mu} 1$ have to vanish separately. In particular, this implies that $\omega^{-\bullet}=0$ (as can be seen from the third line of (3.1) in the singular limit $\chi \rightarrow-\infty$ ). However, this is clearly incompatible with (3.3). We conclude that, in the gauged theory, there are no backgrounds with four $\mathbb{R}^{2}$-invariant Killing spinors. In other words, there are no half-supersymmetric backgrounds with an $\mathbb{R}^{2}$-structure. This is unlike the ungauged case, where the half-supersymmetric gravitational waves provide such solutions.

Therefore, the only possibility to augment the supersymmetry of the null solutions above is to add a Killing spinor which breaks the $\mathbb{R}^{2}$ invariance, i.e. with a non-vanishing $e_{2}$ and/or $e_{12}$ component. From a linear combination of the first and second Killing spinor one can then always construct a time-like Killing spinor, and hence this brings us to the next section. For the convenience of the reader, we will already summarise how to restrict the 1/4-supersymmetric null solutions to allow for a time-like Killing spinor as well.

For the case with constant null Killing spinors, $\mathrm{d} \chi=0$, the restriction was already discussed in [13] and is given in (3.16). For the other case, with $\mathrm{d} \chi \neq 0$, it is straightforward to show that the solution (3.19), (3.21) admits a second Killing spinor iff $\partial_{\chi} \mathcal{G}=\partial_{\Psi} \mathcal{G}=0$, so that $\mathcal{G}$ depends only on $u$. By a simple diffeomorphism one can then set $\mathcal{G}=0$. The general solution to the Killing spinor equations reads in this case

$$
\epsilon=\lambda_{1}\left(1+e^{\chi} e_{1}\right)+\frac{\lambda_{2}}{\sqrt{e^{4 \chi}-1}}\left(e_{2}+e^{\chi} e_{1} \wedge e_{2}\right)
$$

where $\lambda_{1,2} \in \mathbb{C}$ are constants. The invariants constructed from $\epsilon$, as defined in appendix $\mathrm{B}$, are

$$
\begin{aligned}
& V=\sqrt{2} \operatorname{coth} \chi\left(\left|\lambda_{2}\right|^{2} \mathrm{~d} v-\left|\lambda_{1}\right|^{2} \mathrm{~d} u\right)-\frac{2 i}{\sinh 2 \chi}\left(\lambda_{2} \bar{\lambda}_{1}-\bar{\lambda}_{2} \lambda_{1}\right) \mathrm{d} \Psi \\
& B=-\sqrt{2}\left(\left|\lambda_{1}\right|^{2} \mathrm{~d} u+\left|\lambda_{2}\right|^{2} \mathrm{~d} v\right)+\frac{\ell e^{\chi}}{\sqrt{e^{4 \chi-1}} \sinh \chi}\left(\bar{\lambda}_{1} \lambda_{2}+\lambda_{1} \bar{\lambda}_{2}\right) \mathrm{d} \chi \\
& f=i\left(\lambda_{1} \bar{\lambda}_{2}-\bar{\lambda}_{1} \lambda_{2}\right) \sqrt{\tanh \chi}, \quad g=\left(\bar{\lambda}_{1} \lambda_{2}+\lambda_{1} \bar{\lambda}_{2}\right) \sqrt{\operatorname{coth} \chi}
\end{aligned}
$$

The norm of the Killing vector $V$ is given by

$$
V^{2}=-\frac{2}{\sinh 2 \chi}\left(\bar{\lambda}_{1} \lambda_{2}+\lambda_{1} \bar{\lambda}_{2}\right)^{2}-4\left|\lambda_{1} \lambda_{2}\right|^{2} \tanh \chi \text {. }
$$


Since $\chi>0$, this is negative unless $\lambda_{1}=0$ or $\lambda_{2}=0$, so indeed the solution (3.19),

(3.21) with $\mathcal{G}=0$ must belong also to the timelike class. It turns out that it is identical to the bubble of nothing of section 5.3 with imaginary $b$ and $L<0$. The coordinate transformation

$$
\begin{aligned}
& u=\sqrt{2} A^{2}(t-L y)-\frac{z}{2 \sqrt{2} A^{2}}, \quad v=-\sqrt{2} A^{2}(t-L y)-\frac{z}{2 \sqrt{2} A^{2}}, \\
& \Psi=-2 A^{2} t, \quad \chi=\operatorname{artanh} \frac{X^{2}}{A^{4}}
\end{aligned}
$$

with $A^{8}=-1 / 4 L$ brings the metric $(3.21)$ (with $\mathcal{G}=0$ ) to 5.60 ), and the field strength of (3.19) to (5.61). Note that, in the new coordinates, the above invariants become $V=\partial_{t}$ as a vector, and $B=\mathrm{d} z$, in agreement with section 4.2 .

\section{Timelike representative $1+b e_{2}$}

We will now turn to the timelike case and first recover the general 1/4-BPS solutions [13]. Afterwards we will study the conditions for $1 / 2$ supersymmetry. This will complete the classification since we already know that no 3/4-supersymmetric solutions can arise and $\mathrm{AdS}_{4}$ is the unique maximally supersymmetric possibility.

\subsection{Conditions from the Killing spinor equations}

Acting with the supercovariant derivative (2.5) on the representative $1+b e_{2}$ yields the linear system

$$
\begin{array}{r}
\partial_{+} b+\frac{b}{2} \omega_{+}^{\overline{\bullet \bullet}}-\frac{b}{2} \omega_{+}^{+-}-\frac{i}{\ell} b \mathcal{A}_{+}=0, \\
\frac{1}{2} \omega_{+}^{\overline{\bullet \bullet}}+\frac{1}{2} \omega_{+}^{+-}-\frac{i}{\ell} \mathcal{A}_{+}+\frac{b}{\ell \sqrt{2}}+\frac{i b}{\sqrt{2}} \mathcal{F}^{\overline{\bullet \bullet}}+\frac{i b}{\sqrt{2}} \mathcal{F}^{+-}=0, \\
\omega_{+}^{\bullet-}+i \sqrt{2} b \mathcal{F}^{\bullet-}=\omega_{+}^{\bullet+}=0, \\
\partial_{-} b+\frac{b}{2} \omega_{-}^{\mathbf{\bullet}-}-\frac{b}{2} \omega_{-}^{+-}-\frac{i}{\ell} b \mathcal{A}_{-}+\frac{1}{\ell \sqrt{2}}+\frac{i}{\sqrt{2}} \mathcal{F}^{\overline{\bullet \bullet}}-\frac{i}{\sqrt{2}} \mathcal{F}^{+-}=0, \\
\frac{1}{2} \omega_{-}^{\overline{\bullet \bullet}}+\frac{1}{2} \omega_{-}^{+-}-\frac{i}{\ell} \mathcal{A}_{-}=0, \\
b \omega_{-}^{\bullet+}+i \sqrt{2} \mathcal{F}^{\bullet+}=\omega_{-}^{\bullet-}=0,
\end{array}
$$




$$
\begin{aligned}
& \partial_{\bullet} b+\frac{b}{2} \omega_{\bullet}^{\bar{\bullet}}-\frac{b}{2} \omega_{\bullet}^{+-}-\frac{i}{\ell} b \mathcal{A}_{\bullet}-i \sqrt{2} \mathcal{F}^{\bar{\bullet}-}=0, \\
& \frac{1}{2} \omega_{\bullet}^{\bar{\bullet} \bullet}+\frac{1}{2} \omega_{\bullet}^{+-}-\frac{i}{\ell} \mathcal{A}_{\bullet}-i \sqrt{2} b \mathcal{F}^{\bar{\bullet}+}=0, \\
& \omega_{\bullet}^{\bullet-}+\frac{b}{\ell \sqrt{2}}-\frac{i b}{\sqrt{2}} \mathcal{F}^{\bar{\bullet} \bullet}-\frac{i b}{\sqrt{2}} \mathcal{F}^{+-}=0, \\
& b \omega_{\bullet}^{\bullet+}+\frac{1}{\ell \sqrt{2}}-\frac{i}{\sqrt{2}} \mathcal{F}^{\bar{\bullet} \bullet}+\frac{i}{\sqrt{2}} \mathcal{F}^{+-}=0, \\
& \partial_{\mathbf{\bullet}} b+\frac{b}{2} \omega_{\bar{\bullet}}^{\bar{\bullet} \bullet}-\frac{b}{2} \omega_{\bar{\bullet}}^{+-}-\frac{i}{\ell} b \mathcal{A}_{\mathbf{\bullet}}=0, \\
& \frac{1}{2} \omega_{\bar{\bullet}}^{\bar{\bullet}}+\frac{1}{2} \omega_{\bar{\bullet}}^{+-}-\frac{i}{\ell} \mathcal{A}_{\bar{\bullet}}=0, \\
& \omega_{\bullet}^{\bullet-}=b \omega_{\bullet}^{\bullet+}=0 \text {. }
\end{aligned}
$$

From eqns. (4.1) - (4.4) one obtains the gauge potential and the fluxes in terms of the spin connection and the function $b$,

$$
\begin{aligned}
\mathcal{A}_{+} & =\frac{i \ell}{2}\left(\frac{\partial_{+} \bar{b}}{\bar{b}}-\frac{\partial_{+} b}{b}-\omega_{+}^{\bar{\bullet}}\right), \quad \mathcal{A}_{-}=\frac{i \ell}{2} \omega_{-}^{\bullet \bullet}, \quad \mathcal{A}_{\bullet}=\frac{i \ell}{2}\left(\omega_{\bullet}^{\bullet \bar{\bullet}}+\omega_{\bullet}^{+-}\right), \\
\mathcal{F}^{+-} & =\frac{i}{\sqrt{2}}\left(b \omega_{\bullet}^{\bullet+}-b^{-1} \omega_{\bullet}^{\bullet-}\right), \quad \mathcal{F}^{\bullet+}=\frac{i}{\bar{b} \sqrt{2}} \omega_{\bullet}^{+-}, \\
\mathcal{F}^{\bullet-\bullet} & =\frac{i}{\sqrt{2}}\left(b \omega_{\bullet}^{\bullet+}+b^{-1} \omega_{\bullet}^{\bullet-}\right)+\frac{i}{\ell}, \quad \mathcal{F}^{\bullet-}=\frac{i}{b \sqrt{2}} \omega_{+}^{\bullet-} .
\end{aligned}
$$

Furthermore, the system (4.1) - (4.4) determines almost all components of the spin connection (with the exception of $\omega^{\bullet \boldsymbol{\bullet}}$ ) in terms of the function $b$ and its spacetime derivatives,

$$
\begin{aligned}
& \omega_{+}^{+-}=\frac{\partial_{+} b}{b}+\frac{\partial_{+} \bar{b}}{\bar{b}}, \quad \omega_{-}^{+-}=0, \quad \omega_{\bullet}^{+-}=\frac{\partial_{\bullet} \bar{b}}{\bar{b}}, \\
& \omega_{+}^{+\bullet}=\omega_{\bullet}^{+\bullet}=0, \quad \omega_{-}^{+\bullet}=-\frac{\partial_{\bullet} b}{b^{2} \bar{b}}, \quad \omega_{\bullet}^{+\bullet}=\frac{\partial_{-} b}{b}+\frac{\sqrt{2}}{b \ell}, \\
& \omega_{+}^{-\bullet}=-b \partial_{\bar{\bullet}} \bar{b}, \quad \omega_{-}^{-\bullet}=\omega_{-}^{-\bullet}=0, \quad \omega_{\bullet}^{-\bullet}=\frac{\partial_{+} \bar{b}}{\bar{b}}+\frac{b \sqrt{2}}{\ell} .
\end{aligned}
$$

In what follows, we assume $b \neq 0$. One easily shows that $b=0$ leads to $\ell^{-1}=0$, so this case appears only in ungauged supergravity.

\subsection{Geometry of spacetime}

In order to obtain the spacetime geometry, we consider the spinor bilinears

$$
V_{\mu}=D\left(\epsilon, \Gamma_{\mu} \epsilon\right), \quad B_{\mu}=D\left(\epsilon, \Gamma_{5} \Gamma_{\mu} \epsilon\right),
$$


whose nonvanishing components are

$$
V_{+}=\sqrt{2} \bar{b} b, \quad V_{-}=-\sqrt{2}, \quad B_{+}=\sqrt{2} \bar{b} b, \quad B_{-}=\sqrt{2} .
$$

As $V^{2}=-4 \bar{b} b=-B^{2}, V$ is timelike and $B$ is spacelike. Using eqns. (4.1) - (4.4), it is straightforward to show that $V$ is Killing and $B$ is closed, i. e. ,

$$
\begin{aligned}
\partial_{A} V_{B}+\partial_{B} V_{A}-\omega^{C}{ }_{B \mid A} V_{C}-\omega^{C}{ }_{A \mid B} V_{C} & =0, \\
\partial_{A} B_{B}-\partial_{B} B_{A}-\omega^{C}{ }_{B \mid A} B_{C}+\omega^{C}{ }_{A \mid B} B_{C} & =0 .
\end{aligned}
$$

There exists thus a function $z$ such that $B=d z$ locally. Let us choose coordinates $\left(t, z, x^{i}\right)$ such that $V=\partial_{t}$ and $i=1,2$. The metric will then be independent of $t$. Note also that the system (4.1) - (4.4) yields

$$
\partial_{t} b=\sqrt{2}\left(|b|^{2} \partial_{-}-\partial_{+}\right) b=0,
$$

so $b$ is time-independent as well. In terms of the vierbein $E_{\mu}^{A}$ the metric is given

$$
d s^{2}=2 E^{+} E^{-}+2 E^{\bullet} E^{\mathbf{\bullet}},
$$

where

$$
E_{\mu}^{+}=\frac{B_{\mu}+V_{\mu}}{2 \sqrt{2}|b|^{2}}, \quad E_{\mu}^{-}=\frac{B_{\mu}-V_{\mu}}{2 \sqrt{2}} .
$$

From $V^{2}=-4|b|^{2}$ and $V=\partial_{t}$ as a vector we get $V_{t}=-4|b|^{2}$, so that $V=-4|b|^{2}(d t+\sigma)$ as a one-form, with $\sigma_{t}=0$. Furthermore, $V^{\bullet}=0$ yields $E_{t}^{\bullet}=0$, and thus

$$
E^{\bullet}=E_{z}^{\bullet} d z+E_{i}^{\bullet} d x^{i}
$$

The component $E_{z}^{\bullet}$ can be eliminated by a diffeomorphism

$$
x^{i}=x^{i}\left(x^{\prime j}, z\right),
$$

with

$$
E_{i}^{I} \frac{\partial x^{i}}{\partial z}=-E_{z}^{I}, \quad I=\bullet, \bar{\bullet} .
$$

As the matrix $E_{i}^{I}$ is invertible ${ }^{7}$, one can always solve for $\partial x^{i} / \partial z$. Note that the metric is invariant under

$$
t \rightarrow t+\chi\left(x^{i}, z\right), \quad \sigma \rightarrow \sigma-d \chi
$$

\footnotetext{
${ }^{7}$ One has $\operatorname{det}\left(E_{i}^{I}\right)=-\operatorname{det}\left(E_{\mu}^{A}\right)$, and the latter is always nonzero.
} 
where $\chi\left(x^{i}, z\right)$ denotes an arbitrary function. This second gauge freedom can be used to eliminate $\sigma_{z}$. Hence, without loss of generality, we can take $\sigma=\sigma_{i} d x^{i}$, and the metric (4.7) becomes

$$
d s^{2}=-4|b|^{2}\left(d t+\sigma_{i} d x^{i}\right)^{2}+\frac{d z^{2}}{4|b|^{2}}+2 E_{i}^{\bullet} d x^{i} E_{j}^{\overline{\boldsymbol{\bullet}}} d x^{j}
$$

Next one has to impose vanishing torsion,

$$
\partial_{\mu} E_{\nu}^{A}-\partial_{\nu} E_{\mu}^{A}+\omega_{\mu B}^{A} E_{\nu}^{B}-\omega_{\nu B}^{A} E_{\mu}^{B}=0 .
$$

One finds that some of these equations are already identically satisfied, while the remaining ones yield (using the expressions (4.6) for the spin connection) the constraints

$$
\begin{aligned}
& \partial_{z} \sigma_{i}=-\frac{1}{4|b|^{2}}\left(E_{i}^{\overline{\boldsymbol{\bullet}}} E_{\bar{\bullet}}^{j}-E_{i}^{\bullet} E_{\bullet}^{j}\right) \partial_{j} \ln (b / \bar{b}), \\
& \partial_{i} \sigma_{j}-\partial_{j} \sigma_{i}=\left(E_{i}^{\bullet} E_{j}^{\overline{\mathbf{\bullet}}}-E_{j}^{\bullet} E_{i}^{\overline{\mathbf{\bullet}}}\right)\left(\partial_{z} \ln (b / \bar{b})+\frac{1}{b \ell}-\frac{1}{\bar{b} \ell}\right), \\
& \omega_{t}^{\bullet \bar{\bullet}}=-2|b|^{2} \partial_{z} \ln (b / \bar{b})+\frac{2 b}{\ell}-\frac{2 \bar{b}}{\ell},
\end{aligned}
$$

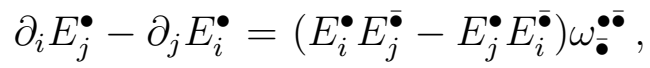

as well as

$$
\left[\partial_{z}+\omega_{z}^{\bullet \bar{\bullet}}+\frac{1}{2} \partial_{z} \ln (\bar{b} b)+\frac{1}{2 \ell}\left(\frac{1}{b}+\frac{1}{\bar{b}}\right)\right] E_{i}^{\bullet}=0 .
$$

In (4.9), $E_{I}^{i}$ denotes the inverse of $E_{j}^{J}$. In order to obtain the above equations, one has to make use of the inverse tetrad

$$
E_{+}=-\frac{1}{2 \sqrt{2}} \partial_{t}+\sqrt{2}|b|^{2} \partial_{z}, \quad E_{-}=\frac{1}{2 \sqrt{2}|b|^{2}} \partial_{t}+\sqrt{2} \partial_{z}, \quad E_{\bullet}=E_{\bullet}^{i}\left(\partial_{i}-\sigma_{i} \partial_{t}\right) .
$$

(4.13) can be solved to give

$$
E_{i}^{\bullet}=\frac{1}{|b|} \hat{E}_{i}^{\bullet} \exp \left[-\int d z \omega_{z}^{\bullet \bullet}-\frac{1}{2 \ell} \int d z\left(\frac{1}{b}+\frac{1}{\bar{b}}\right)\right]
$$

where $\hat{E}_{i}^{\bullet}$ is an integration constant that depends only on the coordinates $x^{j}$. At this point it is convenient to use the residual $\mathrm{U}(1)$ gauge freedom of a combined local Lorentz and gauge transformation to eliminate $\omega_{z}^{\bullet \cdot}$. This is accomplished by the transformation (2.3), with

$$
\psi=\frac{i}{2} \int d z \omega_{z}^{\bullet \bullet}
$$


Note that $\psi$ is real, as it must be. Defining

$$
\Phi:=-\frac{1}{2 \ell} \int d z\left(\frac{1}{b}+\frac{1}{\bar{b}}\right)
$$

we have thus

$$
E_{i}^{\bullet}=\frac{1}{|b|} \hat{E}_{i}^{\bullet} \exp \Phi
$$

Using (4.16) in (4.12), one gets for the only remaining unknown component $\omega_{\bullet}^{\bullet \bullet}$ of the spin connection

$$
\omega_{\bullet}^{\bullet \bar{\bullet}}=\left[\hat{\omega}_{\bullet}^{\bullet \bar{\bullet}}-\hat{E}_{\bullet}^{i} \partial_{i}\right]|b| \exp (-\Phi)
$$

where $\hat{\omega}_{\bullet}^{\bullet \bullet}$ denotes the spin connection following from the zweibein $\hat{E}_{i}^{I}$.

In what follows, we shall choose the conformal gauge for the two-metric $h_{i j}=$ $\hat{E}_{I i} \hat{E}_{j}^{I}$, i. e. ,

$$
h_{i j}=e^{2 \xi}\left[\left(d x^{1}\right)^{2}+\left(d x^{2}\right)^{2}\right] .
$$

with $\xi$ depending only on the coordinates $x^{i}$. Furthermore, we choose an orientation such that

$$
\hat{E}_{i}^{\bullet} \hat{E}_{j}^{\bar{\bullet}}-\hat{E}_{j}^{\bullet} \hat{E}_{i}^{\bar{\bullet}}=-i e^{2 \xi} \epsilon_{i j}
$$

where $\epsilon_{12}=1$. To be concrete, we shall take

$$
\left(\hat{E}_{i}^{I}\right)=\frac{1}{\sqrt{2}} e^{\xi}\left(\begin{array}{rr}
1 & i \\
1 & -i
\end{array}\right) .
$$

The eqns. (4.9) and (4.10) then simplify to

$$
\begin{aligned}
\partial_{z} \sigma_{i} & =-\frac{i}{4|b|^{2}} \epsilon_{i j} \partial_{j} \ln (b / \bar{b}) \\
\partial_{i} \sigma_{j}-\partial_{j} \sigma_{i} & =-\frac{i}{|b|^{2}} e^{2(\Phi+\xi)} \epsilon_{i j}\left(\partial_{z} \ln (b / \bar{b})+\frac{1}{b \ell}-\frac{1}{\bar{b} \ell}\right) .
\end{aligned}
$$

Moreover, one has

$$
\omega_{\bullet}^{\bullet \bar{\bullet}}=-\partial_{\bullet} \ln \left(|b| e^{-\Phi-\xi}\right) .
$$

In [13] it has been shown that in the case where the Killing vector constructed from the Killing spinor is timelike, the Einstein equations follow from the Killing spinor equations, so all that remains to do at this point is to impose the Bianchi identity and the Maxwell equations. Using the spin connection (4.6) and (4.11) in (4.5), the gauge 
potential and the field strength become

$$
\begin{aligned}
\mathcal{A}= & i(d t+\sigma)(b-\bar{b})+\frac{\ell}{2} \epsilon_{i j} \partial_{j}(\Phi+\xi) d x^{i}-\frac{i \ell}{4} d \ln (b / \bar{b}), \\
\mathcal{F}= & i(d t+\sigma) \wedge d(\bar{b}-b)+\frac{1}{4|b|^{2}} d z \wedge d x^{i} \epsilon_{i j} \partial_{j}(b+\bar{b}) \\
& +\frac{1}{2|b|^{2}}\left[\partial_{z}(b+\bar{b})+\frac{1}{\ell}\right] e^{2(\Phi+\xi)} \epsilon_{i j} d x^{i} \wedge d x^{j} .
\end{aligned}
$$

The Bianchi identity $\mathcal{F}=d \mathcal{A}$ yields

$$
\Delta(\Phi+\xi)=\frac{2}{\ell} e^{2(\Phi+\xi)}\left[\partial_{z} \frac{1}{b}+\partial_{z} \frac{1}{\bar{b}}-\frac{1}{b^{2} \ell}-\frac{1}{\bar{b}^{2} \ell}+\frac{1}{\bar{b} b \ell}\right]
$$

with $\Delta=\partial_{i} \partial_{i}$ denoting the flat space Laplacian in two dimensions. As for the Maxwell equations,

$$
\partial_{\mu}\left(\sqrt{-g} \mathcal{F}^{\mu \nu}\right)=0
$$

the only nontrivial information comes from the $t$-component, which gives

$$
4 e^{2(\Phi+\xi)}\left[b^{2} \partial_{z}^{2} \frac{1}{b}-\bar{b}^{2} \partial_{z}^{2} \frac{1}{\bar{b}}-\frac{3 b}{\ell} \partial_{z} \frac{1}{b}+\frac{3 \bar{b}}{\ell} \partial_{z} \frac{1}{\bar{b}}+\frac{1}{b \ell^{2}}-\frac{1}{\bar{b} \ell^{2}}\right]+b^{2} \Delta \frac{1}{b}-\bar{b}^{2} \Delta \frac{1}{\bar{b}}=0,
$$

where we used eqns. (4.18) and (4.19).

Let us now show that the equations (4.22) and (4.23) are actually the same as the ones in [16]. If we set

$$
F=-\frac{1}{\ell \bar{b}}, \quad e^{\phi}=2 e^{\Phi+\xi}
$$

(4.22) yields exactly equation (2.3) of [16]. On the other hand, deriving (4.22) with respect to $z$ and using (4.15), one obtains

$$
\Delta A+e^{2 \phi}\left[3 A \partial_{z} A-3 B \partial_{z} B+A^{3}-3 A B^{2}+\partial_{z}^{2} A\right]=0,
$$

where $A$ and $B$ denote the real and imaginary part of $F$ respectively. This can be used in (4.23) to get

$$
\Delta B+e^{2 \phi}\left[\partial_{z}^{2} B+3 B \partial_{z} A+3 A \partial_{z} B-B^{3}+3 A^{2} B\right]=0
$$

which, together with (4.25), yields

$$
\Delta F+e^{2 \phi}\left[F^{3}+3 F \partial_{z} F+\partial_{z}^{2} F\right]=0,
$$

i. e. , equation (2.2) of [16]. For a complete identification of the present results with the ones in [16], one also has to set $\sigma=\omega$. 
In conclusion, the metric of the general 1/4-supersymmetric solution is given by

$$
d s^{2}=-4|b|^{2}(d t+\sigma)^{2}+\frac{1}{4|b|^{2}}\left(d z^{2}+4 e^{2(\Phi+\xi)} d w d \bar{w}\right)
$$

where $b$ and $\phi$ are determined by the system (4.22), (4.23) and $w=x^{1}+i x^{2} \equiv x+i y$. The one-form $\sigma$ follows then from (4.18) and (4.19), and the gauge field strength is given by (4.21). Note that (4.23) represents also the integrability condition for (4.18), (4.19). As noted in [16], this system of equations is invariant under $\operatorname{PSL}(2, \mathbb{R})$ transformations $^{8}$. If we define a new coordinate $z^{\prime}$ through the Möbius transformation

$$
z^{\prime}=\frac{\alpha z+\beta}{\gamma z+\delta}
$$

with $\alpha, \beta, \gamma$ and $\delta$ arbitrary real constants satisfying $\alpha \delta-\beta \gamma=1$, then the functions $\tilde{b}\left(z^{\prime}, x^{i}\right)$ and $\tilde{\Phi}\left(z^{\prime}, x^{i}\right)$ defined by

$$
\frac{1}{\tilde{b}}=\frac{1}{\left(\gamma z^{\prime}-\alpha\right)^{2} b}-\frac{2 l \gamma}{\gamma z^{\prime}-\alpha}, \quad e^{\tilde{\Phi}}=\left(\gamma z^{\prime}-\alpha\right)^{2} e^{\Phi},
$$

solve the system in the new coordinate system $\left(z^{\prime}, x^{i}\right)$, with the function $\xi\left(x^{i}\right)$ left invariant and $z$ seen as a function of $z^{\prime}$. This symmetry allows to generate new BPS solutions from the known ones. Note however that it is only a symmetry of the equations for $1 / 4$ supersymmetry, and if we apply it to solutions with additional Killing spinors, it will in general not preserve them, as we shall show explicitely in some examples.

\subsection{Half-supersymmetric backgrounds}

We now would like to investigate the possibility of adding a second Killing spinor. Since the first Killing spinor $\epsilon_{1}$ has stability subgroup 1, one cannot use Lorentz transformations to bring the second spinor to a preferred form. Therefore we use the most general form

$$
\epsilon_{2}=c_{0} 1+c_{1} e_{1}+c_{2} e_{2}+c_{12} e_{1} \wedge e_{2} .
$$

The corresponding linear system simplifies significantly after inserting the results from $\epsilon_{1}$. These determine all the fluxes and the spin connection in terms of the functions $b$, $\xi$ and their derivatives. First it is convenient to introduce the new basis ${ }^{9}$

$$
\alpha=\left(\begin{array}{c}
\alpha_{0} \\
\alpha_{1} \\
\alpha_{2} \\
\alpha_{12}
\end{array}\right)=\left(\begin{array}{c}
c_{0} \\
b^{-1} c_{2}-c_{0} \\
\bar{b} c_{1} \\
c_{12}
\end{array}\right),
$$

\footnotetext{
${ }^{8}$ It might be of interest to investigate the possible relation between this 'hidden symmetry' and the Ehlers group for solutions of four-dimensional vacuum gravity with a Killing vector.

${ }^{9}$ Note that $\epsilon_{1}=(1,0,0,0)$ in this basis.
} 
in which the Killing spinor equations for $\epsilon_{2}$ read

$$
\left(\partial_{A}+M_{A}\right) \alpha=0
$$

with the connection $M_{A}$ given by

$$
\begin{aligned}
& M_{+}=\left(\begin{array}{cccc}
0 & -\partial_{+} \ln \bar{b} & 0 & 0 \\
0 & \partial_{+} \ln \bar{b} & -\partial_{\bullet} \ln b & \partial_{\bullet} \ln b \\
0 & 0 & \frac{\bar{b}-b}{\sqrt{2} \ell}+\frac{1}{2} \partial_{+} \ln \frac{b}{b} & -\frac{\sqrt{2}}{\ell} \bar{b}-\partial_{+} \ln b \\
0 & -|b|^{2} \partial_{\bullet} \ln \bar{b} & 0 & \frac{\bar{b}-b}{\sqrt{2} \ell}-\frac{1}{2} \partial_{+} \ln (\bar{b} b)
\end{array}\right), \\
& M_{-}=\left(\begin{array}{cccc}
0 & 0 & |b|^{-2} \partial_{\bullet} \ln \bar{b} & -|b|^{-2} \partial_{\bullet} \ln \bar{b} \\
0 & \partial_{-} \ln b & -|b|^{-2} \partial_{\bullet} \ln \bar{b} & |b|^{-2} \partial_{\bullet} \ln \bar{b} \\
0 & \partial_{\bullet} \ln b & \frac{b-\bar{b}}{\sqrt{2} \ell|b|^{2}}-\frac{1}{2} \partial_{-} \ln (\bar{b} b) & 0 \\
0 & 0 & -\frac{\sqrt{2}}{\ell b}-\partial_{-} \ln \bar{b} & \frac{b-\bar{b}}{\sqrt{2} \ell|b|^{2}}+\frac{1}{2} \partial_{-} \ln \frac{\bar{b}}{b}
\end{array}\right), \\
& M_{\bullet}=\left(\begin{array}{cccc}
0 & -\partial_{\bullet} \ln \bar{b} & 0 & 0 \\
0 & \partial_{\bullet} \ln (\bar{b} b) & 0 & 0 \\
0 & -\frac{\sqrt{2}}{\ell} \bar{b}-\partial_{+} \ln b & -\partial_{\bullet} \ln \left(|b| e^{-\Phi-\xi}\right) & 0 \\
0 & \frac{\sqrt{2}}{\ell} b+\partial_{+} \ln \bar{b} & 0 & -\partial_{\bullet} \ln \left(|b| e^{-\Phi-\xi}\right)
\end{array}\right) \text {, } \\
& M_{\bullet}=\left(\begin{array}{cccc}
0 & 0 & -\partial_{-} \ln \bar{b} & \partial_{-} \ln \bar{b}+\frac{\sqrt{2}}{\ell b} \\
0 & 0 & \partial_{-} \ln (\bar{b} b)+\frac{\sqrt{2}}{\ell b} & -\partial_{-} \ln (\bar{b} b)-\frac{\sqrt{2}}{\ell \bar{b}} \\
0 & 0 & \partial_{\boldsymbol{\bullet}} \ln \left(\sqrt{\frac{b}{b}} e^{-\Phi-\xi}\right) & -\partial_{\mathbf{\bullet}} \ln b \\
0 & 0 & -\partial_{\mathbf{\bullet}} \ln \bar{b} & \partial_{\mathbf{\bullet}} \ln \left(\sqrt{\frac{\bar{b}}{b}} e^{-\Phi-\xi}\right)
\end{array}\right) .
\end{aligned}
$$

Let us first of all consider the simpler possibility of a second Killing spinor of the form $\epsilon_{2}=c_{0} 1+c_{2} e_{2}$. As discussed in section 2.1, both $\epsilon_{1}$ and $\epsilon_{2}$ are invariant under the same $\mathrm{U}(1)$ symmetry, and hence this case constitutes the $G=\mathrm{U}(1)$ case with four supersymmetries. As can easily be seen from the above Killing spinor equations with $\alpha_{1} \neq 0$ and $\alpha_{2}=\alpha_{12}=0$, this restricts the derivatives of the coefficient $b$ to be

$$
\partial_{-} b=-\frac{\sqrt{2}}{\ell}, \quad \partial_{+} b=-\frac{\sqrt{2} b \bar{b}}{\ell}, \quad \partial_{\bullet} b=\partial_{\boldsymbol{\bullet}} b=0 .
$$

Hence this corresponds to $\partial_{z} b=-1 / \ell$. As will be discussed in section 5.1 , this restriction uniquely leads to the half-supersymmetric anti-Nariai space-time. Hence $A d S_{2} \times H^{2}$ is the only possibility for backgrounds with four $\mathrm{U}(1)$-invariant Killing spinors.

In the more general case with $\alpha_{2}$ and $\alpha_{12}$ non-vanishing, i.e. with trivial stability subgroup, the Killing spinor equations do not so readily provide information about $b$ 
and one has to resort to their integrability conditions. The first integrability conditions for the linear system (4.31) are

$$
N_{\mu \nu} \alpha \equiv\left(\partial_{\mu} M_{\nu}-\partial_{\nu} M_{\mu}+\left[M_{\mu}, M_{\nu}\right]\right) \alpha=0
$$

where the matrices $M_{\mu}=E_{\mu}^{A} M_{A}$ are given by

$$
\begin{gathered}
M_{t}=\sqrt{2}\left(|b|^{2} M_{-}-M_{+}\right), \quad M_{z}=\frac{1}{2 \sqrt{2}|b|^{2}}\left(M_{+}+|b|^{2} M_{-}\right), \\
M_{w}=\sigma_{w} M_{t}+\frac{1}{\sqrt{2}|b|} e^{\Phi+\xi} M_{\bullet}, \quad M_{\bar{w}}=\sigma_{\bar{w}} M_{t}+\frac{1}{\sqrt{2}|b|} e^{\Phi+\xi} M_{\mathbf{\bullet}},
\end{gathered}
$$

and we introduced the complex coordinates $w=x+i y, \bar{w}=x-i y$. For halfsupersymmetric solutions, the six matrices $N_{\mu \nu}$ must have rank two. (As at least one Killing spinor exists, namely $\epsilon_{1}=(1,0,0,0)$, we already know that the $N_{\mu \nu}$ can have at most rank three. Rank one is not possible, because 3/4 BPS solutions cannot exist [29]. Rank zero corresponds to the maximally supersymmetric case, which implies that the spacetime geometry is $\mathrm{AdS}_{4}$ [13].) Let us define

$$
\tilde{N}_{\mu \nu} \equiv S N_{\mu \nu} T
$$

with

$$
S=\left(\begin{array}{llll}
1 & 0 & 0 & 0 \\
1 & 1 & 0 & 0 \\
0 & 0 & 1 & 0 \\
0 & 0 & 0 & 1
\end{array}\right), \quad T=\left(\begin{array}{llll}
1 & 0 & 0 & 0 \\
0 & 1 & 0 & 0 \\
0 & 0 & 1 & 0 \\
0 & 0 & 1 & 1
\end{array}\right)
$$

The similarity transformation $S$ corresponds to adding the first line to the second one and $T$ adds the last column to the third one. This does not alter the rank of $N_{\mu \nu}$. One finds

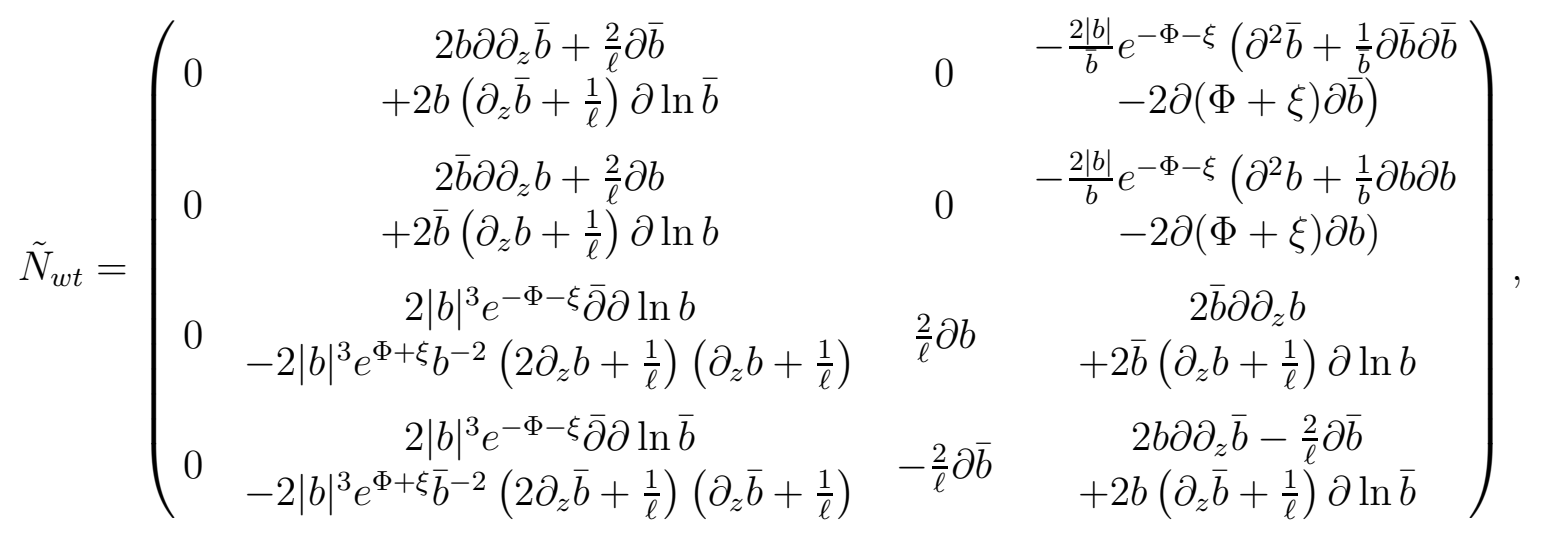




$$
\begin{aligned}
& \tilde{N}_{\bar{w} t}= \\
& \left(\begin{array}{ccc}
0 & 2 b \bar{\partial} \partial_{z} \bar{b} & -2|b| e^{-\Phi-\xi} \bar{\partial} \partial \ln \bar{b} \\
+2 b\left(\partial_{z} \bar{b}+\frac{1}{\ell}\right) \bar{\partial} \ln \bar{b} & -\frac{2}{\ell|b|} e^{\Phi+\xi}\left(2 \partial_{z} \bar{b}+\frac{1}{\ell}\right) & +\frac{2 b}{|b| \bar{b}} e^{\Phi+\xi}\left(2 \partial_{z} \bar{b}+\frac{1}{\ell}\right)\left(\partial_{z} \bar{b}+\frac{b-\bar{b}}{\ell b}\right) \\
2 \bar{b} \bar{\partial} \partial_{z} b & & -2|b| e^{-\Phi-\xi} \bar{\partial} \partial \ln b \\
0 & \frac{2}{\ell|b|} e^{\Phi+\xi}\left(2 \partial_{z} b+\frac{1}{\ell}\right) & +\frac{2 \bar{b}}{|b| b} e^{\Phi+\xi}\left(2 \partial_{z} b+\frac{1}{\ell}\right)\left(\partial_{z} b+\frac{1}{\ell}\right) \\
+2 \bar{b}\left(\partial_{z} b+\frac{1}{\ell}\right) \bar{\partial} \ln b & & 2 \bar{b} \bar{\partial} \partial_{z} b+\frac{2}{\ell} \bar{\partial} b \\
0 & 2|b| \bar{b} e^{-\Phi-\xi}\left(\bar{\partial}^{2} b+\frac{1}{b} \bar{\partial} b \bar{\partial} b\right. & +2 \bar{b}\left(\partial_{z} b+\frac{1}{\ell}\right) \bar{\partial} \ln b \\
-2 \bar{\partial}(\Phi+\xi) \bar{\partial} b) & -\frac{6}{\ell} \bar{\partial} \bar{b} & 2 b \bar{\partial} \partial_{z} \bar{b}-\frac{4}{\ell} \bar{\partial} \bar{b} \\
0 & 2|b| b e^{-\Phi-\xi}\left(\bar{\partial}^{2} \bar{b}+\frac{1}{b} \bar{\partial} \bar{b} \bar{\partial} \bar{b}\right. & +2 b\left(\partial_{z} \bar{b}+\frac{1}{\ell}\right) \bar{\partial} \ln \bar{b}
\end{array}\right),
\end{aligned}
$$

where $\partial=\partial_{w}, \bar{\partial}=\partial_{\bar{w}}$. The other four integrability conditions give no additional information, because the lines of the corresponding matrices are proportional to the lines of $\tilde{N}_{w t}$ and $\tilde{N}_{\bar{w} t}{ }^{10}$.

As the upper right $3 \times 3$ determinant of $\tilde{N}_{w t}$ must vanish, we obtain $\partial b=0$ or

$$
\partial_{z}\left(e^{-2(\Phi+\xi)} \bar{b} \partial \bar{b}\right) \partial\left(e^{-2(\Phi+\xi)} b \partial b\right)-\partial_{z}\left(e^{-2(\Phi+\xi)} b \partial b\right) \partial\left(e^{-2(\Phi+\xi)} \bar{b} \partial \bar{b}\right)=0 \text {. }
$$

Let us assume that the expression in (4.34) does not vanish. One has then $\partial b=0$ as well as $\partial \bar{b}=0^{11}$. But then also (4.34) holds, which leads to a contradiction. Thus (4.34) must be satisfied in any case.

Note that the vanishing of the first column of $\tilde{N}_{\mu \nu}$ implies that also the first column of $T^{-1} N_{\mu \nu} T$ is zero, and thus $T^{-1} N_{\mu \nu} T \in \mathrm{a}(3, \mathbb{C})$, hence the generalized holonomy in the case of one preserved complex supercharge is contained in the affine group $\mathrm{A}(3, \mathbb{C})$. This supports the classification scheme of [4]. Of course, depending on the particular solution, the generalized holonomy may also be a subgroup of $\mathrm{A}(3, \mathbb{C})$.

\subsection{Time-dependence of second Killing spinor}

In this section we will utilize the above Killing spinor equations to derive the timedependence of the second Killing spinor. In addition, we will show that the Killing spinor equations can be completely solved when the second Killing spinor is timeindependent.

Let us first simplify the Killing spinor equations (4.31). In the following we set $b=r e^{i \varphi}$ and define $\psi=\Phi+\xi, \psi_{1}=r^{2} \alpha_{1}, \psi_{2}=r e^{-\psi} \alpha_{2}, \psi_{12}=r e^{-\psi} \alpha_{12}$ and $\psi_{ \pm}=$

\footnotetext{
${ }^{10}$ In order to show this, one has to make use of eqns. (4.22) and $(4.26)$.

${ }^{11}$ This follows from the vanishing of the $3 \times 3$ determinant that is obtained from $\tilde{N}_{w t}$ by deleting the first column and the third line.
} 
$\psi_{2} \pm \psi_{12}$. First of all, use the integrability conditions (4.33), that can be rewritten as $\tilde{N}_{\mu \nu} T^{-1} \alpha=0$. Defining $P=e^{-2 \psi} b \partial b$, the second component for $\mu=w, \nu=t$ gives

$$
\psi_{1} P^{\prime}+\psi_{-} \partial P=0,
$$

with ${ }^{\prime}=\partial_{z}$. Let us assume $P^{\prime} \neq 0$ (the case $P^{\prime}=0$ is considered in appendix $\overline{9}$ and will lead to the same conclusions). If we define $g(t, z, w, \bar{w})=-\psi_{-} / P^{\prime}$, we get

$$
\psi_{-}=-g P^{\prime}, \quad \psi_{1}=g \partial P .
$$

The third component of the $(w, t)$ integrability condition is of the form

$$
\psi_{1} f_{1}+\psi_{2} \partial b+\psi_{-} f_{-}=0,
$$

for some functions $f_{1}, f_{-}$that depend on $z, w, \bar{w}$ but not on $t$. Using the above form of $\psi_{1}$ and $\psi_{-}$, this becomes

$$
f_{1} g \partial P+\psi_{2} \partial b-f_{-} g P^{\prime}=0 .
$$

Now, if $g=0$, the latter equation implies $\psi_{2} \partial b=0$, and hence (since $\partial b \neq 0$ due to $\left.P^{\prime} \neq 0\right) \psi_{2}=0$. Furthermore, $\psi_{1}=\psi_{-}=0$ in this case, so there exists no other Killing spinor. Thus, $g \neq 0$ and we can write $g=\exp G$. Dividing (4.36) by $g$ and deriving with respect to $t$ yields $\partial_{t}\left(\psi_{2} / g\right)=0$ and hence

$$
\psi_{2}=e^{G} \psi_{2}^{0}(z, w, \bar{w}) .
$$

It is then plain that $\partial_{t} \psi_{i}=\psi_{i} \partial_{t} G, i=1,2,12$. The Killing spinor equations are of the form $\partial_{\mu} \psi_{i}=\mathcal{M}_{\mu i j} \psi_{j}$, for some time-independent matrices $\mathcal{M}_{\mu}$. Taking the derivative of this with respect to $t$, one gets $\partial_{\mu} \partial_{t} G=0$, whence

$$
G=G_{0} t+\tilde{G}(z, w, \bar{w}),
$$

with $G_{0} \in \mathbb{C}$ constant. We have thus $\partial_{t} \psi_{i}=G_{0} \psi_{i}$ and hence also $\partial_{t} \alpha_{i}=G_{0} \alpha_{i}$. Furthermore, the time-dependence of $\alpha_{0}$ can be easily deduced from the Killing spinor equations: if $G_{0}$ does not vanish it is of the same exponential form as the other components of the second Killing spinor, i.e. $\partial_{t} \alpha_{0}=G_{0} \alpha_{0}$, while if $G_{0}$ vanishes there can be a linear part in $t$, i.e. $\partial_{t} \alpha_{0}=c$ for some constant $c$. Hence, in terms of the basis elements, the time-dependence of the second Killing spinor takes the form ${ }^{12}$

$$
\begin{array}{ll}
G_{0}=0: & \epsilon_{2}=c_{0} 1+c_{1} e_{1}+c_{2} e_{2}+c_{12} e_{1} \wedge e_{2}+c t\left(1+b e_{2}\right), \\
G_{0} \neq 0: & \epsilon_{2}=e^{G_{0} t}\left(c_{0} 1+c_{1} e_{1}+c_{2} e_{2}+c_{12} e_{1} \wedge e_{2}\right),
\end{array}
$$

\footnotetext{
${ }^{12}$ We will loosely refer to Killing spinors with $G_{0}=0$ as time-independent, despite the possible linear time-dependence, to distinguish from the $G_{0} \neq 0$ exponential time-dependence.
} 
where $c_{0}, c_{1}, c_{2}, c_{12}$ are time-independent functions of the spatial coordinates, and $c$ is a constant. This was derived assuming $P^{\prime}$ does not vanish, but as we show in appendix $\mathrm{C}$ is in fact a completely general result. Hence, adding a second Killing spinor to $\epsilon_{1}=1+b e_{2}$, the Killing spinor equations imply that $\epsilon_{2}$ always has the above timedependence.

Plugging this time-dependence into the subsystem of the Killing spinor equations not containing $\alpha_{0}$ one obtains in terms of $\psi_{i}$

$$
\begin{aligned}
& \psi_{1}^{\prime}-\left(\frac{G_{0}}{4 r^{2}}+\frac{b^{\prime}}{b}\right) \psi_{1}-\frac{\partial b}{b} \psi_{-}=0, \\
& \psi_{2}^{\prime}-\left(\frac{G_{0}}{4 r^{2}}+\frac{\bar{b}^{\prime}}{\bar{b}}+\frac{1}{\ell \bar{b}}\right) \psi_{2}-\left(\frac{b^{\prime}}{b}+\frac{1}{\ell b}\right) \psi_{12}=0 \text {, } \\
& \psi_{12}^{\prime}-e^{-2 \psi} \frac{\bar{\partial} \bar{b}}{\bar{b}} \psi_{1}-\left(\frac{G_{0}}{4 r^{2}}+\frac{b^{\prime}}{b}+\frac{\bar{b}^{\prime}}{\bar{b}}+\frac{1}{\ell \bar{b}}\right) \psi_{12}=0, \\
& \psi_{1}^{\prime}-\left(-\frac{G_{0}}{4 r^{2}}+\frac{\bar{b}^{\prime}}{\bar{b}}\right) \psi_{1}-\frac{\partial \bar{b}}{\bar{b}} \psi_{-}=0, \\
& \psi_{2}^{\prime}+e^{-2 \psi} \frac{\bar{\partial} b}{b} \psi_{1}-\left(-\frac{G_{0}}{4 r^{2}}+\frac{b^{\prime}}{b}+\frac{\bar{b}^{\prime}}{\bar{b}}+\frac{1}{\ell b}\right) \psi_{2}=0, \\
& \psi_{12}^{\prime}-\left(\frac{\bar{b}^{\prime}}{\bar{b}}+\frac{1}{\ell \bar{b}}\right) \psi_{2}-\left(-\frac{G_{0}}{4 r^{2}}+\frac{b^{\prime}}{b}+\frac{1}{\ell b}\right) \psi_{12}=0, \\
& \partial \psi_{1}-\sigma_{w} G_{0} \psi_{1}=0 \\
& \partial \psi_{2}-\left(\frac{b^{\prime}}{b}+\frac{1}{\ell b}\right) \psi_{1}-\left(\sigma_{w} G_{0}+\frac{\partial b}{b}+\frac{\partial \bar{b}}{\bar{b}}-2 \partial \psi\right) \psi_{2}=0 \\
& \partial \psi_{12}+\left(\frac{\bar{b}^{\prime}}{\bar{b}}+\frac{1}{\ell \bar{b}}\right) \psi_{1}-\left(\sigma_{w} G_{0}+\frac{\partial b}{b}+\frac{\partial \bar{b}}{\bar{b}}-2 \partial \psi\right) \psi_{12}=0 \\
& \bar{\partial} \psi_{1}-\left(\sigma_{\bar{w}} G_{0}+\frac{\bar{\partial} b}{b}+\frac{\bar{\partial} \bar{b}}{\bar{b}}\right) \psi_{1}+e^{2 \psi}\left[\left(\frac{b^{\prime}}{b}+\frac{\bar{b}^{\prime}}{\bar{b}}\right) \psi_{-}+\frac{1}{\ell}\left(\frac{\psi_{2}}{b}-\frac{\psi_{12}}{\bar{b}}\right)\right]=0 \\
& \bar{\partial} \psi_{2}-\left(\sigma_{\bar{w}} G_{0}+\frac{\bar{\partial} \bar{b}}{\bar{b}}\right) \psi_{2}-\frac{\bar{\partial} b}{b} \psi_{12}=0 \\
& \bar{\partial} \psi_{12}-\frac{\bar{\partial} \bar{b}}{\bar{b}} \psi_{2}-\left(\sigma_{\bar{w}} G_{0}+\frac{\bar{\partial} b}{b}\right) \psi_{12}=0
\end{aligned}
$$

For $G_{0}=0$, these equations simplify significantly, and allow for a complete solution. As is shown in appendix $\mathrm{D}$, under the additional assumption $\psi_{-} \neq 0, \psi_{1} \neq 0$, the metric 
and the field strength for half-supersymmetric solutions with $G_{0}=0$ are given in terms of a single real function $H$ depending only on the combination $Z-w-\bar{w}$ and satisfying the second order differential equation

$$
2\left(1+e^{-2 H}\right) \ddot{H}+\dot{H}^{2}\left[1-\frac{3 \alpha^{2}}{e^{2 H}+1-\alpha^{2}}\right]=\frac{\gamma^{2}}{\ell^{2}},
$$

where $\alpha \in \mathbb{R}$ denotes an arbitrary constant and $\gamma=0,1$. The new coordinate $Z$ is defined by $Z=z$ for $\gamma=0$ and $Z=\ell \ln \left(1+\frac{z}{\ell}\right)$ for $\gamma=1$. Furthermore, in the remainder of this section and in appendix D, a dot denotes a derivative with respect to $Z-w-\bar{w}$. Given a solution of (4.50), one defines the functions $\chi, \rho$ by

$$
\chi=\frac{i \alpha}{\sqrt{e^{2 H}+1-\alpha^{2}}}, \quad \frac{1}{\ell^{2} \rho^{2}}=\left(\frac{\gamma}{\ell}+\dot{H}\right)^{2}-\dot{H}^{2} \chi^{2}
$$

Note that $\chi$ is imaginary and $\rho$ is real. $b$ and $\psi$ are then given by

$$
b=e^{\gamma Z / \ell} \rho e^{i \varphi}, \quad e^{2 \psi}=e^{2(H+\gamma Z / \ell)},
$$

where

$$
\tan \varphi=\frac{i \dot{H} \chi}{\frac{\gamma}{\ell}+\dot{H}}
$$

so that the metric reads

$$
d s^{2}=-4 \rho^{2} e^{2 \gamma Z / \ell}(d t+\sigma)^{2}+\frac{1}{\rho^{2}}\left(\frac{d Z^{2}}{4}+e^{2 H} d w d \bar{w}\right),
$$

where the shift vector satisfies

$$
\partial_{Z} \sigma_{w}=\frac{1}{4} e^{-\gamma Z / \ell}\left(\frac{\chi}{\rho^{2}}\right), \quad \partial \sigma_{\bar{w}}-\bar{\partial} \sigma_{w}=-\frac{1}{2} e^{-\gamma Z / \ell}\left(e^{2 H} \frac{\chi}{\rho^{2}}\right) .
$$

Finally, the gauge field strength is given by (4.21).

Equation (4.50) is actually the Euler-Lagrange equation for the following standard action for the scalar $H$

$$
S=\int \mathrm{d}(Z-w-\bar{w})\left[\frac{1}{2} M(H) \dot{H}^{2}-V(H)\right],
$$

where

$$
M(H)=\frac{\left(e^{2 H}+1\right)^{2}}{\left(e^{2 H}+1-\alpha^{2}\right)^{3 / 2}}, \quad V(H)=-\frac{\gamma^{2}}{2 \ell^{2}} \frac{e^{2 H}+1-2 \alpha^{2}}{\left(e^{2 H}+1-\alpha^{2}\right)^{1 / 2}} .
$$


Thus it is possible to use the energy conservation law of that model in order to evaluate the "velocity" $\dot{H}$ in terms of $H$. Since $\mathrm{d} H=\dot{H} \mathrm{~d}(Z-w-\bar{w})$ one has

$$
\frac{\mathrm{d}}{\mathrm{d} H}\left(\frac{1}{2} M(H) \dot{H}^{2}+V(H)\right)=0
$$

so that there must exist a constant $E$ such that

$$
\dot{H}=\sqrt{\frac{2}{M(H)}[E-V(H)]}=\sqrt{2} \frac{\left(e^{2 H}+1-\alpha^{2}\right)^{3 / 4}}{e^{2 H}+1}\left[E+\frac{\gamma^{2}}{2 \ell^{2}} \frac{e^{2 H}+1-2 \alpha^{2}}{\sqrt{e^{2 H}+1-\alpha^{2}}}\right]^{1 / 2} .
$$

The key-point is to consider now, as a new coordinate, the function $H$ in place of $w+\bar{w}^{13}$ and to write down the full solution, say metric plus gauge field, in terms of $H$. Using $w=x+i y$, the general solution is given by

$$
\begin{aligned}
\mathrm{d} s^{2} & =-4 \rho^{2} e^{2 \gamma Z / \ell}\left[\mathrm{d} t+e^{-\gamma Z / \ell} \hat{\sigma}_{y} \mathrm{~d} y\right]^{2}+\frac{e^{2 H}}{4 \rho^{2}} \mathrm{~d} y^{2}+\frac{1}{4 \rho^{2}}\left[\mathrm{~d} Z^{2}+e^{2 H}\left(\mathrm{~d} Z-\frac{\mathrm{d} H}{\dot{H}}\right)^{2}\right] \\
\mathcal{A} & =\ell \dot{H}\left[-2 i \rho^{2} \chi e^{\gamma Z / \ell} \mathrm{d} t+\left(1-e^{2 H} \chi^{2}\right) \mathrm{d} y\right]-i \frac{\ell}{4} \mathrm{~d} \log \frac{b}{\bar{b}}
\end{aligned}
$$

where $\dot{H}$ is given in equation (4.56), the functions $\chi$ and $\rho$ are defined in (4.51) and the shift vector reads

$$
\hat{\sigma}_{y}=-\frac{i}{2} e^{2 H} \frac{\chi}{\rho^{2}}
$$

If $\gamma=1$, a simple example of this set of solutions can be obtained by setting $\alpha=0$, SO

$$
\dot{H}=1 / \ell, \quad b=\frac{1}{2}\left(1+\frac{z}{\ell}\right)
$$

As will be shown in section 5.1.2, this corresponds to the maximally supersymmetric $\mathrm{AdS}_{4}$ solution. More general $\gamma=1$ solutions will be two-parameter deformations thereof, the parameters being $\alpha$ and the energy $E$ of the associated scalar system.

Setting $\gamma=0$ the potential $V(H)$ vanishes and the parameter $E$ can be fixed by a simple rescaling of the coordinates. Thus we are left with a one-parameter family of solutions. Since the metric does no more depend explicitly on $Z$, it is useful to replace the coordinate $Z$ instead of $x$ by $H$. Defining a new coordinate $r$ such that

\footnotetext{
${ }^{13}$ This is possible by simply requiring that $\dot{H} \neq 0$.
} 
$r^{4} \equiv 16\left(e^{2 H}+1-\alpha^{2}\right)$ and a new parameter $Q=\frac{4}{\ell} \alpha$, the complete solution reads

$$
\begin{aligned}
\mathrm{d} s^{2}= & -\left(\frac{r^{2}}{\ell^{2}}+\frac{Q^{2}}{r^{2}}\right)\left[\mathrm{d} t-\frac{2 \ell^{3} Q}{r^{4}+\ell^{2} Q^{2}} \mathrm{~d} y\right]^{2}+ \\
& +\left(\frac{r^{2}}{\ell^{2}}+\frac{Q^{2}}{r^{2}}\right)^{-1}\left[h(r)^{2}\left(\mathrm{~d} r+\frac{2}{h(r)} \mathrm{d} x\right)^{2}+\frac{1}{4}\left(r^{4}+\ell^{2} Q^{2}-16\right)\left(\mathrm{d} x^{2}+\mathrm{d} y^{2}\right)\right] \\
\mathcal{A}= & -\frac{Q}{r} \mathrm{~d} t+\frac{2 \ell}{r} \mathrm{~d} y-i \frac{\ell}{4} \mathrm{~d} \log \frac{b}{\bar{b}}
\end{aligned}
$$

where

$$
h(r)=\frac{r^{4}+\ell^{2} Q^{2}}{r^{4}+\ell^{2} Q^{2}-16} .
$$

The parameter $Q$ can thus be interpreted as an electric charge. The Petrov type of the solution is $D$ or simpler. If one sets $Q=4 / \ell$ the Petrov type is reduced to $N$, so that there is a gravitational wave.

In order to complete the classification of $G_{0}=0$ solutions, we need to study separately the cases where either $\psi_{1}$ or $\psi_{-}$vanishes (it can easily be seen from (4.39) and (4.40) that there is no solution if both vanish). As one can see by looking at equations (4.38) and (4.41), the condition $\psi_{1}=0$ leads to $b=b(z)$, which is studied in detail in section 5.1. The other possibility, $\psi_{-}=0$, is more involved, but as we show in appendix $\mathrm{D}$ it boils down to three different cases, that can be completely solved: the $\mathrm{AdS}_{2} \times \mathbb{H}^{2}$ anti-Nariai spacetime studied in section 5.1.1, the imaginary $b$ case solved in section 5.3, and finally the half BPS solution coming from the gravitational Chern-Simons model, that we analyse in section 5.5.

We would like to remark that the assumption $G_{0}=0$ on the overall time-dependence of the second Killing spinor seems a reasonable choice since all known 1/2-supersymmetric solutions to be studied in the next section are contained in this class, or can be brought to this class by a general coordinate transformation. Hence we expect the $G_{0}=0$ class to form an important subclass of all 1/2-supersymmetric solutions.

\section{Timelike half-supersymmetric examples}

The problem of finding all half BPS configurations in the timelike class involves the solution of the integrability conditions we obtained above. To obtain explicit examples of half BPS solutions, we shall restrict to some simple subclasses with particular $b$. This will determine the fraction of preserved supersymmetry for the solutions which are already known to be $1 / 4$ supersymmetric, and will also lead to new solutions. 


\subsection{Static Killing spinors and $b=b(z)$}

The timelike vector field $V$, constructed as a bilinear of the Killing spinor, is static if the associated one-form $V=\mathrm{d} t+\sigma$ satisfies the Fröbenius condition $V \wedge \mathrm{d} V=0$. Obviously, there can be static BPS solutions with $V$ not being static itself, due to the choice of coordinates; we shall loosely refer the Killing spinors whose vector bilinear is static as static Killing spinors. The staticity condition, in turn, implies $\mathrm{d} \sigma=0$ and puts strong constraints on the function $b$. Indeed, equation (4.18) implies that the phase $\varphi$ of $b$ depends only on $z$. Then, (4.19) gives the modulus $r$ of $b$ in terms of its phase,

$$
r=\frac{\sin \varphi(z)}{l \varphi^{\prime}(z)} .
$$

As a consequence, $r$ and therefore the complete complex function $b$, depend on the single variable $z$. The full solution is therefore determined by the single real function $\varphi$, which has to satisfy the equations for supersymmetry (together with the conformal factor $\psi$ ).

However, since the equations can be exactly solved for arbitrary $b(z)$, we will stick to this more general case and eventually comment on the static subcase.

If $b$ depends only on $z$, the equations of motion simplify to

$$
\begin{gathered}
\operatorname{Im}\left(b^{2} \partial_{z}^{2} \frac{1}{b}-\frac{3 b}{l} \partial_{z} \frac{1}{b}+\frac{1}{b l^{2}}\right)=0 \\
e^{-2 \xi} \Delta \xi=\frac{2}{l} e^{2 \Phi}\left[\partial_{z}\left(\frac{1}{b}+\frac{1}{\bar{b}}\right)-\frac{1}{l}\left(\frac{1}{b}+\frac{1}{\bar{b}}\right)^{2}+\frac{3}{l b \bar{b}}\right] .
\end{gathered}
$$

Here we have used the fact that $\Phi$, defined in (4.15), depends only on the coordinate $z$. In principle there is also an integration constant $K(w, \bar{w})$ with arbitrary dependence on the transverse coordinates, but since $\Phi$ appears only in the combination $\Phi+\xi$ in all the equations, we can always absorb the $(w, \bar{w})$ dependence into the conformal factor $\xi$. Now the left hand side of equation (5.3) depends only on the coordinates $w$ and $\bar{w}$, while the right hand side depends only on $z$. This equation can be therefore satisfied only if both sides are equal to some constant $\kappa$. The system of equations is then

$$
\begin{gathered}
\Delta \xi+\frac{\kappa}{2} e^{2 \xi}=0 \\
e^{2 \Phi(z)}\left[\partial_{z}\left(\frac{1}{b}+\frac{1}{\bar{b}}\right)-\frac{1}{l}\left(\frac{1}{b}+\frac{1}{\bar{b}}\right)^{2}+\frac{3}{l b \bar{b}}\right]=-\frac{l}{4} \kappa .
\end{gathered}
$$

Note that the first one is the Liouville equation, whose solution describes the transverse two-dimensional manifold, which has therefore constant curvature $\kappa$. 
Equations (5.2) and (5.5) can easily be solved [16]. Their solution is given by ${ }^{14}$

$$
\bar{b}=-\frac{\alpha z^{2}+\beta z+\gamma}{\ell(2 \alpha z+\beta)}
$$

with $\alpha, \beta, \gamma \in \mathbb{C}$. Then $\xi$ solves the Liouville equation for a constant curvature twomanifold with scalar curvature ${ }^{15}$

$$
\kappa=8(\alpha \bar{\gamma}+\bar{\alpha} \gamma)-4 \beta \bar{\beta} .
$$

This solution generically belongs to the supersymmetric Reissner-Nordström-TaubNUT-AdS 4 family of spacetimes. The values $\alpha=0$ and $\beta^{2}=4 \alpha \gamma$ are special cases and will be treated separately in the following. Note that the coefficients $\alpha, \beta$ and $\gamma$ are not three independent parameters, as they can be rescaled without changing the function $b$ : the solutions depend only on their ratios. For example, if $\alpha \neq 0$, one can use $\beta / \alpha$ and $\gamma / \alpha$ as independent complex parameters of the family of solutions.

The solutions with static Killing spinor form a subset of this family. For (5.6) the staticity condition (5.1) yields the condition $\alpha \bar{\beta}-\bar{\alpha} \beta=0$. Recalling the expression for the NUT charge of these solutions,

$$
n=\frac{i}{4}\left(\frac{\bar{\beta}}{\bar{\alpha}}-\frac{\beta}{\alpha}\right)
$$

this charge must vanish for non-vanishing $\alpha$, as one could have guessed. On the other hand, for $\alpha=0$ the solution is anti-Nariai, as we shall see below. We conclude that the most general supersymmetric configuration with static Killing vector constructed as a Killing spinor bilinear is either of the form (5.6) - i. e. in the fourth row of table 1 of [34] - with vanishing NUT charge, or it is anti-Nariai spacetime.

The supersymmetric static solutions discussed so far are generically 1/4-BPS. We want to see what further condition ensures the presence of an additional Killing spinor. Inserting the staticity ansatz $b=b(z)$ into the integrability equations and requiring these matrices to be of rank smaller or equal to two, one finds the following condition (in particular this is obtained from the vanishing of the minor of the last row of $\tilde{N}_{w t}$ and the first two rows of $\tilde{N}_{\bar{w} t}$ )

$$
\left(b^{\prime}+\frac{1}{l}\right)\left(2 b^{\prime}+\frac{1}{l}\right)\left[\partial_{z}\left(\frac{1}{b}+\frac{1}{\bar{b}}\right)-\frac{1}{l}\left(\frac{1}{b}+\frac{1}{\bar{b}}\right)^{2}+\frac{3}{l b \bar{b}}\right]=0,
$$

\footnotetext{
${ }^{14}$ With this definition, the constants $\alpha, \beta$ and $\gamma$ coincide with $a, b$ and $c$ of [16] respectively.

${ }^{15}$ This scalar curvature differs from the one given in [16] for the case in which all coefficients are real, $k=4 \alpha \gamma-\beta^{2}=\kappa / 4$. The factor of 4 comes from the different definition of the conformal factor of the transverse metric, our $\xi$ is related to the old $\gamma$ by $\xi=\gamma-\ln 2$.
} 
As an aside, note that we have only used the ansatz $b=b(z)$ so far and not the staticity condition (5.1), i.e. the precise relation between $r$ and $\varphi$. The static solutions are therefore in general still a subset of the solutions under consideration.

Condition (5.9) calls for the following three different cases, corresponding to the vanishing of its three factors.

\subsection{1 $\mathrm{AdS}_{2} \times \mathbb{H}^{2}$ space-time $(\alpha=0)$}

Requiring the first factor of (5.9) to vanish leads to $b=-\frac{z}{\ell}+i c$ with constant $c$, corresponding to $\alpha=0$ in (5.6). We can absorb the imaginary part of $c$ by a shift of the coordinate $z$ and henceforth will assume $c \in \mathbb{R}$.

In this case $\kappa=-4$ and we have a hyperbolic transverse space. As a solution of (5.4) we can take

$$
e^{2 \xi}=\frac{1}{2 x^{2}}
$$

Moreover, $e^{\Phi}=l|b|$ and $\sigma=0$, therefore giving the metric

$$
d s^{2}=-4\left(\frac{z^{2}}{\ell^{2}}+c^{2}\right) d t^{2}+\frac{d z^{2}}{4\left(\frac{z^{2}}{\ell^{2}}+c^{2}\right)}+\frac{\ell^{2}}{2 x^{2}}\left(d x^{2}+d y^{2}\right) .
$$

This is the anti-Nariai $\mathrm{AdS}_{2} \times \mathbb{H}^{2}$ solution, with the $\mathrm{AdS}_{2}$ factor written in Poincaré coordinates for $c=0$ and in global coordinates for $c \neq 0$. The coordinate transformations between Poincaré coordinates $\left(t_{P}, z_{P}\right)$ (with $\left.c=0\right)$ to global ones $\left(t_{g l}, z_{g l}\right)$ (with $c \neq 0)$ is given by

$$
\begin{aligned}
& z_{P}=\frac{1}{2 c}\left(z_{g l}-\sqrt{z_{g l}^{2}+\ell^{2} c^{2}} \cos \left(4 c t_{g l} / \ell\right)\right), \\
& t_{P}=-\frac{\ell}{2} \frac{\sqrt{z_{g l}^{2}+\ell^{2} c^{2}} \sin \left(4 c t_{g l} / \ell\right)}{z_{g l}-\sqrt{z_{g l}^{2}+\ell^{2} c^{2}} \cos \left(4 c t_{g l} / \ell\right)} .
\end{aligned}
$$

The electromagnetic field strength (4.21) in this case is given by

$$
\mathcal{F}=-\frac{1}{\ell x^{2}} d x \wedge d y
$$

i.e. only lives on the hyperbolic part and is independent of the coordinates of the AdS part of space-time.

This solution preserves precisely $1 / 2$ of the supersymmetries, as was already shown in [35]. To obtain the form of the Killing spinors admitted by this metric we first observe that the integrability conditions impose $\alpha_{2}=\alpha_{12}=0$. Then the Killing spinor equations are easily solved, but one should treat separately the cases $c=0$ and $c \neq 0$ : 
- If $c=0$, then

$$
\alpha_{0}=\lambda_{1}+\lambda_{2}\left(\frac{2 t}{\ell}-\frac{1}{2 b}\right), \quad \alpha_{1}=\frac{\lambda_{2}}{b},
$$

where $\lambda_{1,2} \in \mathbb{C}$ are integration constants. This yields the following Killing spinors, spanning a two-dimensional complex space,

$$
\epsilon=\left[\lambda_{1}+\lambda_{2}\left(\frac{2 t}{\ell}-\frac{1}{2 b}\right)\right] 1+b\left[\lambda_{1}+\lambda_{2}\left(\frac{2 t}{\ell}+\frac{1}{2 b}\right)\right] e_{2} .
$$

Note that $\lambda_{1}=1, \lambda_{2}=0$ corresponds to the original Killing spinor. Also note that the constant $G_{0}$, corresponding to the time-dependence of the second Killing spinor with $\lambda_{2} \neq 0$, is zero. The form of the scalar invariant corresponding to the general spinor $\epsilon$ is

$$
\tilde{b}=b\left[\left|\lambda_{1}\right|^{2}+\left|\lambda_{2}\right|^{2}\left(\frac{4 t^{2}}{\ell^{2}}-\frac{1}{4 b^{2}}\right)+\frac{2 t}{\ell}\left(\bar{\lambda}_{1} \lambda_{2}+\lambda_{1} \bar{\lambda}_{2}\right)\right]+\frac{1}{2}\left(\bar{\lambda}_{1} \lambda_{2}-\lambda_{1} \bar{\lambda}_{2}\right) .
$$

Here the first term is real, while the second is imaginary. Note that the latter is in fact constant. Then the Killing vector $\tilde{V}$ built from $\epsilon$ will have a norm $\tilde{V}^{2}=-4|\tilde{b}|^{2}$, and will be timelike unless $\tilde{b}$ vanishes. This is however not possible, because both the real and imaginary parts of $\tilde{b}$ should vanish, but since $\lambda_{1,2}$ do not depend on the coordinates, the real part cannot vanish. Therefore, every Killing spinor of this solution belongs to the timelike class.

- If $c \neq 0$ we have

$$
\alpha_{0}=\frac{1}{2 \sqrt{c}}\left[\lambda_{1}-i \lambda_{2}+\left(\lambda_{1}+i \lambda_{2}\right) \frac{b}{|b|} e^{-4 i c t / \ell}, \quad \alpha_{1}=-\frac{i \sqrt{c}}{|b|}\left(\lambda_{1}+i \lambda_{2}\right) e^{-4 i c t / \ell},\right.
$$

and the most general Killing spinor is parametrized by $\tilde{\lambda}_{1,2} \in \mathbb{C}$ as follows

$$
\epsilon=\frac{1}{2 \sqrt{c}}\left(\lambda_{1}-i \lambda_{2}\right)\left(1+b e_{2}\right)+\frac{b}{2|b|}\left(\lambda_{1}+i \lambda_{2}\right) e^{-4 i c t / \ell}\left(1+b^{*} e_{2}\right) .
$$

Note that the combination $\lambda_{1}-i \lambda_{2}$ corresponds to the first Killing spinor $1+b e_{2}$, while the orthogonal combination $\lambda_{1}+i \lambda_{2}$ gives rise to the second Killing spinor proportional to $1+b^{*} e_{2}$. Any combination with $\lambda_{2} \neq 0$ has $G_{0}=-4 i c / \ell$.

In this case, the real part of the invariant $\tilde{b}$ is given by

$$
\begin{aligned}
\operatorname{Re}(\tilde{b})= & \frac{\left|\lambda_{1}\right|^{2}}{2 \ell c}\left(-z+\sqrt{z^{2}+\ell^{2} c^{2}} \cos (4 c t / \ell)\right)+\frac{\left|\lambda_{2}\right|^{2}}{2 \ell c}\left(-z-\sqrt{z^{2}+\ell^{2} c^{2}} \cos (4 c t / \ell)\right)+ \\
& \left.+\frac{1}{2 \ell c}\left(\lambda_{1} \lambda_{2}^{*}+\lambda_{2} \lambda_{1}^{*}\right) \sqrt{z^{2}+\ell^{2} c^{2}} \sin (4 c t / \ell)\right),
\end{aligned}
$$

while the imaginary part is identical to that of (5.16). 
It can easily be checked that the coordinate transformation (5.12) indeed relates the complex scalar $\tilde{b}$, which is composed of spinor bilinears, in (5.16) and (5.19) to each other.

Let's now check how the isometries of $\mathrm{AdS}_{2}$ act on the Killing spinors. It is useful to do this by embedding $\mathrm{AdS}_{2}$ with metric

$$
d s^{2}=-4\left(\frac{z^{2}}{\ell^{2}}+c^{2}\right) d t^{2}+\frac{d z^{2}}{4\left(\frac{z^{2}}{\ell^{2}}+c^{2}\right)}
$$

into the three-dimensional flat space $X^{a}=(U, T, X)$ with metric

$$
d s^{2}=-d U^{2}-d T^{2}+d X^{2}
$$

Then, $\mathrm{AdS}_{2}$ is obtained as the hyperboloid defined by

$$
-U^{2}-T^{2}+X^{2}=\frac{\ell^{2}}{4}
$$

and its isometry group $\mathrm{SO}(2,1)$ will act as the three-dimensional Lorentz group on the embedding coordinates $X^{a}$ (here $a$ is a three-dimensional Lorentz index).

If $c=0$, the $\mathrm{AdS}_{2}$ metric (5.20) is in the Poincaré form, and can be seen to be the induced metric on the hyperboloid by parameterizing it with the coordinates $(t, z)$ given by

$$
z=U+X, \quad t=\frac{\ell T}{2(U+X)} .
$$

Then, if one defines the $3 \mathrm{~d}$ Lorentz vector

$$
\Lambda^{a}=\left(\frac{1}{\ell}\left(\left|\lambda_{1}\right|^{2}-\left|\lambda_{2}\right|^{2}\right), \frac{1}{\ell}\left(\lambda_{1}^{*} \lambda_{2}+\lambda_{1} \lambda_{2}^{*}\right),-\frac{1}{\ell}\left(\left|\lambda_{1}\right|^{2}+\left|\lambda_{2}\right|^{2}\right)\right),
$$

one explicitly checks that the invariant $\tilde{b}$ can be put in the form

$$
\tilde{b}=X_{a} \Lambda^{a}+\frac{i \ell}{2} \sqrt{\Lambda_{a} \Lambda^{a}}
$$

Now, the real and imaginary part of $\tilde{b}$ are independently manifestly invariant under the $\mathrm{AdS}_{2}$ isometries, as they should be (since they transform respectively as pseudoscalar and scalar under diffeomorphism $\left.{ }^{16}\right)$.

If $c \neq 0$ we have $\mathrm{AdS}_{2}$ in global coordinates, and the embedding is modified to

$$
U=-\frac{\ell}{2 c} \sqrt{\frac{z^{2}}{\ell^{2}}+c^{2}} \cos \frac{4 c t}{\ell}, \quad T=-\frac{\ell}{2 c} \sqrt{\frac{z^{2}}{\ell^{2}}+c^{2}} \sin \frac{4 c t}{\ell}, \quad X=\frac{z}{2 c} .
$$

\footnotetext{
${ }^{16}$ Note that $\Lambda$ doesn't depend on the sum of the phases of $\lambda_{1,2}$; this is diffeomorphism invariant but transforms under $\mathrm{U}(1)$ gauge transformations.
} 
The invariant (5.19) takes again the manifestly invariant form (5.25), as expected, and the isometries of $\mathrm{AdS}_{2}$ are realized linearly on the Killing spinors through their action on $\Lambda^{a}$.

This result may be useful to study in detail quotients of $\mathrm{AdS}_{2}$ and to see whether this operation breaks some supersymmetry.

\subsection{2 $\mathrm{AdS}_{4}$ space-time $\left(\beta^{2}=4 \alpha \gamma\right)$}

The following subcase corresponds to the vanishing of the second factor of the integrability condition (5.9). The function $b$ is then given by $b=-\frac{z}{2 l}+i c$, which can be obtained as the special case $\beta^{2}=4 \alpha \gamma$ from (5.6). This corresponds to $\mathrm{AdS}_{4}$, the only maximally supersymmetric solution of the theory. Indeed the integrability condition matrices vanish in this case.

Let's see in detail the form of the metric arising from different values of $c$. As in the previous case we can take the constant $c$ to be real. If $c=0$, the metric is static, $\sigma=0, \xi=0$ and $e^{2 \Phi}=|b|^{4}$, and we obtain anti-de Sitter in Poincaré coordinates,

$$
d s^{2}=-\frac{z^{2}}{\ell^{2}}\left(d t^{2}-d x^{2}-d y^{2}\right)+\frac{\ell^{2}}{z^{2}} d z^{2} .
$$

On the other hand, for $c \neq 0$, the metric appears in non-static coordinates,

$$
\sigma=-\frac{\ell d y}{4 c x^{2}}, \quad e^{2 \xi}=\frac{\ell^{2}}{4 c^{2} x^{2}}, \quad e^{2 \Phi}=|b|^{4}
$$

which give

$$
d s^{2}=-\left(\frac{z^{2}}{l^{2}}+4 c^{2}\right)\left[\left(d t-\frac{\ell d y}{4 c x^{2}}\right)^{2}-\frac{\ell^{2}}{16 c^{2} x^{2}}\left(d x^{2}+d y^{2}\right)\right]+\left(\frac{z^{2}}{l^{2}}+4 c^{2}\right)^{-1} d z^{2}
$$

The field strength (4.21) vanishes in this case.

We shall now obtain the form of the Killing spinors for $\mathrm{AdS}_{4}$, and will do this in the simpler $c=0$ case. The solution of the Killing spinor equations yields

$$
\begin{array}{llrl}
\alpha_{0} & =\lambda_{1}-\left(\frac{t}{\ell}+\frac{\ell}{z}\right) \lambda_{2}+\frac{\bar{w}}{\ell} \lambda_{3}, & \alpha_{2} & =-\frac{w z}{2 \ell^{2}} \lambda_{2}+\frac{1}{2}\left(1+\frac{z t}{\ell^{2}}\right) \lambda_{3}-\frac{z}{2 \ell} \lambda_{4}, \\
\alpha_{1} & =\frac{2 \ell}{z} \lambda_{2}, & \alpha_{12} & =\frac{w z}{2 \ell^{2}} \lambda_{2}+\frac{1}{2}\left(1-\frac{z t}{\ell^{2}}\right) \lambda_{3}+\frac{z}{2 \ell} \lambda_{4},
\end{array}
$$

where the coefficients $\lambda_{1, \ldots, 4}$ span a four dimensional complex space, as expected in the case of maximal supersymmetry. In the form basis of the spinors $\epsilon=c_{0} 1+c_{1} e_{1}+c_{2} e_{2}+$ 
$c_{12} e_{1} \wedge e_{2}$, we obtain

$$
\begin{array}{ll}
c_{0}=\lambda_{1}-\left(\frac{t}{\ell}+\frac{\ell}{z}\right) \lambda_{2}+\frac{\bar{w}}{\ell} \lambda_{3}, & c_{2}=-\frac{z}{2 \ell} \lambda_{1}+\frac{z}{2 \ell}\left(\frac{t}{\ell}-\frac{\ell}{z}\right) \lambda_{2}-\frac{z \bar{w}}{2 \ell^{2}} \lambda_{3}, \\
c_{1}=\frac{w}{\ell} \lambda_{2}-\left(\frac{t}{\ell}+\frac{\ell}{z}\right) \lambda_{3}+\lambda_{4}, & c_{12}=\frac{w z}{2 \ell^{2}} \lambda_{2}-\frac{z}{2 \ell}\left(\frac{t}{\ell}-\frac{\ell}{z}\right) \lambda_{3}+\frac{z}{2 \ell} \lambda_{4} .
\end{array}
$$

The new Killing spinors corresponding to $\lambda_{2}$ and $\lambda_{4}$ both have ${ }^{17} G_{0}=0$. To study the action of the $\mathrm{AdS}_{4}$ isometries it is useful to embed the hyperboloid in a five-dimensional flat space $(U, V, T, X, Y)$ with metric

$$
d s^{2}=-d U^{2}+d V^{2}-d T^{2}+d X^{2}+d Y^{2}
$$

Then, $\mathrm{AdS}_{4}$ is the hypersurface $-U^{2}+V^{2}-T^{2}+X^{2}+Y^{2}=-\ell^{2} / 4$ and its isometries are realized as the $\mathrm{SO}(3,2)$ isometries of the embedding space. The relation with the Poincaré coordinates is

$$
\frac{t}{\ell}=\frac{T}{U-V}, \quad \frac{x}{\ell}=\frac{X}{U-V}, \quad \frac{y}{\ell}=\frac{Y}{U-V}, \quad z=2(U-V) .
$$

If we define the vectors

$$
\ell \Lambda^{a}=\left(\begin{array}{c}
\left|\lambda_{1}\right|^{2}-\left|\lambda_{2}\right|^{2}+\left|\lambda_{3}\right|^{2}-\left|\lambda_{4}\right|^{2} \\
\left|\lambda_{1}\right|^{2}+\left|\lambda_{2}\right|^{2}-\left|\lambda_{3}\right|^{2}-\left|\lambda_{4}\right|^{2} \\
\lambda_{3} \bar{\lambda}_{4}+\bar{\lambda}_{3} \lambda_{4}-\bar{\lambda}_{1} \lambda_{2}-\lambda_{1} \bar{\lambda}_{2} \\
\lambda_{2} \bar{\lambda}_{4}+\bar{\lambda}_{2} \lambda_{4}-\bar{\lambda}_{1} \lambda_{3}-\lambda_{1} \bar{\lambda}_{3} \\
i\left(\lambda_{2} \bar{\lambda}_{4}-\bar{\lambda}_{2} \lambda_{4}+\bar{\lambda}_{1} \lambda_{3}-\lambda_{1} \bar{\lambda}_{3}\right)
\end{array}\right), \quad X^{a}=\left(\begin{array}{c}
U \\
V \\
T \\
X \\
Y
\end{array}\right)
$$

where the index $a=1, \ldots, 5$ is an $\mathrm{SO}(3,2)$ index raised and lowered using the metric (5.32), then

$$
\Lambda_{a} \Lambda^{a}=-\frac{1}{\ell^{2}}\left[\lambda_{3} \bar{\lambda}_{4}-\bar{\lambda}_{3} \lambda_{4}+\bar{\lambda}_{1} \lambda_{2}-\lambda_{1} \bar{\lambda}_{2}\right]^{2} \geq 0
$$

and the invariant $\tilde{b}$ for the Killing spinors reads

$$
\tilde{b}=c_{0}^{*} c_{2}+c_{1} c_{12}^{*}=X_{a} \Lambda^{a}+\frac{i \ell}{2} \sqrt{\Lambda_{a} \Lambda^{a}} .
$$

\footnotetext{
${ }^{17}$ Note that this does not hold for $\lambda_{3}$, whose time-dependence is not of the form derived in section 4.4. There is no contradiction however, since all solutions in this class have $P=0$ and hence are treated separately in appendix C. It is interesting to find that nevertheless the time-dependence of many Killing spinors in this class have the canonical $G_{0}$ time-dependence.
} 
This form of $\tilde{b}$ is manifestly invariant under the $\mathrm{AdS}_{4}$ isometries, and shows that under $\Lambda^{a}$ transforms in the fundamental representation of $\mathrm{SO}(3,2)$ under these transformations. Note that it has precisely the same form (5.25) as in the anti-Nariai case. Again, the explicit knowledge of the $\mathrm{AdS}_{4}$ isometry group action on the Killing spinors is important to study the supersymmetry of its quotients.

\subsubsection{The Reissner-Nordström-Taub-NUT-AdS family $_{4}$}

The last subcase corresponds to the vanishing of the third factor of the integrability condition (5.9). Note that this is precisely the expression in square brackets of equation (5.5) and the condition reads simply $\kappa=0$. Then $\xi$ is an harmonic function and the transverse space is flat. In particular, the solution (5.6) admits a second Killing spinor if

$$
|\beta|^{2}=2(\alpha \bar{\gamma}+\bar{\alpha} \gamma)
$$

Since $\alpha \neq 0$ we can define $\zeta=\operatorname{Im}(\beta / \alpha)$ and $\delta=\operatorname{Im}(\gamma / \alpha)$. Moreover, all equations are invariant under rigid translations in the $z$ directions, since the coordinate $z$ never appears explicitly in them. One can use this freedom to eliminate the real part of $\beta / \alpha$ by performing the redefinition $z \mapsto z-\frac{1}{2} \operatorname{Re}(\beta / \alpha)$. Hence this complete family of $1 / 2$ BPS solutions is determined by two real parameters $\zeta$ and $\delta$,

$$
b=-\frac{1}{\ell} \frac{z^{2}-i \zeta z+\frac{1}{4} \zeta^{2}-i \delta}{2 z-i \zeta} .
$$

Then $\sigma=-2 \zeta(r / \ell)^{2} d \vartheta$ and the resulting metric is

$$
\begin{aligned}
d s^{2}= & -\frac{\left(z^{2}+\frac{\zeta^{2}}{4}\right)^{2}+(\zeta z+\delta)^{2}}{\ell^{2}\left(z^{2}+\frac{\zeta^{2}}{4}\right)}\left(d t-\frac{2 \zeta}{\ell^{2}} r^{2} d \vartheta\right)^{2} \\
& +\frac{\ell^{2}\left(z^{2}+\frac{\zeta^{2}}{4}\right) d z^{2}}{\left(z^{2}+\frac{\zeta^{2}}{4}\right)^{2}+(\zeta z+\delta)^{2}}+\frac{4}{\ell^{2}}\left(z^{2}+\frac{\zeta^{2}}{4}\right)\left(d r^{2}+r^{2} d \vartheta^{2}\right)
\end{aligned}
$$

where we used polar coordinates $(r, \vartheta)$ in the $(w, \bar{w})$ plane. The charges of the solution are

$$
M=-\frac{\delta \zeta}{\ell^{2}}, \quad n=\frac{\zeta}{2}, \quad P=-\frac{\zeta^{2}}{2 \ell}, \quad Q=-\frac{\delta}{\ell} .
$$

Essentially, the imaginary part of $\gamma$ gives the electric charge and the imaginary part of $\beta$ determines the NUT charge. Note that the quantization condition $P=-\left(k \ell^{2}+4 n^{2}\right) / 2 \ell$ is also satisfied. In terms of the charges, the solution is given by

$$
b=-\frac{1}{\ell} \frac{(z-i n)^{2}+2 n^{2}+i \ell Q}{2(z-i n)} .
$$


The subfamily of static half BPS configurations is obtained by imposing the staticity condition $\zeta=0$ or equivalently vanishing NUT charge. It is parameterized by the single parameter left, $\delta \in \mathbb{R}$ and the solutions are restricted to have the following charges

$$
M=0, \quad n=0, \quad P=0, \quad Q=-\frac{\delta}{\ell} .
$$

In terms of the charges, the solution is given by

$$
b=-\frac{1}{\ell} \frac{z^{2}+i \ell Q}{2 z} .
$$

The metric and electromagnetic field strength for this solution read

$$
d s^{2}=-\left(\frac{Q^{2}}{z^{2}}+\frac{z^{2}}{\ell^{2}}\right) d t^{2}+\frac{d z^{2}}{\frac{Q^{2}}{z^{2}}+\frac{z^{2}}{\ell^{2}}}+4 \ell^{2} z^{2} d w d \bar{w}
$$

and

$$
\mathcal{F}=-\frac{Q}{z^{2}} \mathrm{~d} t \wedge \mathrm{d} z
$$

This is simply the backreacted $\mathrm{AdS}_{4}$ filled with the electric field generated by an electric charge $Q$ placed in its center $\zeta=0$. The solution has a singularity there. Note that this solution was already shown to be $1 / 2$ supersymmetric in [36]. It was also shown there that the Killing spinors are preserved if one compactifies the transverse two-dimensional plane to a two-torus.

We will now discuss the Killing spinors for these metrics. The integrability conditions impose $\alpha_{2}=0$ and

$$
\left(b^{\prime}+\frac{1}{\ell}-\frac{b}{\ell \bar{b}}\right) \alpha_{3}=\left(b^{\prime}+\frac{1}{\ell}\right) \alpha_{4} .
$$

With these constraints, the Killing spinor equations simplify, and can be solved to give

$$
\begin{gathered}
\alpha_{0}=\lambda_{1}+2 i \zeta \bar{w} \lambda_{2}, \quad \alpha_{1}=0 \\
\alpha_{2}=\frac{z^{2}+i \zeta z+\frac{\zeta^{2}}{4}+i \delta}{\sqrt{4 z^{2}+\zeta^{2}}}, \quad \alpha_{12}=\alpha_{2}-\frac{\lambda_{2}}{2} \sqrt{4 z^{2}+\zeta^{2}}
\end{gathered}
$$

where $\lambda_{1,2} \in \mathbb{C}$ parameterize the two dimensional space of Killing spinors. Then the most general Killing spinor for these metrics is

$$
\begin{aligned}
\epsilon=( & \left.\lambda_{1}+2 i \zeta \bar{w} \lambda_{2}\right) 1-\ell \lambda_{2} \sqrt{\frac{2 z+i \zeta}{2 z-i \zeta}} e_{1} \\
& +b\left(\lambda_{1}+2 i \zeta \bar{w} \lambda_{2}\right) e_{2}-\frac{z^{2}-i \zeta z+\frac{\zeta^{2}}{4}-i \delta}{\sqrt{4 z^{2}+\zeta^{2}}} \lambda_{2} e_{1} \wedge e_{2} .
\end{aligned}
$$


Again the second Killing spinor has $G_{0}=0$ time-dependence. Finally, the corresponding orbit of the Killing spinor is determined by the invariant

$$
\tilde{b}=b\left|\lambda_{1}\right|^{2}+\left(\ell \frac{z^{2}+i \zeta z+\frac{\zeta^{2}}{4}+i \delta}{2 z-i \zeta}+4 \zeta^{2} b w \bar{w}\right)\left|\lambda_{2}\right|^{2}+2 i \zeta b\left(\bar{w} \bar{\lambda}_{1} \lambda_{2}-w \lambda_{1} \bar{\lambda}_{2}\right) .
$$

It is easy to show now that $\tilde{b}$ is non vanishing for any choice of $\lambda_{1,2}$ : indeed if $\tilde{b}=0$, we have $\partial \tilde{\partial} \tilde{b}=4 \zeta^{2} b\left|\lambda_{2}\right|^{2}=0$ and either $\lambda_{2}=0$, which implies in turn $\lambda_{1}=0$, or $\zeta=0$. In the latter case, it is very easy to see that $\tilde{b}=0$ iff $\epsilon=0$. Therefore, all Killing spinors of this family of metrics belong to the timelike class, and the solution is purely timelike.

Summary of the $b=b(z)$ case:

1. The only supersymmetric solutions with static Killing spinor (i.e. whose timelike Killing vector constructed as a Killing spinor bilinear is static) are $\mathrm{AdS}_{4}$, the anti-Nariai spacetime and the Reissner-Nordström-AdS 4 solutions of the fourth row of table 1 of [34], i. e. solutions of the form (5.6) with vanishing NUT charge.

2. The only $1 / 2$ BPS solutions with static Killing spinor are the anti-Nariai spacetime and the solution (5.43) with field strength (5.44).

3. The most general half BPS solution with $b=b(z)$ are the anti-Nariai spacetime and the solution (5.39) with charges (5.40) describing an electric charge in the center of $\mathrm{AdS}_{4}$.

The natural way to continue this approach is to study half BPS solutions with $b$ harmonic, and this will be the subject of the next paragraph.

\subsection{Harmonic $b$ solutions}

The previous class of solutions can be generalized by requiring $\Delta b=0$ instead of $b=b(z)[16]$. This implies that $\Delta 1 / b=0$ and hence (4.23) still simplifies in exactly the same way as in the $b=b(z)$ case. Indeed, the solution is

$$
\bar{b}=-\frac{\alpha z^{2}+\beta z+\gamma}{\ell(2 \alpha z+\beta)}
$$

where now $\alpha, \beta$ and $\gamma$ are no more constants but arbitrary functions of $(w, \bar{w})$. It is then easy to show that the $\Delta b=0$ condition requires these functions to be harmonic and all (anti-)holomorphic, that is $\alpha, \beta$ and $\gamma$ all depending either only on $w$ or only on $\bar{w}$, and this is the most general solution with $\Delta b=0$. The $b=b(z)$ configurations 
are particular cases of this larger class, and are obtained for $\alpha, \beta$ and $\gamma$ constant. Note that also the $\partial b=0$ and $\partial \bar{b}=0$ subclasses fall into this family.

Let's take for definiteness $\alpha, \beta, \gamma$ all anti-holomorphic, then $b=b(z, w)$. The requirement that the integrability conditions allow for an extra Killing spinor, i.e. that they are of rank $\leq 2$, in this case leads to several conditions. One of these is obtained from the minor of the last three lines of $\tilde{N}_{w t}$ and reads

$$
\left(2 \partial_{z} \bar{b}+\frac{1}{l}\right)\left(\partial_{z} \bar{b}+\frac{1}{l}\right)\left(\partial^{2} b+\frac{1}{b} \partial b \partial b-2 \partial(\Phi+\xi) \partial b\right) \partial b=0 .
$$

This gives three different cases to be analysed, corresponding to the vanishing of the first three factors of this equation (vanishing of the fourth factor implies $b=b(z)$ and hence brings one back to the previous section).

\subsubsection{Deformations of $\mathrm{AdS}_{2} \times \mathbb{H}^{2}$}

The vanishing of the first factor in (5.51) implies $b=-\frac{z}{\ell}+i c(w)$, where $c(w)$ is an arbitrary holomorphic function. These are the $\alpha(w)=0$ supersymmetric Kundt solutions of Petrov type II, describing gravitational and electro-magnetic waves propagating on anti-Nariai space-time [16].

The remaining integrability conditions however imply $\alpha_{1}=\alpha_{2}=\alpha_{12}=0$, in which case there is no second Killing spinor, or $\partial c=0$. Therefore there are no new half BPS solutions with non constant $c$. In this class $c$ constant is the half supersymmetric anti-Nariai spacetime and the other preserve only $1 / 4$ of the supersymmetries.

\subsubsection{Deformations of $\mathrm{AdS}_{4}$}

The vanishing of the second factor in (5.51) implies $b=-\frac{z}{2 \ell}+i c(w)$. In this case we are considering the $\beta^{2}=4 \alpha \gamma$ supersymmetric Kundt solutions, describing gravitational and electro-magnetic waves propagating on $\mathrm{AdS}_{4}$ spacetime [16].

Again the remaining integrability equations have to solutions: $\alpha_{1}=\alpha_{2}=\alpha_{12}=0$ or $\partial c=0$. Hence, as in the previous case, we find that there are no harmonic deformations of $\mathrm{AdS}_{4}$ preserving half supersymmetry.

\subsubsection{Deformations of Reissner-Nordström-Taub-NUT-AdS 4}

Not considering the previous two special cases, the general solution represents expanding gravitational and electro-magnetic waves propagating on a Reissner-NordströmTaub-NUT-AdS ${ }_{4}$ spacetime [16]. When $\operatorname{Im}(\beta)=0$, the solution can be put in RobinsonTrautman form and is of Petrov type II.

The vanishing of the third factor in (5.51) is given by

$$
\partial^{2} b+\frac{1}{b} \partial b \partial b-2 \partial(\Phi+\xi) \partial b=0 .
$$


With $b$ given in (5.50) this case can be solved for the derivative of $\Phi+\xi$ and implies

$$
\bar{\partial}(\Phi+\xi)=\frac{1}{2 \partial b}\left(\partial^{2} b+\frac{1}{b} \partial b \partial b\right),
$$

and therefore $\Delta(\Phi+\xi)=0$. Then (5.3) fixes the transverse manifold to be flat and

$$
\kappa(w)=8(\alpha \bar{\gamma}+\bar{\alpha} \gamma)-4 \beta \bar{\beta}=0 .
$$

But $\alpha, \beta$ and $\gamma$ being holomorphic, this last equation can be satisfied if and only if they are constant, and we are back to the previous case, i. e. there are no new $1 / 2$ BPS solutions.

\section{Summary of the harmonic case:}

There are no new half BPS solutions in the harmonic $b$ case. The only half BPS solutions are those with $b=b(z)$, and as soon as one deforms these solutions by adding some harmonic $(w, \bar{w})$-dependence, one breaks supersymmetry further to $1 / 4$.

\subsection{Imaginary $b$ solutions}

Another subcase we want to study is $\bar{b}=-b$, i. e. $b$ purely imaginary. For notational convenience we introduce ${ }^{18}$

$$
b=i X,
$$

where $X$ is real. From (4.15) one gets $\Phi=0$. All quantities in the Bianchi identity (4.22), apart from $b$ and hence $X$, are then $z$-independent. The only consistent possibility is to take $\partial_{z} X=0$. The remaining equations (4.22) and (4.23) read

$$
\Delta \xi=\frac{6}{\ell^{2} X^{2}} e^{2 \xi}, \quad \Delta \frac{1}{X}-\frac{4}{\ell^{2} X^{3}} e^{2 \xi}=0 .
$$

Examples of $1 / 4$ supersymmetric solutions of this class, i.e. with imaginary $b$, that were discussed in [16] are $X=(x / \ell)^{\alpha}$ with $\alpha=-2$ and $\alpha=\frac{1}{3}$. These correspond to a particular Petrov type I solution and an electrovac AdS travelling wave of Petrov type $\mathrm{N}$, respectively. It was shown that the latter actually preserves a second, null Killing spinor. In this section we will derive the general condition for $1 / 2$ supersymmetry in the case of imaginary $b$ and will find that there is a one-parameter family of such solutions.

The condition for $1 / 2$ supersymmetry is very simple in this case. Assuming that $\partial X$ is not equal to zero, which would clearly be incompatible with (5.55), there is

\footnotetext{
${ }^{18}$ In the following we will assume that $X$ is positive without loss of generality.
} 
only one differential constraint which needs to be satisfied for the existence of a second Killing spinor, i. e. for the matrices of integrability conditions to have rank 2, namely

$$
\partial^{2} X^{-1}-2 \partial \xi \partial X^{-1}=0
$$

The above three differential equations can be integrated to

$$
e^{2 \xi}=-i \bar{K}(\bar{w}) \partial X^{-1}, \quad \partial X^{-1}=\frac{i}{\ell^{2}} K(w)\left(\frac{1}{4 X^{4}}+L\right),
$$

where $K(w)$ is an arbitrary holomorphic function and $L$ is a real constant. The function $K(w)$ corresponds to the freedom to choose holomorphic coordinates on the twodimensional space, and hence it can be gauged away. A convenient gauge choice will be $K(w)=i \ell$. Note that, for this choice, the imaginary part of the right hand side of the last equation vanishes, and therefore that $\partial_{y} X=0$.

For $L=0$, 5.57) can be integrated to give

$$
X^{3}=\frac{3 x}{2 \ell},
$$

which is (up to a rescaling of the coordinate $x$ ) the example given above with $\alpha=\frac{1}{3}$. This was already found to be $1 / 2$ supersymmetric in [16]. Here we find that this solution is a special case of the most general possibility.

For other values of the constant $L$ it is convenient to use $X$ as a new coordinate instead of solving for $X(x)$. From (4.18) and (4.19) it follows that $\sigma$ can be chosen to be

$$
\sigma=\frac{\mathrm{d} y}{4 X^{4}}
$$

Then the metric reads

$$
d s^{2}=-4 X^{2}\left(d t+\frac{d y}{4 X^{4}}\right)^{2}+\frac{1}{4 X^{2}} d z^{2}+\frac{\ell^{2} d X^{2}}{X^{2}\left(1+4 L X^{4}\right)}+\frac{1+4 L X^{4}}{4 X^{6}} d y^{2}
$$

Finally, from (4.21) we obtain the gauge field strength

$$
\mathcal{F}=2 \mathrm{~d} t \wedge \mathrm{d} X
$$

Note that the geometry $(5.60)$ is generically of Petrov type D, and becomes of Petrov type $\mathrm{N}$ for $L=0$.

Now let us turn our attention to the form of the second Killing spinor. First of all, the integrability conditions imply that it takes the form

$$
\alpha^{T}=\left(\beta_{1}, \beta_{2}, i X^{3} e^{\xi} \beta_{2}, i X^{3} e^{\xi} \beta_{2}\right),
$$


where $\beta_{1}$ and $\beta_{2}$ are arbitrary space-time dependent functions. The Killing spinor equations (4.31) yield

$$
\beta_{1}=\lambda_{1}-\frac{1}{2} \lambda_{2} b^{-2}, \quad \beta_{2}=\lambda_{2} b^{-2}
$$

where $\lambda_{1}$ and $\lambda_{2}$ are integration constants. This implies that the new Killing spinor takes the form $\epsilon=\lambda_{1} \epsilon_{1}+\lambda_{2} \epsilon_{2}$, where

$$
\epsilon_{1}=1+i X e_{2}, \quad \epsilon_{2}=\frac{1}{2} X^{-2}\left(1-i X e_{2}\right)+\sqrt{\frac{1}{4} X^{-4}+L}\left(e_{1}-i X e_{1} \wedge e_{2}\right) .
$$

Note that $G_{0}=0$ as well in this class.

One interesting aspect of the second Killing spinor $\epsilon_{2}$ is the norm of its associated Killing vector $V_{\mu}=D\left(\epsilon_{2}, \Gamma_{\mu} \epsilon_{2}\right)$. We find $V_{\mu} V^{\mu}=-4 X^{2} L^{2}$, hence the second Killing spinor is indeed null for the case $L=0$, as was noticed before, while it is timelike for $L \neq 0$. In the latter case, to understand whether the solution belongs also to the null class of supersymmetric solutions, we have therefore to study the most general linear combination of the two Killing spinors. The Killing vector $\tilde{V}$ constructed from $\epsilon=\lambda_{1} \epsilon_{1}+\lambda_{2} \epsilon_{2}$ has norm

$$
\tilde{V}^{2}=\frac{1}{X^{2}}\left(\bar{\lambda}_{1} \lambda_{2}-\lambda_{1} \bar{\lambda}_{2}\right)^{2}-4 X^{2}\left(L\left|\lambda_{1}\right|^{2}+\left|\lambda_{2}\right|^{2}\right)^{2}
$$

which can vanish only if $L \leq 0$. We have therefore three cases:

1. $L>0$, pure timelike class, Petrov type D.

2. $L=0$, belongs to both null and timelike classes, Petrov type N. This is the homogeneous half BPS pp-wave in AdS. (In the terminology of [16] it has a wave profile $\mathcal{G}_{\alpha}$ with $\alpha=0$ ).

3. $L<0$, belongs to both null and timelike classes, Petrov type D.

Actually the solutions (5.60) with $L>0$ can be cast into a simpler form. This is done by trading the coordinate $y$ for a new variable $\psi=L y-t$. For convenience, let us also introduce the Schwarzschild coordinate $r$ and rescale $z$,

$$
r=-\frac{\ell}{\sqrt{L} X}, \quad \zeta=\frac{1}{2} \sqrt{L} z
$$

In the new coordinates, the metric and the gauge field strength read

$$
d s^{2}=-\left(\frac{r^{2}}{\ell^{2}}+\frac{q_{e}^{2}}{r^{2}}\right) d t^{2}+\frac{d r^{2}}{\frac{r^{2}}{\ell^{2}}+\frac{q_{e}^{2}}{r^{2}}}+\frac{r^{2}}{\ell^{2}}\left(d \psi^{2}+d \zeta^{2}\right), \quad \mathcal{F}=\frac{q_{e}}{r^{2}} d t \wedge d r
$$


where we have defined $q_{e}=2 \ell / \sqrt{L}$. This is precisely the half BPS solution obtained in [36], the massless limit of an electrically charged toroidal black hole, which forms a naked singularity. It is also interesting to note that the charge $q_{e}$ diverges in the $L \rightarrow 0$ limit. This limit is naively singular in these coordinates, but it can be taken if we perform a Penrose limit $[37,38]$. The existence of this limit explains why we obtained a one-parameter family of geometries (5.60) connecting the massless limit of toroidal black holes and a pp-wave. Indeed, define the new coordinates $\left(X^{+}, X^{-}, R, Z\right)$ and the rescaled charge $Q_{e}$ by

$$
\psi+t=2 \epsilon^{2} X^{+}, \quad \psi-t=2 X^{-}, \quad r=\frac{1}{\epsilon R}, \quad \zeta=\epsilon Z, \quad q_{e}=\frac{Q_{e}}{\epsilon} .
$$

Then, the singular limit $\epsilon \rightarrow 0$ yields is a regular solution of the theory and corresponds to the half supersymmetric solution (5.60) with $L=0$,

$$
d s^{2}=\frac{\ell^{2}}{R^{2}}\left(4 d X^{+} d X^{-}-\frac{Q_{e}^{2} R^{4}}{\ell^{6}} d X^{-2}+d R^{2}+d Z^{2}\right), \quad \mathcal{F}=\frac{Q_{e}}{\ell^{2}} \mathrm{~d} X^{-} \wedge \mathrm{d} R .
$$

In the procedure, we have blown up the metric in the neighborhood of a geodesic with $\psi+t$ constant near the boundary $r \rightarrow \infty$ of AdS.

We now turn to the $L<0$ case, which is both timelike and lightlike. Let us define $L=-\mu^{2}$. We can perform a coordinate transformation inspired from the previous one,

$$
\psi=L y-t, \quad r=-\frac{\ell}{\mu X}, \quad \zeta=\frac{\mu}{2} z,
$$

under which the metric and the field strength become

$$
d s^{2}=\left(\frac{r^{2}}{\ell^{2}}-\frac{q_{e}^{2}}{r^{2}}\right) d t^{2}+\frac{d r^{2}}{\frac{r^{2}}{\ell^{2}}-\frac{q_{e}^{2}}{r^{2}}}+\frac{r^{2}}{\ell^{2}}\left(-d \psi^{2}+d \zeta^{2}\right), \quad \mathcal{F}=\frac{q_{e}}{r^{2}} d t \wedge d r
$$

where we have defined $q_{e}=2 \ell / \mu$. We see that this is the precisely the metric for $L>0$ after the double analytic continuation

$$
t \mapsto i t, \quad \psi \mapsto i \psi, \quad q_{e} \mapsto-i q_{e}
$$

This solution represents therefore a bubble of nothing in AdS [39-42]. Note that the metric is singular for $r=\sqrt{\ell q_{e}}$. One should compactify $t$, in such a way to eliminate the conical singularity on the $(t, r)$ hypersurface. Then, if we compactify also $\zeta$, this $S^{1}$ will have a minimal radius for $r=\sqrt{\ell q_{e}}$ (the boundary of the bubble of nothing) and then grow with $r$. Note that for $r \rightarrow \infty$ one locally recovers AdS spacetime, and that the $L=0$ solutions can again be understood as a Penrose limit of this metric. 


\subsection{Action of the $\operatorname{PSL}(2, \mathbb{R})$ group on the imaginary $b$ solutions}

We can now generate new supersymmetric solutions by acting with the $\operatorname{PSL}(2, \mathbb{R})$ symmetry group (4.28)-(1.29) on the known ones. It is easy to check that the $\mathrm{AdS}_{4}$ and $\mathrm{AdS}_{2} \times \mathbb{H}^{2}$ solutions are invariant under this group (although it acts non trivially on the Killing spinors). Its action on the $b=b(z)$ subfamily of the RNTN-AdS 4 solutions was studied in [16], where it was shown that it acts non trivially on the charges, by mixing them. Here we want to apply it to the imaginary $b$ solutions of the previous paragraph.

The new solution solution of the supersymmetry equations (4.22)-(4.23) generated by the transformation (4.28)-(4.29) is

$$
\tilde{b}=-\frac{\gamma^{2} X z^{2}}{2 \gamma^{2} \ell X z+i}, \quad e^{2(\tilde{\Phi}+\xi)}=\frac{\gamma^{4} z^{4}}{4 X^{4}}\left(1+4 L X^{4}\right),
$$

where, without loss of generality, we eliminated $\alpha$ by means of a translation of $z^{19}$, and dropped the prime of the new coordinate $z^{\prime}$. The shift function is then determined by solving equations (4.18) and (4.19),

$$
\sigma_{x}=0, \quad \sigma_{y}=\frac{1+4 L X^{4}}{4 \gamma^{2} X^{4} z^{2}}+\frac{\gamma^{2} \ell^{2}}{X^{2}} .
$$

Then, defining the new coordinates $(T, \sigma, p, q)$ through

$$
T=\frac{t}{2 \ell^{2} \gamma^{2}}, \quad \sigma=\frac{y}{2}, \quad p=-\frac{\ell}{X}, \quad q=2 \ell^{2} \gamma^{2} z,
$$

the metric reads

$$
\begin{array}{r}
d s^{2}=-\frac{Q(q)}{q^{2}+p^{2}}\left[d T+\left(\frac{P(p)}{q^{2}}+\frac{p^{2}}{\ell^{2}}\right) d \sigma\right]^{2}+\frac{q^{2}+p^{2}}{Q(q)} d q^{2}+\frac{q^{2}+p^{2}}{P(p)} d p^{2} \\
+\frac{1}{\ell^{4}}\left(q^{2}+p^{2}\right) P(p) d \sigma^{2}
\end{array}
$$

with

$$
Q(q)=\frac{q^{4}}{\ell^{2}}, \quad P(p)=\frac{1}{\ell^{2}}\left(p^{4}+4 L \ell^{2}\right),
$$

and the gauge field (4.21) is

$$
\mathcal{F}=\mathrm{d}\left(\frac{p q^{2}}{\ell\left(q^{2}+p^{2}\right)}\right) \wedge \mathrm{d} T+\mathrm{d}\left(\frac{4 \ell L p}{q^{2}+p^{2}}\right) \wedge \mathrm{d} \sigma .
$$

\footnotetext{
${ }^{19}$ After this translation the limit $\gamma \rightarrow 0$ is not anymore well-defined. To perform it, one has to substitute preliminarily $z$ with $z-\alpha / \gamma$ everywhere.
} 
The form of the metric suggests some connection with the Plebanski-Demianski family of solutions, and indeed these geometries are of Petrov type D for $L \neq 0$, and of Petrov type $\mathrm{N}$ for $L=0$, but we were not able to find the precise relation. Note also that the parameter $\gamma$ has been reabsorbed in the new variables, and we are left with a one-parameter $(L)$ family of solutions.

The left hand side of the necessary condition (4.34) for the existence of a second Killing spinor reads, for this solution,

$$
-\frac{9 i X^{4}\left(1+4 L X^{4}\right)}{\ell^{2}\left(1+4 \gamma^{4} \ell^{2} X^{2} z^{2}\right)^{4}} \gamma^{2}
$$

which clearly vanishes only for $\gamma=0$, i.e. if the $\operatorname{PSL}(2, \mathbb{R})$ transformation is trivial. Therefore, the new solutions (5.73)-(5.75) preserves only $1 / 4$ of the supersymmetries, and we explicitly see that the $\operatorname{PSL}(2, \mathbb{R})$ transformations can break any additional supersymmetry. Also note that if we perform the $\operatorname{PSL}(2, \mathbb{R})$ transformation adapting the original metric to a different Killing spinor, we could in principle end up with other supersymmetric solutions.

Surprisingly, we find that the $L=0$ solution can be cast in the Lobatchevski wave form, even though it only has a time-like Killing spinor. This can be seen by trading the coordinates $(q, p)$ for $(x, z)$ defined by

$$
x=\frac{\ell^{3}}{2}\left(\frac{1}{q^{2}}-\frac{1}{p^{2}}\right), \quad z=\frac{\ell^{3}}{q p},
$$

in the metric (5.73) with $L=0$, which becomes

$$
d s^{2}=\frac{\ell^{2}}{z^{2}}\left(-2 d T d \sigma+\frac{z^{2}}{2 \ell \sqrt{x^{2}+z^{2}}} \frac{x-\sqrt{x^{2}+z^{2}}}{x+\sqrt{x^{2}+z^{2}}} d T^{2}+d z^{2}+d x^{2}\right) .
$$

The field strength can be easily obtained from equation (5.75) but the result is not particularly enlightening and therefore we do not report it. This metric represents a 1/4 BPS Lobatchevski wave, whose Killing spinor falls in the timelike class. This does

not contradict the results obtained in the null case, since the null Lobatchevski had a field strength (3.6) of the form $\mathcal{F}=\phi^{\prime}(T) \mathrm{d} T \wedge \mathrm{d} z$, while this solution has a much more complicated gauge field. It is however interesting to note that the solutions of the null case do not exhaust all possible supersymmetric Lobatchevski waves.

\subsection{Gravitational Chern-Simons system and $G_{0}=\psi_{-}=0$ solutions}

A number of the previously studied subcases can be combined into the interesting Ansatz

$$
b=-\frac{1}{\ell} \frac{\alpha z^{2}+\beta z+\gamma}{2 \alpha z+\beta-i \eta(w, \bar{w})},
$$


where $\alpha, \beta$ and $\gamma$ are three real constants. For $\alpha=\beta=0$ this reduces to $b$ imaginary, while $\eta=0$ leads to the real subcase of $b=b(z)$. With this assumption, the equations for a timelike Killing spinor reduce to

$$
\Delta \xi+\frac{1}{2} e^{2 \xi}(k-3 \eta)=0, \quad \Delta \eta+e^{2 \xi}\left(k \eta-\eta^{3}\right)=0
$$

where we have defined $k=4 \alpha \gamma-\beta^{2}$ and $\Delta=4 \partial \bar{\partial}$. Interestingly, as shown in [16], this system of equations follows from the dimensionally reduced Chern-Simons action $[43,44]$,

$$
S=\int d^{2} x \sqrt{{ }^{(2)} g}\left({ }^{(2)} R \eta+\eta^{3}\right)
$$

if we use the conformal gauge ${ }^{(2)} g_{i j} d x^{i} d x^{j}=e^{2 \xi}\left(d x^{2}+d y^{2}\right)$ and $\eta$ is the curl of a vector potential, $\sqrt{{ }^{(2)} g} \epsilon_{i j} \eta=\partial_{i} A_{j}-\partial_{j} A_{i}$. To obtain equations (5.80) we vary the action with respect to $A_{i}$ and $\xi$. When varying the dimensionally reduced Chern-Simons action with respect to $g_{i j}$ there is however an additional equation to (5.80).

Using the results of Grumiller and Kummer [48], one obtains the most general solution to the dimensionally reduced Chern-Simons system [16]

$$
e^{2 \xi}=\frac{L}{\ell^{4}}-\frac{k}{2} \eta^{2}+\frac{1}{4} \eta^{4}
$$

where $L$ is an integration constant and $\mathrm{d} \eta=e^{2 \xi} \mathrm{d} x$. Trading the coordinate $x$ for $\eta$, we get the following configuration of the fields

$$
\begin{aligned}
\mathrm{d} s^{2} & =-\frac{4}{\ell^{2}} \frac{P_{2}^{2}}{P_{2}^{\prime 2}+\eta^{2}}[\mathrm{~d} t+\sigma]^{2}+\frac{\ell^{2}}{4} \frac{P_{2}^{\prime 2}+\eta^{2}}{P_{2}^{2}}\left[\mathrm{~d} z^{2}+P_{2}^{2}\left(e^{-2 \xi} \mathrm{d} \eta^{2}+e^{2 \xi} \mathrm{d} y^{2}\right)\right], \\
\mathcal{A} & =\frac{2}{\ell} \frac{P_{2} \eta}{P_{2}^{\prime 2}+\eta^{2}}[\mathrm{~d} t+\sigma]+\frac{\ell}{4} \mathcal{V} \mathrm{d} y-i \frac{\ell}{4} \mathrm{~d} \log \frac{b}{\bar{b}},
\end{aligned}
$$

where $P_{2}(z)=\alpha z^{2}+\beta z+\gamma, k$ is defined as above and the shift function reads

$$
\sigma=\frac{\ell^{2}}{2}\left(\alpha \eta^{2}+\frac{e^{2 \xi}}{P_{2}}\right) \mathrm{d} y
$$

These solutions preserve $1 / 4$ of the original supersymmetry. In fact, the $k=0$ solutions coincide with the imaginary $b$ ones and their $\operatorname{PSL}(2, \mathbb{R})$ transforms of sections 5.3 and 5.4. For $k$ non-vanishing these are different solutions.

As can be seen from the Poisson bracket (4.34), the only possibility to have $1 / 2$ supersymmetry is $\alpha=0$ and hence $k \leq 0$. In fact, starting from any solution with $k \leq 0$, one can always obtain $\alpha=0$ by an appropriate $\operatorname{PSL}(2, \mathbb{R})$ transformation. The 
non-trivial part of the $\operatorname{PSL}(2, \mathbb{R})$ symmetry is $z \mapsto-1 /(z+\delta)$, whose action on the parameters $\alpha, \beta$ and $\gamma$ of the Ansatz (5.79) is given by

$$
\alpha \mapsto \alpha \delta^{2}-\beta \delta+\gamma, \quad \beta \mapsto 2 \alpha \delta-\beta, \quad \gamma \mapsto \alpha,
$$

which keeps $k$ fixed. Indeed, for $k \leq 0$, there is always a $\operatorname{PSL}(2, \mathbb{R})$ transformation that sets $\alpha=0$, while this is impossible for $k>0$.

The requirement $\alpha=0$ leads to the half-supersymmetric imaginary $b$ solution of section 5.3 for $k=0$. In the case of $k$ negative, when $\alpha=0$ one can scale $\beta$ to 1 in

(5.79) without loss of generality, and $\gamma$ can be put to zero by a translation in $z$. Hence the function $b$ is given by

$$
b=-\frac{1}{\ell} \frac{z}{1-i \eta} .
$$

The metric is given in (D.32) and is generically of Petrov type $D$. The second Killing spinor can be found in (D.33). As shown in appendix $\mathrm{D}$, the $G_{0}=\psi_{-}=0$ solutions are either the imaginary $b$ ones, anti-Nariai spacetime or the above $1 / 2$ supersymmetric solution with $k=-1$.

We would like to mention that (5.82) is the most general solution to the dimensionally reduced Chern-Simons system, but not to the equations (5.80). The reason for this is the additional constraint one obtains when varying (5.81) with respect to $g_{i j}$. An example of this is provided by the Petrov type I solution with $b=i(x / \ell)^{2}$ in section 5.3 and its $\operatorname{PSL}(2, \mathbb{R})$ transform given in eq. (2.44) of [16].

\section{Final remarks}

In this paper, we applied spinorial geometry techniques to classify all supersymmetric solutions of minimal $\mathcal{N}=2$ gauged supergravity in four dimensions.

In the presence of null Killing spinors, the problem can be completely solved, and all 1/4- and 1/2-supersymmetric solutions have been written down explicitly. We showed that there are no $1 / 4$-BPS backgrounds with $U(1) \ltimes \mathbb{R}^{2}$-invariant Killing spinors and those with $\mathbb{R}^{2}$-invariant Killing spinors have been derived in sections 3.1 and 3.2. The backgrounds in the latter section were previously unknown and are Petrov type II configurations describing gravitational waves propagating on a bubble of nothing in $\mathrm{AdS}_{4}$. In addition, it turned out that there are no $1 / 2$-BPS backgrounds with $\mathbb{R}^{2}$ invariant Killing spinors and hence any additional Killing spinor is timelike. In section 3.3 we gave the backgrounds with one null and one timelike Killing spinor.

For a timelike Killing spinor we derived the conditions for the corresponding backgrounds in section 4.1 and 4.2. We worked out the first integrability conditions necessary for the existence of a second Killing spinor in section 4.3. We explicitly solved 
these equations in a number of subcases in section 5, and thereby found several new solutions, like the bubbles of nothing in $\mathrm{AdS}_{4}$, already obtained in the null formalism, and their PSL $(2, \mathbb{R})$-transformed configurations. Furthermore, our results showed that the generalized holonomy in the case of one preserved complex supercharge is contained in $\mathrm{A}(3, \mathbb{C})$, supporting thus the classification scheme of [4].

In addition, the time-dependence of a second time-like Killing spinor was shown to be an overall exponential factor with coefficient $G_{0}$ in section 4.4 . In the case $G_{0}=0$ these equations have been solved in full generality, up to a second order ordinary differential equation. We expect this class to comprise a large number of interesting 1/2-BPS solutions. Indeed, all the examples of section 5 either have vanishing $G_{0}$ or can be transformed to that case by a coordinate transformation.

There are several interesting points that remain to be understood. First of all, it would be desirable to get a deeper insight into the underlying geometric structure in the case of $\mathrm{U}(1)$ invariant spinors. In five dimensions, spacetime is a fibration over a four-dimensional Hyperkähler or Kähler base for ungauged and gauged supergravity respectively $[8,12]$, whereas in four-dimensional ungauged supergravity one has a fibration over a three-dimensional flat space [5]. This suggests that the base for $D=4$ gauged supergravity might be an odd-dimensional analogue of a Kähler manifold, i. e. , a Sasaki manifold. From the equations (4.22) and (4.23) this is not obvious.

Secondly, in [16], a surprising relationship between the equations (4.22), 4.23) governing 1/4 BPS solutions and the gravitational Chern-Simons theory [43] was found. Why such a relationship should exist is not clear at all, and deserves further investigations.

The third point concerns preons, which were conjectured in [45] to be elementary constituents of other BPS states. In type II and eleven-dimensional supergravity, it was shown that imposing 31 supersymmetries implies that the solution is locally maximally supersymmetric $[27,30,46]$. Similar results in four- and five-dimensional gauged supergravity were obtained in $[28,29]$. This implies that preonic backgrounds are necessarily quotients of maximally supersymmetric solutions. While M-theory preons cannot arise by quotients [47], it remains to be seen if $3 / 4$ supersymmetric solutions to $\mathcal{N}=2$, $D=4$ or $D=5$ gauged supergravities really do not exist. The only maximally supersymmetric backgrounds in these theories are $\mathrm{AdS}_{4}$ [13] and $\mathrm{AdS}_{5}$ [12] respectively, so the putative preonic configurations must be quotients of AdS.

Finally, it would be interesting to apply spinorial geometry techniques to classify all supersymmetric solutions of four-dimensional $\mathcal{N}=2$ matter-coupled gauged supergravity. Work in this direction is in progress [49]. 


\section{Acknowledgments}

We are grateful to Alessio Celi, Marcello Ortaggio and Christoph Sieg for useful discussions. This work was partially supported by INFN, MURST and by the European Commission program MRTN-CT-2004-005104. D.R. wishes to thank the Università di Milano for hospitality. Part of this work was completed while he was a post-doc at King's College London, for which he would like to acknowledge the PPARC grant $\mathrm{PPA} / \mathrm{G} / \mathrm{O} / 2002 / 00475$. In addition, he is presently supported by the European ECRTN project MRTN-CT-2004-005104, MCYT FPA 2004-04582-C02-01 and CIRIT GC 2005SGR-00564.

\section{A. Spinors and forms}

In this appendix, we summarize the essential information needed to realize the spinors of $\operatorname{Spin}(3,1)$ in terms of forms. For more details, we refer to [50]. Let $V=\mathbb{R}^{3,1}$ be a real vector space equipped with the Lorentzian inner product $\langle\cdot, \cdot\rangle$. Introduce an orthonormal basis $e_{1}, e_{2}, e_{3}, e_{0}$, where $e_{0}$ is along the time direction, and consider the subspace $U$ spanned by the first two basis vectors $e_{1}, e_{2}$. The space of Dirac spinors is $\Delta_{c}=\Lambda^{*}(U \otimes \mathbb{C})$, with basis $1, e_{1}, e_{2}, e_{12}=e_{1} \wedge e_{2}$. The gamma matrices are represented on $\Delta_{c}$ as

$$
\begin{aligned}
& \left.\left.\Gamma_{0} \eta=-e_{2} \wedge \eta+e_{2}\right\rfloor \eta, \quad \Gamma_{1} \eta=e_{1} \wedge \eta+e_{1}\right\rfloor \eta, \\
& \left.\left.\Gamma_{2} \eta=e_{2} \wedge \eta+e_{2}\right\rfloor \eta, \quad \Gamma_{3} \eta=i e_{1} \wedge \eta-i e_{1}\right\rfloor \eta,
\end{aligned}
$$

where

$$
\eta=\frac{1}{k !} \eta_{j_{1} \ldots j_{k}} e_{j_{1}} \wedge \ldots \wedge e_{j_{k}}
$$

is a $k$-form and

$$
e_{i} \wedge \eta=\frac{1}{(k-1) !} \eta_{i j_{1} \ldots j_{k-1}} e_{j_{1}} \wedge \ldots \wedge e_{j_{k-1}} .
$$

One easily checks that this representation of the gamma matrices satisfies the Clifford algebra relations $\left\{\Gamma_{a}, \Gamma_{b}\right\}=2 \eta_{a b}$. The parity matrix is defined by $\Gamma_{5}=i \Gamma_{0} \Gamma_{1} \Gamma_{2} \Gamma_{3}$, and one finds that the even forms $1, e_{12}$ have positive chirality, $\Gamma_{5} \eta=\eta$, while the odd forms $e_{1}, e_{2}$ have negative chirality, $\Gamma_{5} \eta=-\eta$, so that $\Delta_{c}$ decomposes into two complex chiral Weyl representations $\Delta_{c}^{+}=\Lambda^{\text {even }}(U \otimes \mathbb{C})$ and $\Delta_{c}^{-}=\Lambda^{\text {odd }}(U \otimes \mathbb{C})$.

Let us define the auxiliary inner product

$$
\left\langle\sum_{i=1}^{2} \alpha_{i} e_{i}, \sum_{j=1}^{2} \beta_{j} e_{j}\right\rangle=\sum_{i=1}^{2} \alpha_{i}^{*} \beta_{i}
$$


on $U \otimes \mathbb{C}$, and then extend it to $\Delta_{c}$. The $\operatorname{Spin}(3,1)$ invariant Dirac inner product is then given by

$$
D(\eta, \theta)=\left\langle\Gamma_{0} \eta, \theta\right\rangle
$$

In many applications it is convenient to use a basis in which the gamma matrices act like creation and annihilation operators, given by

$$
\begin{aligned}
\left.\Gamma_{+} \eta \equiv \frac{1}{\sqrt{2}}\left(\Gamma_{2}+\Gamma_{0}\right) \eta=\sqrt{2} e_{2}\right\rfloor \eta, & \Gamma_{-} \eta \equiv \frac{1}{\sqrt{2}}\left(\Gamma_{2}-\Gamma_{0}\right) \eta=\sqrt{2} e_{2} \wedge \eta \\
\Gamma \bullet \eta \equiv \frac{1}{\sqrt{2}}\left(\Gamma_{1}-i \Gamma_{3}\right) \eta=\sqrt{2} e_{1} \wedge \eta, & \left.\Gamma_{\bullet} \eta \equiv \frac{1}{\sqrt{2}}\left(\Gamma_{1}+i \Gamma_{3}\right) \eta=\sqrt{2} e_{1}\right\rfloor \eta .
\end{aligned}
$$

The Clifford algebra relations in this basis are $\left\{\Gamma_{A}, \Gamma_{B}\right\}=2 \eta_{A B}$, where $A, B, \ldots=$ $+,-, \bullet, \bar{\bullet}$ and the nonvanishing components of the tangent space metric read $\eta_{+-}=$ $\eta_{-+}=\eta_{\bullet \bullet}=\eta_{\boldsymbol{\bullet} \bullet}=1$. The spinor 1 is a Clifford vacuum, $\Gamma_{+} 1=\Gamma_{\boldsymbol{\bullet}} 1=0$, and the representation $\Delta_{c}$ can be constructed by acting on 1 with the creation operators $\Gamma^{+}=\Gamma_{-}, \Gamma^{\bar{\bullet}}=\Gamma_{\bullet}$, so that any spinor can be written as

$$
\eta=\sum_{k=0}^{2} \frac{1}{k !} \phi_{\bar{a}_{1} \ldots \bar{a}_{k}} \Gamma^{\bar{a}_{1} \ldots \bar{a}_{k}} 1, \quad \bar{a}=+, \bar{\bullet} .
$$

The action of the Gamma matrices and the Lorentz generators $\Gamma_{A B}$ is summarized in the table 6 .

\begin{tabular}{|c||c|c|c|c|}
\hline & 1 & $e_{1}$ & $e_{2}$ & $e_{1} \wedge e_{2}$ \\
\hline \hline$\Gamma_{+}$ & 0 & 0 & $\sqrt{2}$ & $-\sqrt{2} e_{1}$ \\
\hline$\Gamma_{-}$ & $\sqrt{2} e_{2}$ & $-\sqrt{2} e_{1} \wedge e_{2}$ & 0 & 0 \\
\hline$\Gamma_{\bullet}$ & $\sqrt{2} e_{1}$ & 0 & $\sqrt{2} e_{1} \wedge e_{2}$ & 0 \\
\hline$\Gamma_{\boldsymbol{\bullet}}$ & 0 & $\sqrt{2}$ & 0 & $\sqrt{2} e_{2}$ \\
\hline \hline$\Gamma_{+-}$ & 1 & $e_{1}$ & $-e_{2}$ & $-e_{1} \wedge e_{2}$ \\
\hline$\Gamma_{\boldsymbol{\bullet} \bullet}$ & 1 & $-e_{1}$ & $e_{2}$ & $-e_{1} \wedge e_{2}$ \\
\hline$\Gamma_{+\bullet}$ & 0 & 0 & $-2 e_{1}$ & 0 \\
\hline$\Gamma_{+\boldsymbol{\bullet}}$ & 0 & 0 & 0 & 2 \\
\hline$\Gamma_{-\bullet}$ & $-2 e_{1} \wedge e_{2}$ & 0 & 0 & 0 \\
\hline$\Gamma_{-\mathbf{\bullet}}$ & 0 & $2 e_{2}$ & 0 & 0 \\
\hline
\end{tabular}

Table 6: The action of the Gamma matrices and the Lorentz generators $\Gamma_{A B}$ on the different basis elements. 
Note that $\Gamma_{A}=U_{A}{ }^{a} \Gamma_{a}$, with

$$
\left(U_{A}{ }^{a}\right)=\frac{1}{\sqrt{2}}\left(\begin{array}{cccc}
1 & 0 & 1 & 0 \\
-1 & 0 & 1 & 0 \\
0 & 1 & 0 & -i \\
0 & 1 & 0 & i
\end{array}\right) \in \mathrm{U}(4)
$$

so that the new tetrad is given by $E^{A}=\left(U^{*}\right)^{A}{ }_{a} E^{a}$.

\section{B. Spinor bilinears}

Given a Killing spinor

$$
\epsilon=c_{0} 1+c_{1} e_{1}+c_{2} e_{2}+c_{12} e_{1} \wedge e_{2},
$$

one can construct the bilinears

$$
\begin{aligned}
& \tilde{f}=-i D(\epsilon, \epsilon)=-i\left(c_{0} c_{2}^{*}-c_{1} c_{3}^{*}-c_{2} c_{0}^{*}+c_{12} c_{1}^{*}\right), \\
& \tilde{g}=-i D\left(\epsilon, \Gamma_{5} \epsilon\right)=c_{0} c_{2}^{*}+c_{1} c_{3}^{*}+c_{2} c_{0}^{*}+c_{12} c_{1}^{*} \text {, } \\
& \tilde{V}=D\left(\epsilon, \Gamma_{\mu} \epsilon\right) \mathrm{d} x^{\mu}=\frac{1}{2}\left[\frac{1}{|b|^{2}}\left(\left|c_{2}\right|^{2}+\left|c_{12}\right|^{2}\right)-\left|c_{0}\right|^{2}-\left|c_{1}\right|^{2}\right] \mathrm{d} z \\
& -2\left[\left|c_{2}\right|^{2}+\left|c_{12}\right|^{2}+|b|^{2}\left(\left|c_{0}\right|^{2}+\left|c_{1}\right|^{2}\right)\right](\mathrm{d} t+\sigma) \\
& +\frac{1}{|b|} e^{\psi}\left[\left(c_{2} c_{1}^{*}-c_{0} c_{12}^{*}\right) \mathrm{d} w+\left(c_{1} c_{2}^{*}-c_{12} c_{0}^{*}\right) \mathrm{d} \bar{w}\right], \\
& \tilde{B}=D\left(\epsilon, \Gamma_{5} \Gamma_{\mu} \epsilon\right) \mathrm{d} x^{\mu}=\frac{1}{2}\left[\frac{1}{|b|^{2}}\left(\left|c_{2}\right|^{2}-\left|c_{12}\right|^{2}\right)+\left|c_{0}\right|^{2}-\left|c_{1}\right|^{2}\right] \mathrm{d} z \\
& -2\left[\left|c_{2}\right|^{2}-\left|c_{12}\right|^{2}-|b|^{2}\left(\left|c_{0}\right|^{2}-\left|c_{1}\right|^{2}\right)\right](\mathrm{d} t+\sigma) \\
& +\frac{1}{|b|} e^{\psi}\left[\left(c_{2} c_{1}^{*}+c_{0} c_{12}^{*}\right) \mathrm{d} w+\left(c_{1} c_{2}^{*}+c_{12} c_{0}^{*}\right) \mathrm{d} \bar{w}\right], \\
& \tilde{\Phi}=\frac{1}{2} D\left(\epsilon, \Gamma_{\mu \nu} \epsilon\right) \mathrm{d} x^{\mu} \wedge \mathrm{d} x^{\nu}=-\left(c_{0} c_{2}^{*}-c_{1} c_{12}^{*}+c_{2} c_{0}^{*}-c_{12} c_{1}^{*}\right) \mathrm{d} t \wedge \mathrm{d} z \\
& -\frac{2 e^{\psi}}{|b|}\left(c_{2} c_{12}^{*}+|b|^{2} c_{0} c_{1}^{*}\right) \mathrm{d} t \wedge \mathrm{d} w-\frac{2 e^{\psi}}{|b|}\left(c_{12} c_{0}^{*}+4|b|^{2} c_{1} c_{0}^{*}\right) \mathrm{d} t \wedge \mathrm{d} \bar{w} \\
& +\left[\left(c_{0} c_{2}^{*}-c_{1} c_{12}^{*}+c_{2} c_{0}^{*}-c_{12} c_{1}^{*}\right) \sigma_{w}+\frac{e^{\psi}}{2|b|^{3}} c_{2} c_{12}^{*}-\frac{e^{\psi}}{2|b|} c_{0} c_{1}^{*}\right] \mathrm{d} z \wedge \mathrm{d} w \\
& +\left[\left(c_{0} c_{2}^{*}-c_{1} c_{12}^{*}+c_{2} c_{0}^{*}-c_{12} c_{1}^{*}\right) \sigma_{\bar{w}}+\frac{e^{\psi}}{2|b|^{3}} c_{12} c_{0}^{*}-\frac{e^{\psi}}{2|b|} c_{1} c_{0}^{*}\right] \mathrm{d} z \wedge \mathrm{d} \bar{w}
\end{aligned}
$$




$$
\begin{aligned}
+\frac{2 e^{\psi}}{|b|}\left[c_{2} c_{12}^{*} \sigma_{\bar{w}}-\right. & c_{12} c_{0}^{*} \sigma_{w}+|b|^{2}\left(c_{0} c_{1}^{*} \sigma_{\bar{w}}-c_{1} c_{0}^{*} \sigma_{w}\right) \\
+ & \left.\frac{e^{\psi}}{4|b|}\left(c_{0} c_{2}^{*}+c_{1} c_{12}^{*}-c_{2} c_{0}^{*}-c_{12} c_{1}^{*}\right)\right] \mathrm{d} w \wedge \mathrm{d} \bar{w} .
\end{aligned}
$$

Given the first Killing spinor of the form $\epsilon_{1}=1+b e_{2}$ and the second Killing spinor $\epsilon_{2}=c_{0} 1+c_{1} e_{1}+c_{2} e_{2}+c_{12} e_{1} \wedge e_{2}$, one can also construct mixed bilinears of the type $D\left(\epsilon_{1}, \Gamma_{\ldots} \epsilon_{2}\right)$, which verify the same differential equations as the bilinears built from the original two Killing spinors:

$$
\begin{aligned}
& \hat{f}=-i\left(\bar{b} c_{0}-c_{2}\right), \quad \hat{g}=\bar{b} c_{0}+c_{2}, \\
& \hat{V}=\frac{1}{2 b}\left(c_{2}+b c_{0}\right)(\mathrm{d} t+\sigma)+\frac{1}{2 b}\left(c_{2}-b c_{0}\right) \mathrm{d} z+\frac{1}{|b|} e^{\psi}\left(\bar{b} c_{1}-c_{12}\right) \mathrm{d} \bar{w}, \\
& \hat{B}=\frac{1}{2 b}\left(c_{2}-b c_{0}\right)(\mathrm{d} t+\sigma)+\frac{1}{2 b}\left(c_{2}+b c_{0}\right) \mathrm{d} z+\frac{1}{|b|} e^{\psi}\left(\bar{b} c_{1}+c_{12}\right) \mathrm{d} \bar{w} .
\end{aligned}
$$

\section{The case $P^{\prime}=0$}

In section 4.3, we simplified the equations for the second Killing spinor under the assumption $P^{\prime} \neq 0$, where $P=e^{-2 \psi} b \partial b$. Here we consider the case $P^{\prime}=0$. To this end, we need the following subset of the Killing spinor equations (4.31):

$$
\begin{gathered}
\partial_{+} \psi_{2}-\sqrt{2} r^{2}\left(\frac{\bar{b}^{\prime}}{\bar{b}}+\frac{1}{\ell \bar{b}}\right) \psi_{2}-\sqrt{2} r^{2}\left(\frac{b^{\prime}}{b}+\frac{1}{\ell b}\right) \psi_{12}=0 \\
\partial_{+} \psi_{12}-r e^{-\psi} \partial_{\bullet} \ln \bar{b} \psi_{1}-\sqrt{2} r^{2}\left(2 \frac{r^{\prime}}{r}+\frac{1}{\ell \bar{b}}\right) \psi_{12}=0 \\
\partial_{-} \psi_{2}+\frac{1}{r} e^{-\psi} \partial_{\bullet} \ln b \psi_{1}-\sqrt{2}\left(2 \frac{r^{\prime}}{r}+\frac{1}{\ell b}\right) \psi_{2}=0 \\
\partial_{-} \psi_{12}-\sqrt{2}\left(\frac{\bar{b}^{\prime}}{\bar{b}}+\frac{1}{\ell \bar{b}}\right) \psi_{2}-\sqrt{2}\left(\frac{b^{\prime}}{b}+\frac{1}{\ell b}\right) \psi_{12}=0 \\
r e^{-\psi} \partial_{\bullet}\left(\frac{1}{r^{2}} e^{2 \psi} \psi_{2}\right)-\sqrt{2}\left(\frac{b^{\prime}}{b}+\frac{1}{\ell b}\right) \psi_{1}=0 \\
r e^{-\psi} \partial_{\bullet}\left(\frac{1}{r^{2}} e^{2 \psi} \psi_{12}\right)+\sqrt{2}\left(\frac{\bar{b}^{\prime}}{\bar{b}}+\frac{1}{\ell \bar{b}}\right) \psi_{1}=0
\end{gathered}
$$

If $P^{\prime}=0$, 4.35) implies $\psi_{-}=0$ or $\partial P=0$. Let us first assume the former, i. e. , $\psi_{2}=\psi_{12}$. From (C.6) - C.5 one obtains then $\psi_{1}=0$ or

$$
\frac{b^{\prime}}{b}+\frac{\bar{b}^{\prime}}{\bar{b}}+\frac{1}{\ell b}+\frac{1}{\ell \bar{b}}=0 .
$$


- If $\psi_{1}=0$, (C.4) - C.3) yields $\psi_{2}=0$, and thus there exists no further Killing spinor.

- If (C.7) holds, one can use (C.1) and (C.4) to show that $\partial_{+} \psi_{2}=\partial_{-} \psi_{2}=0$, or equivalently $\partial_{t} \psi_{2}=\psi_{2}^{\prime}=0$. Using this in (C.2) and (C.3) and deriving with respect to $t$, one gets $\bar{\partial} \bar{b} \partial_{t} \psi_{1}=\bar{\partial} b \partial_{t} \psi_{1}=0$. When $\partial_{t} \psi_{1} \neq 0$, this means that $\bar{\partial} b=\partial b=0$, so $b=b(z)$, which is a case analyzed in section 5.1. If instead $\partial_{t} \psi_{1}=0$, all the $\psi_{i}$ are independent of $t$, and the Killing spinor equations reduce to the system (4.38) to (4.49) with $G_{0}=0$.

In the case $\partial P=0$, consider the integrability condition

$$
\psi_{1} Q^{\prime}+\psi_{-} \partial Q=0,
$$

where $Q=e^{-2 \psi} \bar{b} \partial \bar{b}$, following from the first line of $\tilde{N}_{w t}$. As long as $Q^{\prime} \neq 0$, with the same reasoning as in section 4.3, one obtains the system (4.38) to (4.49). If $Q^{\prime}=0$, (C.8) implies $\psi_{-}=0$ or $\partial Q=0$. The case $\psi_{-}=0$ was already considered above, so the only remaining case is $P^{\prime}=\partial P=Q^{\prime}=\partial Q=0$. For $P=Q=0$ we get again $b=b(z)$, so without loss of generality we can assume $P \neq 0$ or $Q \neq 0$. Suppose that $Q=0, P \neq 0$, so $b=b(w, z)$. Take the logarithm of $e^{-2 \psi} b \partial b=P(\bar{w})$, derive with respect to $z$, use (4.15), and apply $\bar{\partial}$. This leads to $\partial b=0$, which is a contradiction to the assumption $P \neq 0$. In the same way one shows that $P=0, Q \neq 0$ is not possible, so that both $P$ and $Q$ must be nonvanishing. Now use the third row of $\tilde{N}_{\bar{w} t}$, which leads to $\bar{Q} \psi_{2}=0$ and hence $\psi_{2}=0$. Finally, the last row of $\tilde{N}_{\bar{w} t}$ yields $\psi_{-}=0$, i. e. , the case already considered above.

Hence, the conclusion is that in the case $P^{\prime}=0$, the second Killing spinor either has $G_{0}$ time-dependence of the form (4.37), or leads to solutions with $b=b(z)$. The latter are treated separately in section 5.1. As can be found there, all 1/2-BPS solutions with $b=b(z)$ also have second Killing spinors with $G_{0}$ time-dependence of the form (4.37). Hence this time-dependence is a completely general result ${ }^{20}$ for second Killing spinors in the time-like case.

\section{Half-supersymmetric solutions with $G_{0}=0$}

From the difference of equations (4.39) - (4.43) and (4.48) - (4.49) one gets $\psi_{-}=\psi_{-}(w)$. Furthermore, [4.42) $\left.-(4.40)+e^{-2 \psi}(4.47)\right]$ and (4.44) yield $\psi_{1}=\psi_{1}(z)$. Assuming $\psi_{-} \neq$

\footnotetext{
${ }^{20}$ The only counterexample is the third Killing spinor of $\mathrm{AdS}_{4}$, see (5.30), but since this is maximally supersymmetric it does not contradict the result.
} 
0 , eqns. (4.38) and (4.41) can be written in the form

$$
\left(\frac{\beta}{b}\right)^{\prime}+\partial\left(\frac{1}{b}\right)=0, \quad\left(\frac{\beta}{\bar{b}}\right)^{\prime}+\partial\left(\frac{1}{\bar{b}}\right)=0,
$$

where $\beta=\psi_{1} / \psi_{-}$. Deriving (D.1) with respect to $\bar{w}$ gives

$$
\left[\bar{\partial}\left(\frac{1}{b}\right) \beta\right]^{\prime}+\partial \bar{\partial}\left(\frac{1}{b}\right)=0, \quad\left[\bar{\partial}\left(\frac{1}{\bar{b}}\right) \beta\right]^{\prime}+\partial \bar{\partial}\left(\frac{1}{\bar{b}}\right)=0 .
$$

Now use (D.1) in the difference between the first equation and the complex conjugate of the second one to get

$$
\left[\frac{1}{b}\left(\bar{\beta} \beta^{\prime}-\bar{\beta}^{\prime} \beta\right)\right]^{\prime}=0 .
$$

Observe that $\bar{\beta} \beta^{\prime}-\bar{\beta}^{\prime} \beta=\left|\psi_{-}\right|^{-2}\left(\bar{\psi}_{1} \psi_{1}^{\prime}-\bar{\psi}_{1}^{\prime} \psi_{1}\right)(z)$, so that for $\bar{\psi}_{1} \psi_{1}^{\prime}-\bar{\psi}_{1}^{\prime} \psi_{1} \neq 0$ there must exist a real function $B(z)$ and a generic function $h(w, \bar{w})$ such that

$$
b=B(z) h(w, \bar{w}) .
$$

Plugging this into (D.1), we conclude that

$$
\partial \ln \left(\frac{h}{\bar{h}}\right)=0,
$$

so that the phase of the function $h$ is fixed, $h=h_{R}(w, \bar{w}) e^{i \varphi_{0}}$, with $h_{R}$ real. Using (4.18), the constancy of the phase of $b$ implies that the shift vector $\sigma$ does not depend on $z$. (4.19) gives then

$$
\partial_{z}\left(\frac{e^{2 \psi}}{B^{3}}\right)=0
$$

or, using (4.15),

$$
\frac{2}{3} \frac{\cos \varphi_{0}}{\ell h_{R}}+B^{\prime}=0
$$

and thus

$$
B^{\prime}=c, \quad \frac{2}{3} \frac{\cos \varphi_{0}}{\ell h_{R}}=-c,
$$

where $c$ denotes a real constant. Now we have to distinguish to cases:

1. $c \neq 0$ : In this case $b(z)=\left(B_{0}-\frac{2 \cos \varphi_{0}}{3 \ell} z\right) e^{i \varphi_{0}}$. Plugging this into the first of eqns. (D.1) one gets

$$
\left(\frac{\psi_{1}}{b}\right)^{\prime}=0
$$

which is solved by $\psi_{1}=\eta b$ where $\eta$ is a constant. But this yields $\bar{\psi}_{1} \psi_{1}^{\prime}-\bar{\psi}_{1}^{\prime} \psi_{1}=0$, which contradicts our assumption. 
2. $c=0$ : In this case $b(w, \bar{w})=i h_{R}(w, \bar{w})$. The combination 4.40$)+(4.42)-(4.39)-$ (4.43) leads to $\psi_{-}=0$, which again contradicts one of our assumptions.

We thus conclude that $\bar{\psi}_{1} \psi_{1}^{\prime}-\bar{\psi}_{1}^{\prime} \psi_{1}=0$, and hence $\psi_{1}=\zeta(z) e^{i \theta_{0}}$ where $\theta_{0}$ is a constant and $\zeta(z)$ is a real function. Sending $\psi_{i} \rightarrow e^{-i \theta_{0}} \psi_{i}$ we can take $\psi_{1}$ real and non-negative without loss of generality. Let us now consider the case where both $\psi_{1}$ and $\psi_{-}$are non-vanishing. This allows to introduce new coordinates $Z, W, \bar{W}$ such that

$$
\mathrm{d} Z=\frac{1}{\psi_{1}(z)} \mathrm{d} z, \quad \mathrm{~d} W=\frac{\mathrm{d} w}{\psi_{-}(w)}, \quad \mathrm{d} \bar{W}=\frac{\mathrm{d} \bar{w}}{\bar{\psi}_{-}(\bar{w})} .
$$

Note that one can set $\psi_{-}=1$ using the residual gauge invariance $w \mapsto W(w), \psi \mapsto$ $\tilde{\psi}=\psi-\frac{1}{2} \ln (\mathrm{d} W / \mathrm{d} w)-\frac{1}{2} \ln (\mathrm{d} \bar{W} / \mathrm{d} \bar{w})$ leaving invariant the metric $e^{2 \psi} \mathrm{d} w \mathrm{~d} \bar{w}$. We can thus take $W=w$ in the following. Equations (4.38) and (4.41) are then equivalent to

$$
\left(\partial_{Z}+\partial\right) \varphi=0, \quad \partial_{Z} \ln \psi_{1}-\left(\partial_{Z}+\partial\right) \ln r=0 \text {. }
$$

From the real part of the first equation we have

$$
\varphi=\varphi(Z-w-\bar{w}) .
$$

Using $\psi_{1}=\psi_{1}(Z)$, the second equation implies

$$
\left(\partial_{Z}+\partial\right) \frac{r}{\psi_{1}}=0
$$

and hence

$$
\frac{r}{\psi_{1}}=\rho(Z-w-\bar{w}) .
$$

The function $b$ must thus have the form

$$
b(Z, w+\bar{w})=\psi_{1}(Z) B(Z-w-\bar{w}),
$$

where $B(Z-w-\bar{w})=\rho(Z-w-\bar{w}) e^{i \varphi(Z-w-\bar{w})}$. The difference between (4.45) and (4.46) yields

$$
\left(\partial_{Z}+\partial\right)\left(\ln \psi_{1}-\psi\right)=0
$$

so that $\ln \psi_{1}-\psi=-H(Z-w-\bar{w})$ with $H$ real. This gives

$$
e^{2 \psi}=\psi_{1}(Z)^{2} e^{2 H}
$$

for the conformal factor. In terms of the new coordinate $Z$, 4.15 reads

$$
\partial_{Z} \psi+\frac{1}{2 \ell}\left(\frac{1}{B}+\frac{1}{\bar{B}}\right)=0 .
$$


Using the definition of $H$ we get

$$
\dot{H}+\partial_{Z} \ln \psi_{1}+\frac{1}{2 \ell}\left(\frac{1}{B}+\frac{1}{\bar{B}}\right)=0
$$

where a dot denotes a derivative with respect to $Z-w-\bar{w}$. We can thus conclude that $\partial_{Z} \ln \psi_{1}=\gamma / \ell$ for some constant $\gamma$, i. e. , $\psi_{1}(Z)=\psi_{1}^{(0)} e^{\gamma Z / \ell}$. By shifting $Z$ one can set $\psi_{1}^{(0)}=1$. Calling $\chi=\psi_{+} / \psi_{-}$, the only remaining nontrivial Killing spinor equations read

$$
\begin{aligned}
\partial_{Z} \chi-2\left(\frac{\dot{\rho}}{\rho}-\dot{H}\right) \chi+2 i \dot{\varphi}+\frac{1}{\ell}\left(\frac{1}{B}-\frac{1}{\bar{B}}\right) & =0 \\
\partial_{Z} \chi-\left(2 \frac{\dot{\rho}}{\rho}-\dot{H}+\frac{\gamma}{\ell}\right) \chi-2 i e^{-2 H} \dot{\varphi}-\frac{1}{2 \ell}\left(\frac{1}{B}-\frac{1}{\bar{B}}\right) & =0 \\
\partial \chi+2\left(\frac{\dot{\rho}}{\rho}-\dot{H}\right) \chi-2 i \dot{\varphi}-\frac{1}{\ell}\left(\frac{1}{B}-\frac{1}{\bar{B}}\right) & =0 \\
\bar{\partial} \chi+2 \frac{\dot{\rho}}{\rho} \chi-2 i \dot{\varphi} & =0 \\
\frac{1}{2 \ell}\left(\frac{1}{B}-\frac{1}{\bar{B}}\right) \chi+2\left(1+e^{-2 H}\right) \frac{\dot{\rho}}{\rho}-\dot{H}+\frac{\gamma}{\ell} & =0
\end{aligned}
$$

Summing the first and the third equation yields $\chi=\chi(Z-w-\bar{w})$, so that we are left with

$$
\begin{aligned}
\dot{\chi}-2\left(\frac{\dot{\rho}}{\rho}-\dot{H}\right) \chi+2 i \dot{\varphi}+\frac{1}{\ell}\left(\frac{1}{B}-\frac{1}{\bar{B}}\right) & =0, \\
\dot{\chi}-\left(2 \frac{\dot{\rho}}{\rho}-\dot{H}+\frac{\gamma}{\ell}\right) \chi-2 i e^{-2 H} \dot{\varphi}-\frac{1}{2 \ell}\left(\frac{1}{B}-\frac{1}{\bar{B}}\right) & =0, \\
-\dot{\chi}+2 \frac{\dot{\rho}}{\rho} \chi-2 i \dot{\varphi} & =0, \\
\frac{1}{2 \ell}\left(\frac{1}{B}-\frac{1}{\bar{B}}\right) \chi+2\left(1+e^{-2 H}\right) \frac{\dot{\rho}}{\rho}-\dot{H}+\frac{\gamma}{\ell} & =0 .
\end{aligned}
$$

Adding (D.4) and (D.6) one gets

$$
\dot{H} \chi+\frac{1}{2 \ell}\left(\frac{1}{B}-\frac{1}{\bar{B}}\right)=0,
$$

which means that $\chi$ is purely imaginary. From (D.2) and (D.7) we obtain then the function $B$,

$$
\frac{1}{\ell B}+\frac{\gamma}{\ell}+\dot{H}(1+\chi)=0
$$


Using this, the remaining Killing spinor equations reduce further to

$$
\begin{aligned}
{\left[\left(1+e^{2 H}\right) \frac{\chi}{\rho^{2}}\right]-\frac{\gamma}{\ell}\left(e^{2 H} \frac{\chi}{\rho^{2}}\right) } & =0, \\
\left(\frac{\chi}{\rho^{2}}\right)+2 i \frac{\dot{\varphi}}{\rho^{2}} & =0, \\
\dot{H}\left(1+\chi^{2}\right)-2 \frac{\dot{\rho}}{\rho}\left(1+e^{-2 H}\right) & =\frac{\gamma}{\ell} .
\end{aligned}
$$

Note that (D.9) automatically implies the integrability condition for the system (4.18), (4.19), which reduces to

$$
\partial_{Z} \sigma_{w}=\frac{1}{4 \psi_{1}}\left(\frac{\chi}{\rho^{2}}\right)^{\cdot}, \quad \partial \sigma_{\bar{w}}-\bar{\partial} \sigma_{w}=-\frac{1}{2 \psi_{1}}\left(e^{2 H} \frac{\chi}{\rho^{2}}\right) .
$$

Thus, also equation (4.23) is satisfied, whereas (4.22) reads

$$
\left(1+e^{-2 H}\right) 2 \ddot{H}+\dot{H}^{2}\left(1+3 \chi^{2}\right)=\frac{\gamma^{2}}{\ell^{2}} .
$$

From (D.8) we obtain the phase $\varphi$ and the modulus $\rho$ of $B$,

$$
\tan \varphi=i \frac{\dot{H} \chi}{\frac{\gamma}{\ell}+\dot{H}}, \quad \frac{1}{\ell^{2} \rho^{2}}=\left(\frac{\gamma}{\ell}+\dot{H}\right)^{2}-\dot{H}^{2} \chi^{2} .
$$

Plugging this into equation (D.10) yields

$$
2 \ddot{H} \dot{H} \chi\left(1-\chi^{2}\right)-\dot{H}^{2} \dot{\chi}\left(1+3 \chi^{2}\right)+\frac{\gamma^{2}}{\ell^{2}} \dot{\chi}=0
$$

Using (D.13), this can be rewritten as

$$
2 \ddot{H} \chi\left[\dot{H} \chi\left(1-\chi^{2}\right)+\left(1+e^{-2 H}\right) \dot{\chi}\right]=0
$$

so that either $\ddot{H}=0$ or $\dot{H} \chi\left(1-\chi^{2}\right)+\left(1+e^{-2 H}\right) \dot{\chi}=0$. It is straightforward to show that the first case leads to $\mathrm{AdS}_{4}$, whereas the second one implies

$$
\left(e^{2 H}+1\right) \frac{\chi^{2}}{1-\chi^{2}}=-\alpha^{2},
$$

where $\alpha$ is a real integration constant. Equations (D.9) and (D.11) are then identically satisfied. Solving (D.14) for $\chi$ and plugging into (D.13) yields finally the ordinary differential equation (4.50), which determines half-supersymmetric solutions with $G_{0}=$ 0. Putting together all our results, we obtain (4.52) for the metric. Note that in the 
case $\gamma \neq 0$ one can always set $\gamma=1$ by rescaling the coordinates.

The second Killing spinor for these backgrounds is given by

$$
\alpha^{T}=\left(\alpha_{0}, \rho^{-2} e^{-\gamma Z / \ell}, \frac{\chi+1}{2 \rho} e^{H}, \frac{\chi-1}{2 \rho} e^{H}\right),
$$

where

$$
\alpha_{0}=-\frac{2 \gamma t}{\ell}+\hat{\alpha}_{0}(Z, w, \bar{w})
$$

and $\hat{\alpha}_{0}$ is a solution of the system

$$
\begin{aligned}
\partial Z \hat{\alpha}_{0} & =\frac{1}{\psi_{1} \rho^{2}}\left[\frac{\dot{\rho}}{\rho}-i \dot{\varphi}+\frac{\gamma}{2 \ell}\right], \\
\partial \hat{\alpha}_{0} & =-\frac{2 \gamma}{\ell} \sigma_{w}+\frac{1}{\psi_{1} \rho^{2}}\left[-\frac{\dot{\rho}}{\rho}+i \dot{\varphi}\right], \\
\bar{\partial} \hat{\alpha}_{0} & =-\frac{2 \gamma}{\ell} \sigma_{\bar{w}}+\frac{1}{\psi_{1} \rho^{2}}\left[-\frac{\dot{\rho}}{\rho}+i \dot{\varphi}\right]+\frac{\gamma \chi e^{2 H}}{\ell \psi_{1} \rho^{2}} .
\end{aligned}
$$

It is straightforward to verify that the integrability conditions for this system are already implied by (D.9), (D.10) and (D.12).

Consider now the case $\psi_{-}=0$. From the difference of equations (4.38) and (4.41) it follows that $b^{\prime} / b$ is real. Then (4.38) and (4.44) imply that $\psi$ is a real function, depending only on $z, \psi_{1}=\psi_{1}(z)$. Moreover, since $\psi_{12}=\psi_{2}$, the difference of equations (4.45) and (4.46) imply that $b^{\prime} / b+1 / \ell b$ is imaginary.

The conditions $b^{\prime} / b$ real and $b^{\prime} / b+1 / \ell b$ imaginary can be satisfied simultaneously in three different ways:

- $b^{\prime} / b=0$ hence $b=b(w, \bar{w})$ is an imaginary function independent of $z$. This case is solved completely in section 5.3 .

- $b^{\prime} / b+1 / \ell b=0$ implies $b=-z / \ell+c$ and corresponds to $\mathrm{AdS}_{2} \times \mathbb{H}^{2}$, analyzed in section 5.1.1. It is also a subcase of the following, general case,

- if we are not in one of the previous special cases, the function $b$ must take the form

$$
b=-\frac{1}{\ell} \frac{z}{1-i Y(w, \bar{w})},
$$

where $Y(w, \bar{w})$ is some real function to be determined.

We thus have to solve just for the ansatz (D.16). Equation (4.38) implies $\psi_{1}^{\prime} / \psi_{1}=b^{\prime} / b$ than is solved by $\psi_{1}=z$, where we have reabsorbed the integrability constant in the 
scale of $z$. Equation (4.39) (or equivalently (4.43)) tells us that $\psi_{2}=\psi_{2}(w, \bar{w})$, so that the remaining independent equations read

$$
\begin{aligned}
i z^{2} e^{-2 \psi} \frac{\bar{\partial} Y}{1+Y^{2}}-\psi_{2} & =0 \\
\partial \psi_{2}+\partial\left[\log \left(1+Y^{2}\right)+2 \psi\right] \psi_{2}-i Y & =0 \\
\bar{\partial} \psi_{2}+\bar{\partial} \log \left(1+Y^{2}\right) \psi_{2} & =0
\end{aligned}
$$

The first equation allows us to define a function $H(w, \bar{w})$ such that

$$
e^{\psi}=z e^{H(w, \bar{w})}
$$

while the last one implies that there must exist a holomorphic function $C(w)$ such that

$$
\psi_{2}=\frac{C(w)}{1+Y^{2}}
$$

Thus we are left with

$$
\begin{aligned}
e^{2 H} C(w) & =i \bar{\partial} Y, \\
\partial\left[e^{2 H} C(w)\right] & =i e^{2 H} Y\left(1+Y^{2}\right) .
\end{aligned}
$$

This set of equations automatically implies the integrability condition for the system (4.18), (4.19), which reduces to

$$
\begin{aligned}
\partial_{z} \sigma & =i \frac{\ell^{2}}{2} \frac{\partial Y}{z^{2}} \\
\partial \bar{\sigma}-\bar{\partial} \sigma & =i \ell^{2} e^{2 H} Y\left(1+Y^{2}\right) \frac{1}{z} .
\end{aligned}
$$

Thus also (4.23), which reads

$$
\partial \bar{\partial} Y-e^{2 H} Y\left(1+Y^{2}\right)=0
$$

is satisfied and it turns out that also the Bianchi identity (4.22), namely

$$
\partial \bar{\partial} 2 H-e^{2 H}\left(1+3 Y^{2}\right)=0
$$

holds. We conclude that a solution to the system (D.19), (D.20) describes a 1/2-BPS configuration of the "gravitational Chern-Simons" system discussed in [16]. If $C(w)=0$ then necessarily also $Y=0$ so that we are left with AdS. If $C(w) \neq 0$ then we can define new variables $W$ and $\bar{W}$ such that

$$
\partial_{W}=C(w) \partial, \quad \partial_{\bar{W}}=\bar{C}(\bar{w}) \bar{\partial}
$$


so that we have

$$
\begin{aligned}
e^{2 H} C \bar{C} & =i \partial_{\bar{W}} Y, \\
\partial_{W}\left[e^{2 H} C \bar{C}\right] & =i e^{2 H} C \bar{C} Y\left(1+Y^{2}\right) .
\end{aligned}
$$

As what we did in the previous case, we can set $C(w)=1$ using the residual gauge invariance $w \mapsto W(w), \psi \mapsto \tilde{\psi}=\psi-\frac{1}{2} \ln (\mathrm{d} W / \mathrm{d} w)-\frac{1}{2} \ln (\mathrm{d} \bar{W} / \mathrm{d} \bar{w})$ leaving invariant the metric $e^{2 \psi} \mathrm{d} w \mathrm{~d} \bar{w}$. We can thus take $W=w$ without loss of generality, and get

$$
\begin{aligned}
e^{2 H} & =i \bar{\partial} Y, \\
2 \partial H & =i Y\left(1+Y^{2}\right) .
\end{aligned}
$$

(D.26) implies $Y=Y[i(w-\bar{w})]$ and hence $H=H[i(w-\bar{w})]$. Denoting with a dot the derivative with respect to the combination $i(w-\bar{w})$ we have

$$
\begin{aligned}
e^{2 H} & =\dot{Y}, \\
2 \dot{H} & =Y\left(1+Y^{2}\right) .
\end{aligned}
$$

The equations for the shift form can now be integrated, giving

$$
\sigma=\frac{\ell^{2}}{2 z} \dot{Y} \mathrm{~d}(w+\bar{w})
$$

Plugging (D.27) into (D.28) leads to

$$
\ddot{Y}=\dot{Y} Y\left(1+Y^{2}\right)
$$

which, integrated once, gives

$$
\dot{Y}=\frac{L}{\ell^{4}}-\frac{k}{2} Y^{2}+\frac{1}{4} Y^{4} \equiv P(Y),
$$

where $L$ is a real constant and $k=-1^{21}$. We can thus use $Y$ as a new coordinate, instead of $i(w-\bar{w})$. Call $X=w+\bar{w}$, so that the solution reads

$$
\begin{aligned}
\mathrm{d} s^{2} & =-\frac{4}{\ell^{2}} \frac{z^{2}}{1+Y^{2}}\left[\mathrm{~d} t+\frac{\ell^{2}}{2 z} P_{\mathcal{C}}(Y) \mathrm{d} X\right]^{2}+\frac{\ell^{2}}{4} \frac{1+Y^{2}}{z^{2}}\left[\mathrm{~d} z^{2}+z^{2}\left(P_{\mathcal{C}}(Y) \mathrm{d} X^{2}+\frac{\mathrm{d} Y^{2}}{P_{\mathcal{C}}(Y)}\right)\right] \\
\mathcal{A} & =\frac{2}{\ell} z \frac{Y}{1+Y^{2}} \mathrm{~d} t+\ell Y\left[\frac{P_{\mathcal{C}}(Y)}{1+Y^{2}}-\frac{1}{4}\left(1+Y^{2}\right)\right] \mathrm{d} X+\frac{\ell}{2} \frac{\mathrm{d} Y}{1+Y^{2}}
\end{aligned}
$$

\footnotetext{
${ }^{21}$ The link with the notation of [48], where $\mathcal{C}$ and $k$ are the Casimirs of the Poisson sigma model equivalent to the dimensionally reduced gravitational Chern-Simons model in $2 \mathrm{D}$, is given by $2 \mathcal{C}=$ $\mathcal{L} / \ell^{\triangle}$.
} 
We can thus finally compute the second Killing spinor, with the result

$$
\begin{aligned}
\epsilon_{2}= & -\left[\frac{\ell^{2}}{2 z}\left(1+Y^{2}\right)+2 t\right] 1-\frac{\ell^{2}}{z} \sqrt{P_{\mathcal{C}}(Y)} \sqrt{\frac{1+i Y}{1-i Y}} e^{1}+ \\
& +\left[\frac{\ell^{2}}{z}\left(1+Y^{2}\right)+\frac{\ell}{2}(1+i Y)+\frac{2}{\ell} \frac{z t}{1-i Y}\right] e^{2}+\ell \sqrt{\frac{P_{\mathcal{C}}(Y)}{1+Y^{2}}} e^{1} \wedge e^{2}
\end{aligned}
$$

\section{References}

[1] M. Berger, "Sur les groupes d'holonomie homogènes de variétés à connexion affine et des variétés riemanniennes," Bul. Soc. Math. France 83 (1955) 279.

[2] R. L. Bryant, "Pseudo-Riemannian metrics with parallel spinor fields and vanishing Ricci tensor," Sémin. Congr., 4 Soc. Math. France, Paris (2000) 53.

[3] J. M. Figueroa-O'Farrill, "Breaking the M-waves," Class. Quant. Grav. 17 (2000) 2925 [arXiv:hep-th/9904124].

[4] A. Batrachenko and W. Y. Wen, "Generalized holonomy of supergravities with 8 real supercharges," Nucl. Phys. B 690 (2004) 331 [arXiv:hep-th/0402141].

[5] K. P. Tod, "All metrics admitting supercovariantly constant spinors," Phys. Lett. B 121 (1983) 241.

[6] J. Kowalski-Glikman, "Positive Energy Theorem And Vacuum States For The Einstein-Maxwell System," Phys. Lett. B 150 (1985) 125.

[7] J. P. Gauntlett, D. Martelli, S. Pakis and D. Waldram, "G-structures and wrapped NS5-branes," Commun. Math. Phys. 247 (2004) 421 [arXiv:hep-th/0205050].

[8] J. P. Gauntlett, J. B. Gutowski, C. M. Hull, S. Pakis and H. S. Reall, "All supersymmetric solutions of minimal supergravity in five dimensions," Class. Quant. Grav. 20, 4587 (2003) [arXiv:hep-th/0209114].

[9] J. B. Gutowski, D. Martelli and H. S. Reall, "All supersymmetric solutions of minimal supergravity in six dimensions," Class. Quant. Grav. 20, 5049 (2003) [arXiv:hep-th/0306235].

[10] P. Meessen and T. Ortín, "The supersymmetric configurations of $\mathrm{N}=2, \mathrm{~d}=4$ supergravity coupled to vector supermultiplets," Nucl. Phys. B 749, 291 (2006) [arXiv:hep-th/0603099].

[11] J. P. Gauntlett and S. Pakis, "The geometry of D = 11 Killing spinors," JHEP 0304, 039 (2003) [arXiv:hep-th/0212008]. 
[12] J. P. Gauntlett and J. B. Gutowski, "All supersymmetric solutions of minimal gauged supergravity in five dimensions," Phys. Rev. D 68, 105009 (2003) [Erratum-ibid. D 70, 089901 (2004)] [arXiv:hep-th/0304064].

[13] M. M. Caldarelli and D. Klemm, "All supersymmetric solutions of $N=2, D=4$ gauged supergravity," JHEP 0309 (2003) 019 [arXiv:hep-th/0307022].

[14] J. P. Gauntlett, J. B. Gutowski and S. Pakis, "The geometry of D = 11 null Killing spinors," JHEP 0312, 049 (2003) [arXiv:hep-th/0311112].

[15] M. Cariglia and O. A. P. Mac Conamhna, "The general form of supersymmetric solutions of $\mathrm{N}=(1,0) \mathrm{U}(1)$ and $\mathrm{SU}(2)$ gauged supergravities in six dimensions," Class. Quant. Grav. 21 (2004) 3171 [arXiv:hep-th/0402055].

[16] S. L. Cacciatori, M. M. Caldarelli, D. Klemm and D. S. Mansi, "More on BPS solutions of $N=2, D=4$ gauged supergravity," JHEP 0407 (2004) 061 [arXiv:hep-th/0406238].

[17] M. Cariglia and O. A. P. Mac Conamhna, "Timelike Killing spinors in seven dimensions," Phys. Rev. D 70 (2004) 125009 [arXiv:hep-th/0407127].

[18] J. B. Gutowski and W. Sabra, "General supersymmetric solutions of five-dimensional supergravity," JHEP 0510, 039 (2005) [arXiv:hep-th/0505185].

[19] J. Bellorin and T. Ortín, "All the supersymmetric configurations of $\mathrm{N}=4, \mathrm{~d}=4$ supergravity," Nucl. Phys. B 726, 171 (2005) [arXiv:hep-th/0506056].

[20] M. Huebscher, P. Meessen and T. Ortín, "Supersymmetric solutions of $\mathrm{N}=2 \mathrm{~d}=4$ SUGRA: The whole ungauged shebang," Nucl. Phys. B 759, 228 (2006) [arXiv:hep-th/0606281].

[21] J. Bellorin, P. Meessen and T. Ortín, "All the supersymmetric solutions of $\mathrm{N}=1, \mathrm{~d}=$ 5 ungauged supergravity,” JHEP 0701, 020 (2007) [arXiv:hep-th/0610196].

[22] J. Gillard, U. Gran and G. Papadopoulos, "The spinorial geometry of supersymmetric backgrounds," Class. Quant. Grav. 22, 1033 (2005) [arXiv:hep-th/0410155].

[23] U. Gran, G. Papadopoulos and D. Roest, "Systematics of M-theory spinorial geometry," Class. Quant. Grav. 22, 2701 (2005) [arXiv:hep-th/0503046].

[24] U. Gran, J. Gutowski and G. Papadopoulos, "The spinorial geometry of supersymmetric IIB backgrounds," Class. Quant. Grav. 22, 2453 (2005) [arXiv:hep-th/0501177].

[25] U. Gran, J. Gutowski and G. Papadopoulos, "The G(2) spinorial geometry of supersymmetric IIB backgrounds," Class. Quant. Grav. 23, 143 (2006) [arXiv:hep-th/0505074]. 
[26] U. Gran, J. Gutowski, G. Papadopoulos and D. Roest, "Systematics of IIB spinorial geometry," Class. Quant. Grav. 23 (2006) 1617 [arXiv:hep-th/0507087].

[27] U. Gran, J. Gutowski, G. Papadopoulos and D. Roest, "N = 31 is not IIB," JHEP 0702, 044 (2007) [arXiv:hep-th/0606049].

[28] J. Grover, J. B. Gutowski and W. Sabra, "Vanishing preons in the fifth dimension," Class. Quant. Grav. 24, 417 (2007) [arXiv:hep-th/0608187].

[29] J. Grover, J. B. Gutowski and W. A. Sabra, "Maximally minimal preons in four dimensions," arXiv:hep-th/0610128.

[30] U. Gran, J. Gutowski, G. Papadopoulos and D. Roest, "N = 31, D = 11," JHEP 0702, 043 (2007) [arXiv:hep-th/0610331].

[31] M. M. Caldarelli and D. Klemm, "Supersymmetric Goedel-type universe in four dimensions," Class. Quant. Grav. 21 (2004) L17 [arXiv:hep-th/0310081].

[32] U. Gran, G. Papadopoulos, D. Roest and P. Sloane, "Geometry of all supersymmetric type I backgrounds," arXiv:hep-th/0703143.

[33] S. T. C. Siklos, "Lobatchevski plane gravitational waves," in: Galaxies, axisymmetric systems and relativity, ed. M. A. H. MacCallum, Cambridge University Press, Cambridge (1985).

[34] N. Alonso-Alberca, P. Meessen and T. Ortín, "Supersymmetry of topological Kerr-Newman-Taub-NUT-adS spacetimes," Class. Quant. Grav. 17 (2000) 2783 [arXiv:hep-th/0003071].

[35] S. Cacciatori, D. Klemm and D. Zanon, " $w_{\infty}$ algebras, conformal mechanics, and black holes," Class. Quant. Grav. 17 (2000) 1731 [arXiv:hep-th/9910065].

[36] M. M. Caldarelli and D. Klemm, "Supersymmetry of anti-de Sitter black holes," Nucl. Phys. B 545 (1999) 434 [arXiv:hep-th/9808097].

[37] R. Penrose, "Any space-time has a plane wave as a limit", in Differential geometry and relativity, Reidel, Dordrecht (1976) pp. 27175,

[38] R. Gueven, "Plane wave limits and T-duality," Phys. Lett. B 482 (2000) 255 [arXiv:hep-th/0005061].

[39] E. Witten, "Instability Of The Kaluza-Klein Vacuum," Nucl. Phys. B 195 (1982) 481.

[40] D. Birmingham and M. Rinaldi, "Bubbles in anti-de Sitter space," Phys. Lett. B 544, 316 (2002) [arXiv:hep-th/0205246]. 
[41] V. Balasubramanian and S. F. Ross, "The dual of nothing," Phys. Rev. D 66 (2002) 086002 [arXiv:hep-th/0205290].

[42] D. Astefanesei and G. C. Jones, "S-branes and (anti-)bubbles in (A)dS space," JHEP 0506 (2005) 037 [arXiv:hep-th/0502162].

[43] S. Deser, R. Jackiw and S. Templeton, "Topologically massive gauge theories," Annals Phys. 140 (1982) 372 [Erratum-ibid. 185 (1988 APNYA,281,409-449.2000) 406.1988 APNYA,281,409].

[44] G. Guralnik, A. Iorio, R. Jackiw and S. Y. Pi, "Dimensionally reduced gravitational Chern-Simons term and its kink," Annals Phys. 308 (2003) 222 [arXiv:hep-th/0305117].

[45] I. A. Bandos, J. A. de Azcárraga, J. M. Izquierdo and J. Lukierski, "BPS states in M-theory and twistorial constituents," Phys. Rev. Lett. 86 (2001) 4451 [arXiv:hep-th/0101113].

[46] I. A. Bandos, J. A. de Azcarraga and O. Varela, "On the absence of BPS preonic solutions in IIA and IIB supergravities," JHEP 0609 (2006) 009 [arXiv:hep-th/0607060].

[47] J. Figueroa-O'Farrill and S. Gadhia, "M-theory preons cannot arise by quotients," arXiv:hep-th/0702055.

[48] D. Grumiller and W. Kummer, "The classical solutions of the dimensionally reduced gravitational Chern-Simons theory," Annals Phys. 308, 211 (2003) [arXiv:hep-th/0306036].

[49] S. L. Cacciatori, M. M. Caldarelli, D. Klemm, D. S. Mansi, P. Meessen, T. Ortín and D. Roest, "All supersymmetric solutions of matter-coupled gauged $\mathcal{N}=2, D=4$ supergravity," in preparation.

[50] H. B. Lawson and M. L. Michelsohn, "Spin Geometry," Princeton, UK: Univ. Pr. (1998) 